Draft version August 16, 2018

Preprint typeset using $\mathrm{LAT}_{\mathrm{E}} \mathrm{X}$ style emulateapj v. 5/2/11

\title{
THE AKARI 2.5-5.0 MICRON SPECTRAL ATLAS OF TYPE-1 ACTIVE GALACTIC NUCLEI: BLACK HOLE MASS ESTIMATOR, LINE RATIO, AND HOT DUST TEMPERATURE
}

\author{
Dohyeong Kim ${ }^{1,2}$, Myungshin Im $^{1,2}$, Ji Hoon Kim ${ }^{1,2,3}$, Hyunsung David Jun ${ }^{1,2}$, Jong-Hak Woo ${ }^{2}$, Hyung Mok \\ Lee $^{2}$, Myung Gyoon Lee ${ }^{2}$, Takao Nakagawa ${ }^{4}$, Hideo Matsuhara ${ }^{4}$, Takehiko Wada ${ }^{4}$, Shinki Oyabu ${ }^{5}$, Toshinobu \\ Takagi $^{4}$, Youichi Ohyama ${ }^{6}$, AND Seong-Kook Lee ${ }^{1,2}$ \\ Draft version August 16, 2018
}

\begin{abstract}
We present $2.5-5.0 \mu \mathrm{m}$ spectra of 83 nearby $(0.002<z<0.48)$ and bright $(K<14 \mathrm{mag})$ type-1 active galactic nuclei (AGNs) taken with the Infrared Camera (IRC) on board AKARI. The 2.5$5.0 \mu \mathrm{m}$ spectral region contains emission lines such as $\operatorname{Br} \beta(2.63 \mu \mathrm{m}), \operatorname{Br} \alpha(4.05 \mu \mathrm{m})$, and polycyclic aromatic hydrocarbons (PAH; $3.3 \mu \mathrm{m})$, which can be used for studying the black hole (BH) masses and star formation activities in the host galaxies of AGNs. The spectral region also suffers less dust extinction than in the ultra violet (UV) or optical wavelengths, which may provide an unobscured view of dusty AGNs. Our sample is selected from bright quasar surveys of Palomar-Green (PG) and SNUQSO, and AGNs with reverberation-mapped BH masses from Peterson et al. (2004). Using 11 AGNs with reliable detection of Brackett lines, we derive the Brackett-line-based BH mass estimators. We also find that the observed Brackett line ratios can be explained with the commonly adopted physical conditions of the broad line region (BLR). Moreover, we fit the hot and warm dust components of the dust torus by adding photometric data of SDSS, 2MASS, WISE, and ISO to the AKARI spectra, finding hot and warm dust temperatures of $\sim 1100 \mathrm{~K}$ and $\sim 220 \mathrm{~K}$, respectively, rather than the commonly cited hot dust temperature of $1500 \mathrm{~K}$.

Subject headings: galaxies: active - galaxies: nuclei - quasars: emission lines - quasars: general infrared: galaxies
\end{abstract}

\section{INTRODUCTION}

An active galactic nucleus (AGN) is a bright central part of a galaxy that shines via the energy released by materials that falls toward the super massive black hole (SMBH). During its active phase, the AGN luminosity can outshine that of the host galaxy, emitting an enormous amount of energy $\left(10^{43}-10^{48} \mathrm{erg} \mathrm{s}^{-1}\right.$; e.g., Woo \& Urry 2002) over a wide range of wavelengths from gamma-ray to radio. The spectrum of an AGN provides us with important information concerning the structure and the physics around the SMBH that powers the AGN activity. High-energy photons, such as X-ray and UV, probe the accretion disk around the SMBH that gives off hot thermal emission. Optical photons trace the accretion disk or the broad and narrow line regions farther away from the SMBH. Meanwhile, infrared (IR) observations can trace the dust-heated radiation from the dusty torus or the star forming regions. Finally, jets can be probed in the radio.

\footnotetext{
dohyeong@astro.snu.ac.kr and mim@astro.snu.ac.kr

${ }^{1}$ Center for the Exploration of the Origin of the Universe (CEOU), Astronomy Program, Department of Physics and Astronomy, Seoul National University, Shillim-Dong, Kwanak-Gu Seoul 151-742, South Korea

2 Astronomy Program, Department of Physics and Astronomy, Seoul National University, Shillim-Dong, Kwanak-Gu, Seoul 151742, South Korea

3 Subaru Telescope, 650 North A'ohoku Place, Hilo, Hawaii 96720, U.S.A.

${ }^{4}$ Institute of Space and Astronautical Science, Japan Aerospace Exploration Agency, Sagamihara, Kanagawa 2525210, Japan

5 Graduate School of Science, Nagoya University, Furo-cho, Chikusa-ku, Nagoya, Aichi 464-8602, Japan

6 Institute of Astronomy and Astrophysics, Academia Sinica, P.O. Box 23-141, Taipei 106, Taiwan
}

In recent years, the near-infrared (NIR) wavelength region has become an important window for understanding AGNs. In general, hot dust emission is known to be the main contributor to the NIR light from an AGN (e.g., Barvainis 1987; Kobayashi et al. 1993), and many studies have been carried out using observations in the NIR to understand the dust torus structures, such as the covering factor (Mor et al. 2009; Mor \& Trakhtenbrot 2011), dust torus size (Minezaki et al. 2004; Suganuma et al. 2006), hot dust temperature $\left(T_{\mathrm{HD}} \sim 1500 \mathrm{~K}\right.$; e.g., Barvainis 1987; Kobavashi et al. 1993; Glikman et al. 2006; Landt et al. 2011), and the evolution of circumnuclear dust (Haas et al. 2003; Jiang et al. 2010; Hao et al. 2012; Jun \& Im 2013).

Additionally, the NIR wavelength region includes interesting emission/absorption line features such as the hydrogen Paschen and Brackett lines, the polycyclic aromatic hydrocarbon (PAH) emission feature at $3.3 \mu \mathrm{m}$, and the absorption lines from $\mathrm{H}_{2} \mathrm{O}$ ice $(3.1 \mu \mathrm{m}), \mathrm{CO}_{2}$ $(4.26 \mu \mathrm{m})$, and $\mathrm{CO}(4.67 \mu \mathrm{m})$ gas. These line features can be used to understand the BLR size, the BH mass $\left(M_{\mathrm{BH}}\right)$, the star formation, and the materials surrounding the nuclear region as outlined below.

Recently, NIR Paschen lines have been put forward as a useful $M_{\mathrm{BH}}$ estimator (Kim et al. 2010; Landt et al. 2011, 2013), since they are much less affected by dust extinction than the common $M_{\mathrm{BH}}$ estimators using UV/optical lines (Vestergaard \& Peterson 2006; McLure \& Dunlop 2004; Greene \& Ho 2005). A similar study can be extended to the Brackett lines which would be even less affected by the dust than the Paschen lines. In the case of $E(B-V)=2 \mathrm{mag}$ (a value corresponding to the typical color excess of dusty, red AGNs; 
e.g., Glikman et al. 2007; Urrutia et al. 2009), the $\operatorname{Br} \alpha$, $\operatorname{Br} \beta, \operatorname{P} \alpha$, and $\mathrm{P} \beta$ line fluxes would be suppressed by factors of $1.31,1.62,2.16$, and 3.97 , respectively, assuming the galactic extinction law with $R_{V}=3.07$ (McCall 2004). In other words, the $\operatorname{Br} \alpha$ line is three times less affected by the dust extinction than the $\mathrm{P} \beta$ line in this example.

The $3.3 \mu \mathrm{m}$ PAH feature has been suggested as a possible star formation indicator (Tokunaga et al. 1991), and has been detected in many local AGNs. A number of studies suggest that there may exist a correlation between the starburst (traced by the $3.3 \mu \mathrm{m}$ PAH feature) and the AGN activity (traced, e.g., by $5100 \AA$ continuum luminosity, hereafter, $\left.L_{5100}\right)$, although the correlation may break down at very high IR luminosities (Oi et al. 2010; Woo et al. 2012; Imanishi et al. 2011; Kim et al. 2012; Yamada et al. 2013). Finally, molecular lines such as $\mathrm{H}_{2} \mathrm{O}$ ice, $\mathrm{CO}_{2}$, and $\mathrm{CO}$ arise from molecular clouds or the diffused interstellar medium in the line of sight toward the AGN (Spoon et al. 2004), and they are useful tracers for the reservoir of molecular gas in the host galaxy and in the vicinity of an AGN. NIR hydrogen line ratios are also suggested as a useful means to understand the physical conditions of BLR (Ruff et al. 2012).

Studies of Brackett lines and PAH features have a special importance for understanding the evolutionary stages of galaxies, especially the dusty AGN phase. Dusty, red AGNs are AGNs whose optical light is obscured by the foreground dust (see Kim et al. 2010 for the demography of various types of red AGNs). They are suspected to be an intermediate population in the evolution of galaxies, situated between the dust enshrouded star forming phase (such as ultra luminous infrared galaxy, ULIRG) and the luminous AGN phase. During the red AGN stage, SMBHs are expected to grow rapidly providing feedback to the star formation of host galaxies (Hopkins et al. 2005; Li et al. 2007), but heavy dust extinction in host galaxies prohibits observational studies of the activities occurring in red, dusty AGNs. Brackett lines and PAH features can possibly serve as NIR spectral diagnostics that can explore the AGN and star formation activity of dusty, red AGNs due to the low extinction of the light in NIR.

While studies of NIR spectra at $\lambda<2.5 \mu \mathrm{m}$ are common, studies based on the $2.5-5.0 \mu \mathrm{m}$ spectra of nearby AGNs have been scarce, mainly due to the observational limitation caused either by atmospheric absorption or strong thermal background at $\lambda>2.5 \mu \mathrm{m}$ on the ground. In order to better understand the $2.5-5.0 \mu \mathrm{m}$ spectra of AGNs, we conducted a spectroscopic study of 83 nearby AGNs using the AKARI Infrared Camera (IRC) (Murakami et al. 2007; Onaka et al. 2007) as a part of the AKARI mission program, Quasar Spectroscopic Observation with NIR Grism (QSONG; Im 2010). The AKARI IRC covers the wavelength range $2.5-5.0 \mu \mathrm{m}$ with $\mathrm{R} \sim 120$ at $3.6 \mu \mathrm{m}$ and offers high sensitivity at these wavelengths thanks to the cold and transparent environment in space. The scientific goals of our study are to (1) provide a spectral atlas of nearby, bright AGNs that have been studied extensively in previous works; (2) establish the $M_{\mathrm{BH}}$ estimator using Brackett lines so that we can estimate $M_{\mathrm{BH}}$ reliably by reducing the dust extinction effect (the Brackett-based $M_{\mathrm{BH}}$ estimator will be an improvement over Paschen line-based estimators); (3) understand the correlation between the hot dust emission and other AGN properties; and (4) study the $3.3 \mu \mathrm{m}$ $\mathrm{PAH}$ feature in AGNs to understand the connection between AGN and star-formation activities. In this paper, we will describe the sample characteristics, present the 2.5-5.0 $\mu \mathrm{m} A K A R I$ spectra of 83 AGNs, derive the $M_{\mathrm{BH}}$ estimator using the hydrogen Brackett lines of 10 AGNs where the Brackett lines are well detected, and investigate the correlation between the hot dust emission and AGN properties. The investigation of the PAH feature will appear in a future work.

\section{THE SAMPLE AND OBSERVATION}

\subsection{The Sample}

When constructing the sample, we impose the following two criteria. In order to place one of the redshifted Brackett lines $(\operatorname{Br} \beta$ and $\operatorname{Br} \alpha)$ in the IRC spectroscopic wavelength range of $2.5-5.0 \mu \mathrm{m}$, our sample is limited to AGNs at $z<0.5$. In addition, we selected objects that are brighter than $14 \mathrm{mag}$ in the $K$ band to gain $\mathrm{S} / \mathrm{N}>$ 10 for the continuum detection.

Using the above criteria, we constructed a sample of 108 nearby, bright AGNs that are culled from the three different AGN source catalogs below. The first source of the sample is a catalog of 35 bright and nearby type- 1 AGNs with $M_{\mathrm{BH}}$ values measured from the reverberation mapping method (Peterson et al. 2004; Denney et al. 2010; Grier et al. 2008). The reverberation mapping method provides the reference $M_{\mathrm{BH}}$ values, which are used as the basis for deriving $M_{\mathrm{BH}}$ estimators using spectra taken at a single-epoch. Among the reverberation mapping sample, 31 AGNs were observed before $A K A R I$ stopped its operation in 2011. Note that two objects among these 31 AGNs, 3C 273 and NGC 7469, were observed through another $A K A R I$ mission program (AGNUL; PI: Takao Nakagawa). The AGNs that are selected from the reverberation mapping sample have a wide range of $M_{\mathrm{BH}}$ values, $10^{6.3}-10^{8.9} M_{\odot}$, and they are at $z=0.002-0.29$. The second source is the PG QSO catalog (Green et al. 1986), since PG QSOs are optically very bright and have been extensively studied in previous works (Kaspi et al. 2000; Vestergaard 2002; Vestergaard \& Peterson 2006). We find 69 PG QSOs at $z=0.03-0.48$ to satisfy our selection criteria, excluding those that are already included in the reverberation mapping sample. Among them, 48 were observed by $A K A R I$. For their $M_{\mathrm{BH}}$ values, we use the values derived from the $\mathrm{H} \beta$ line as listed in Vestergaard \& Peterson (2006) where the $M_{\mathrm{BH}}$ values span the range of $10^{6.5}-10^{9.7} M_{\odot}$. Finally, we also supplement the sample by four nearby bright quasars from Seoul National University Quasar Survey in Optical (SNUQSO; Im et al. 2007; Lee et al. 2008). SNUQSO is a survey of bright quasars missed by previous quasar survey at high and low galactic latitude (b $<\left|20^{\circ}\right|$ ), and it provides additional bright QSOs to the sample. In total, 108 bright, low-redshift QSOs are selected, of which 83 were observed by $A K A R I$

Figures 1 and 2 show the basic characteristics of the sample. AGNs in our sample are located at a redshift range of $z=0.002-0.48$, and span over a wide range of $M_{\mathrm{BH}}\left(10^{6.3}-10^{9.7} M_{\odot}\right)$, bolometric luminosity $\left(L_{\mathrm{bol}} ; 10^{43.1}-10^{47.2} \mathrm{erg} \mathrm{s}^{-1}\right)$ and accretion rate $(-2.35 \leq$ 


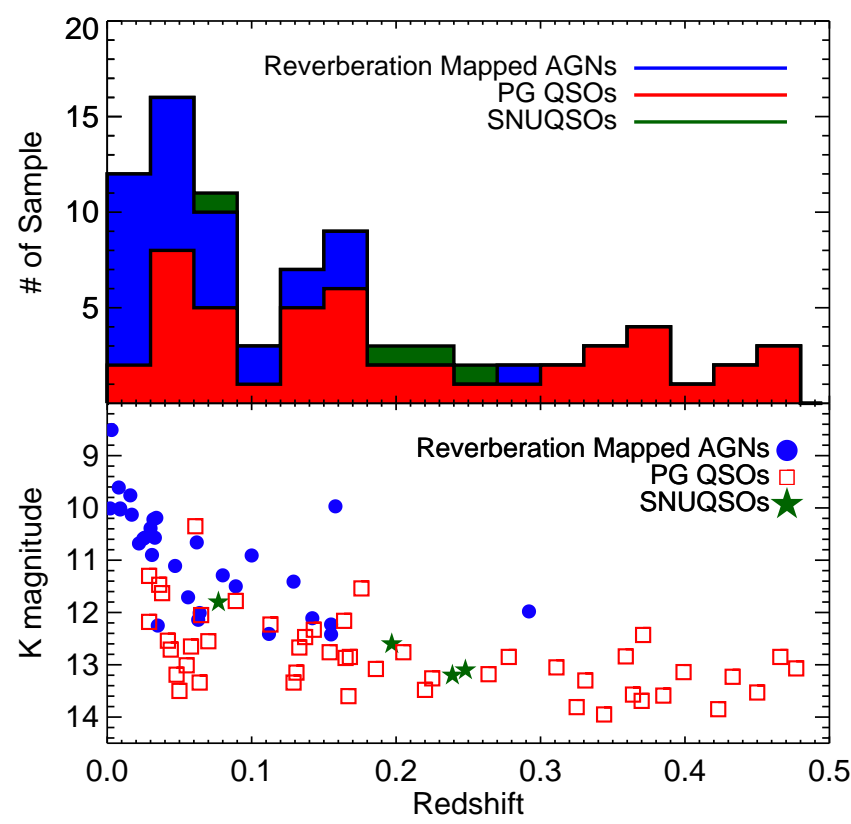

Figure 1. Top: redshift distribution of AGNs in our sample. The red, blue, and green histograms are for PG QSOs, reverberation mapped AGNs, and SNUQSOs, respectively. Bottom: redshift vs. $K$-band magnitude of our AGNs where the meaning of the colors is identical to the top panel. The $K$-band magnitudes include the host galaxy light. Note that PG QSOs are about two magnitude fainter than the reverberation mapped AGNs in apparent magnitude since they are in general at higher redshifts.

$\log ($ Eddington ratio $) \leq 0.43)$. The $L_{\mathrm{bol}}$ values of the $\mathrm{PG}$ QSOs and reverberation-mapped AGNs are estimated from $L_{5100}$ using a bolometric correction factor of 10.3 (Richards et al. 2006), and their $L_{5100}$ values are taken from Bentz et al. (2009); Vestergaard \& Peterson (2006). For SNUQSOs, the $\mathrm{H} \alpha$ luminosity from Im et al. (2007); Lee et al. $(2008)$ is converted to $L_{5100}$ using Equation (1) of Greene \& Ho (2005), and the converted $L_{5100}$ is translated to $L_{\mathrm{bol}}$ with a correction factor of 10.3. All of the objects observed by $A K A R I$ in our sample and their basic properties are presented in Table 1 . We note that the $M_{\mathrm{BH}}$ values in Table 1 are calculated with a recently updated virial factor of 5.1 (Woo et al. 2013).

\subsection{The Observation}

The 2.5-5.0 $\mu \mathrm{m}$ NIR spectra were obtained with the IRC infrared spectrograph (Onaka et al. 2007) on the $A K A R I$ satellite during phase 3 of the AKARI operation (Murakami et al. 2007), i.e., our observation was carried out during 2008-2011 after the helium cooling was over. We used the NG grism mode which provides a spectral resolution of $\mathrm{R}=120 \lambda / 3.6 \mu \mathrm{m}$. The spectral resolution corresponds to the FWHM velocity resolution of $2500 \mathrm{~km} \mathrm{~s}^{-1}$ or $\sigma=1062 \mathrm{~km} \mathrm{~s}^{-1}$ at $3.6 \mu \mathrm{m}$ which is adequate for studying the broad emission lines of quasars and AGNs whose typical FWHM velocity widths are greater than $2000 \mathrm{~km} \mathrm{~s}^{-1}$. Also, the wavelength coverage of 2.5-5.0 $\mu \mathrm{m}$ enables us to sample $\operatorname{Br} \beta, \operatorname{Br} \alpha$, and $\mathrm{PAH}$ at low redshift.

For most of the targets, three-pointing observation was performed where one-pointing observation is made of seven to nine frames with $44 \mathrm{~s}$ of exposure each. For a few sources, 1, 2, or 5 pointings were assigned (total integration time of 396 to 1848 seconds) due to the observational constraints of the telescope. Each target was placed at the center of a square-shaped slit window (1.0 $\times 1$ '. 0 size) designed to avoid confusing the spectrum with neighboring objects. Table 1 summarizes the observation of each target.

\subsection{Data Reduction}

The data reduction was performed using the standard data reduction software package "IRC Spectroscopy Toolkit for Phase 3 data Version 20110301 (2011/03/01)" (Ohyama et al. 2007), but with an additional procedure for cosmic ray/hot pixel removal to improve quality of the final spectrum. First, we used the standard IRC pipeline on each pointing data separately, up to the point before the extraction of the one-dimensional spectrum. Then, we removed hot or bad pixels in the stacked twodimensional spectrum using L.A.Cosmic van Dokkum 2001) in spectroscopic mode, since the AKARI phase 3 images typically come with many hot pixels and we wanted to remove effectively the hot pixels. We set the hot or bad pixel detection limit to $4.5 \sigma$ for removal of the hot pixels. Then, a one-dimensional spectrum of each pointing for each object was extracted using an extraction aperture width of 5 pixels, for which aperture correction is performed using the pipeline and assuming a point source PSF to correct for the missing flux due to the adoption of the finite aperture size. The extraction aperture width gives the optimal $\mathrm{S} / \mathrm{N}$ since the FWHM of PSFs in the IRC data is about 5 pixels $\left(7^{\prime \prime} \cdot 3\right)$. Note that the AGN spectrum can be contaminated by the host galaxy light, since the square-shaped slit window (1.0) does not completely block the light from the extended regions of a host galaxy. Hence, we are effectively sampling the light from the AGN+host galaxy within an observational rectangular aperture of $7^{\prime \prime} \cdot 3 \times$ 1.0 , which corresponds to physical scales of $1.49 \mathrm{kpc} \times$ $12.3 \mathrm{kpc}$ at $z=0.01$ and $32.5 \mathrm{kpc} \times 267 \mathrm{kpc}$ at $z=0.3$ where the long axis runs along the dispersion direction. In both cases, the aperture contains a significant portion of host galaxies, although we expect that the measured Brackett lines are dominated by emission from the nuclear region, as we describe in Section 4.1. Finally, the extracted one-dimensional spectra from all of the pointings of a source were combined. In this process, any remaining outlier pixels (hot or bad pixel features) in the one-dimensional spectrum were removed by $\sigma$ clipping with $4.5 \sigma$ where the median value and the $\sigma$ of a data point were determined including two adjacent data points. The wavelength and flux calibration was performed in the IRC pipeline and the expected accuracies of the wavelengths and fluxes are about $0.01 \mu \mathrm{m}$ and $10 \%$ (Ohyama et al. 2007), respectively.

To demonstrate the accuracy of the photometric calibration, we compare the fluxes of PG QSOs from the AKARI spectra to WISE $W 1$ and Spitzer IRAC Ch1 magnitudes. To simplify the comparison, we restrict the sample for the comparison to point-like sources (i.e., unresolved) in all of the AKARI, WISE, and Spitzer images. Thus, we restrict the sample for the photometry comparison to those in the PG QSO sample. For this comparison, we used the WISE $W 1$ magnitudes measured with a profile-fitting method provided by the WISE all-sky source catalog. However, for the 

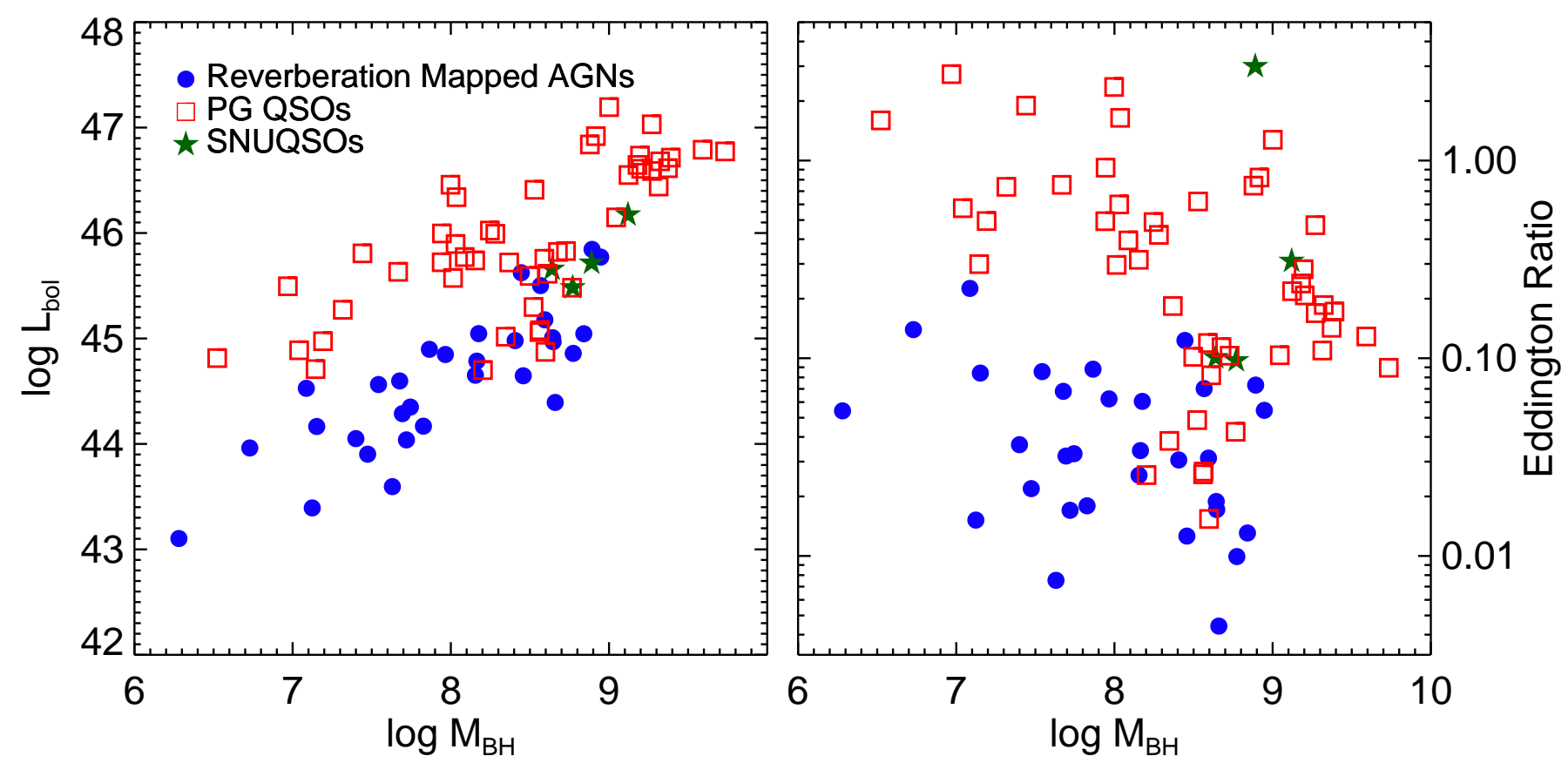

Figure 2. Left: $M_{\mathrm{BH}}$ values vs. $L_{\mathrm{bol}}$ values of AGNs in our sample. The meaning of the symbols are identical to Figure 1 . Our sample covers a wide range in $M_{\mathrm{BH}}\left(10^{6.3}-10^{9.7} M_{\odot}\right)$ and $L_{\mathrm{bol}}\left(10^{43.1}-10^{47.2} \mathrm{erg} \mathrm{s}{ }^{-1}\right)$. Right: $M_{\mathrm{BH}}$ values vs. Eddington ratios of our AGNs. Our AGN sample covers an extensive range of $\mathrm{BH}$ accretion rates $(-2.35 \leq \log$ (Eddington ratio) $\leq 0.43)$. Note that PG QSOs and SNUQSO AGNs are intrinsically more luminous and have higher accretion rates than the reverberation mapped AGNs.
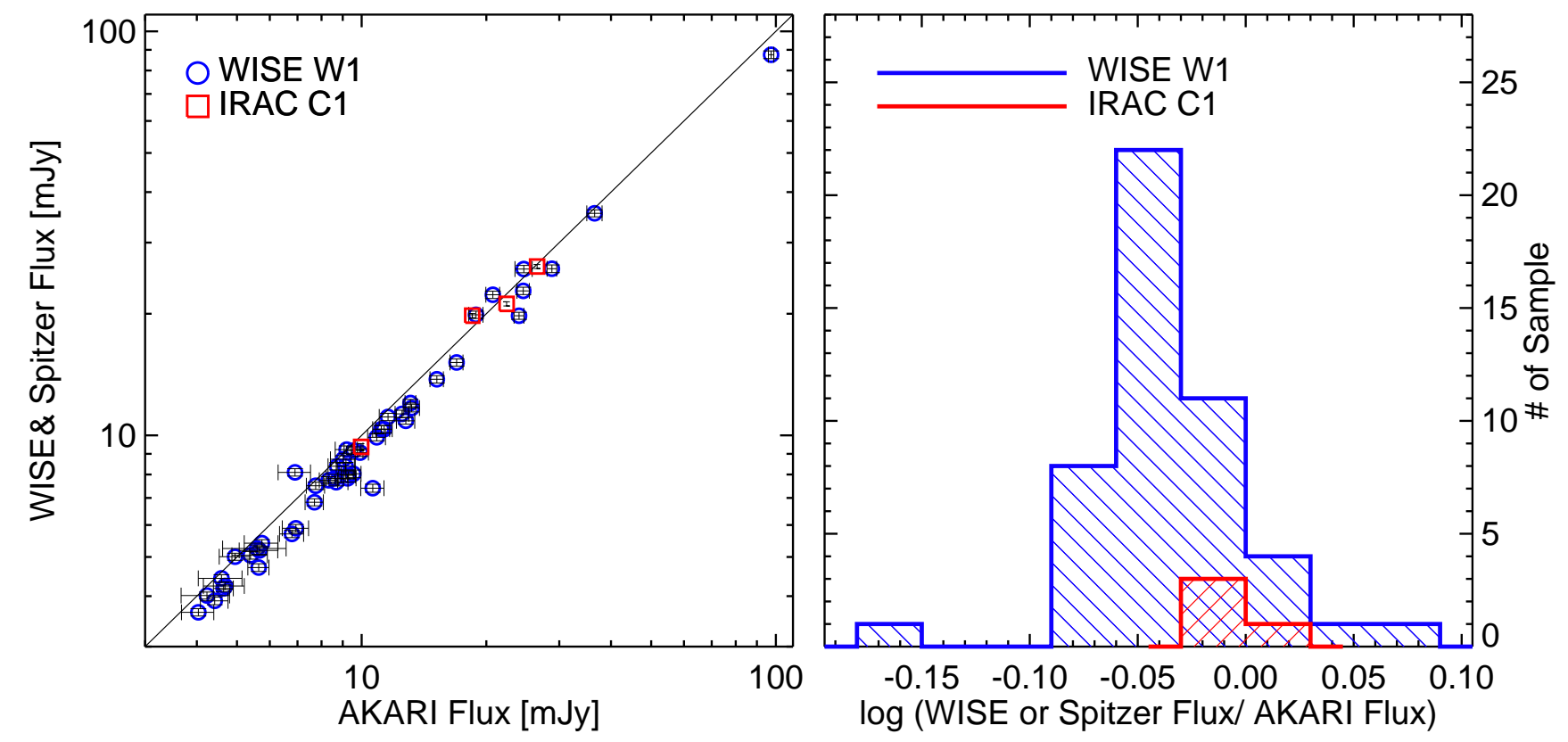

Figure 3. Left: comparison of the WISE $W 1$ or Spitzer IRAC $C h 1$ magnitudes vs. the WISE W 1 and Spitzer IRAC Ch 1 magnitudes derived from the AKARI spectra. The blue circles and the red squares indicate the WISE W1 and the Spitzer IRAC Ch1 fluxes, respectively. The black solid line is for the case where the AKARI, and WISE or Spitzer fluxes are identical. Right: Distribution of the ratios of the WISE or Spitzer fluxes to the AKARI fluxes of PG QSOs. The blue and the red histograms indicate the ratios for WISE and the Spitzer fluxes, respectively. 
Spitzer IRAC Ch1 magnitudes, we measured the magnitudes using IRAC $C h 1 \mathrm{PBCD}$ imaging data that we obtained from the Spitzer archive. In order to measure the magnitudes from the IRAC $C h 1$ PBCD imaging data, we used the IRAF daophot task with an aperture radius of $3 \times \mathrm{FWHM}$. Among the PG QSOs, five objects (PG 0007+106, PG 0050+124, PG 1100+772, PG 1501+106, and PG 1704+608) have IRAC Ch1 PBCD imaging data. Excluding PG 0050+124 which is too bright and saturated at the center of the IRAC image, we used four objects from the Spitzer archive for the comparison. Finally, using $A K A R I$ spectra, we calculated the corresponding WISE $W 1$ and Spitzer Ch1 magnitudes by applying appropriate filter transmission curves. Figure 3 shows the comparison of the fluxes from $A K A R I$ with those from WISE and Spitzer. The AKARI fluxes are on average $10 \%$ larger than the WISE fluxes, while the Spitzer Ch1 fluxes are in good agreement with the $A K A R I$ flux values. Although the reason for the systematic offset in the flux is not clear, we find the $10 \%$ difference to be acceptable considering the general flux calibration uncertainty of $A K A R I$ (Ohyama et al.|2007).

The flux errors of the spectra from the pipeline appear somewhat larger than the fluctuation of the data points (e.g., Figure 7). To check the flux error values from the pipeline, we used independent flux measurements derived from several pointing observations (Section 2.2), and we checked the determined uncertainty of the flux at each wavelength by adopting the rms value of the flux measurements from each pointing data. We find that the error derived this way agrees with the $A K A R I$ pipeline result, suggesting that the $A K A R I$ pipeline error measurement reflects unknown fluctuations in the continuum level determination or sensitivity fluctuations of IRC.

\subsection{Construction of Composite Spectrum}

In an attempt to increase the $\mathrm{S} / \mathrm{Ns}$ of the AGNs without Brackett line detections, we created a composite spectrum of the $48 \mathrm{PG}$ QSOs. The PG QSOs are less affected by host galaxy contamination than the reverberation-mapped sample due to bright luminosity ( $L_{\text {bol }}: 10^{44.6}-10^{47.2} \mathrm{erg} \mathrm{s}^{-1}$; Figure 2).

To construct the composite spectrum, we stacked, deredshifted, and flux-calibrated spectra of PG QSOs in logarithmic scale. Considering PG QSOs have different luminosity ranges, a geometric mean was used to construct the composite spectrum. We built a wavelength grid with a pixel resolution twice the spectral resolution of IRC $(\mathrm{R}=120 \lambda / 3.6 \mu \mathrm{m})$. In each wavelength grid, the weighted mean flux of each object was stacked for the geometric mean flux.

Figure 4 shows the composite spectrum of PG QSOs in $\lambda$ versus $F_{\nu}$. The location of key lines are also displayed in the figure. The composite spectrum is available in the supplemental material accompanying the article. Although we could not detect Brackett lines in individual PG QSO spectra, some of the NIR Hydrogen emission lines are now visible in the composite spectrum.

\section{AKARI NIR SPECTRA}

We show the $A K A R I$ spectra from our program in Figure 5. The machine-readable form of the spectra in Figure 5 are available. Several interesting lines are also indicated in Figure 5, such as the Hydrogen Brackett lines,

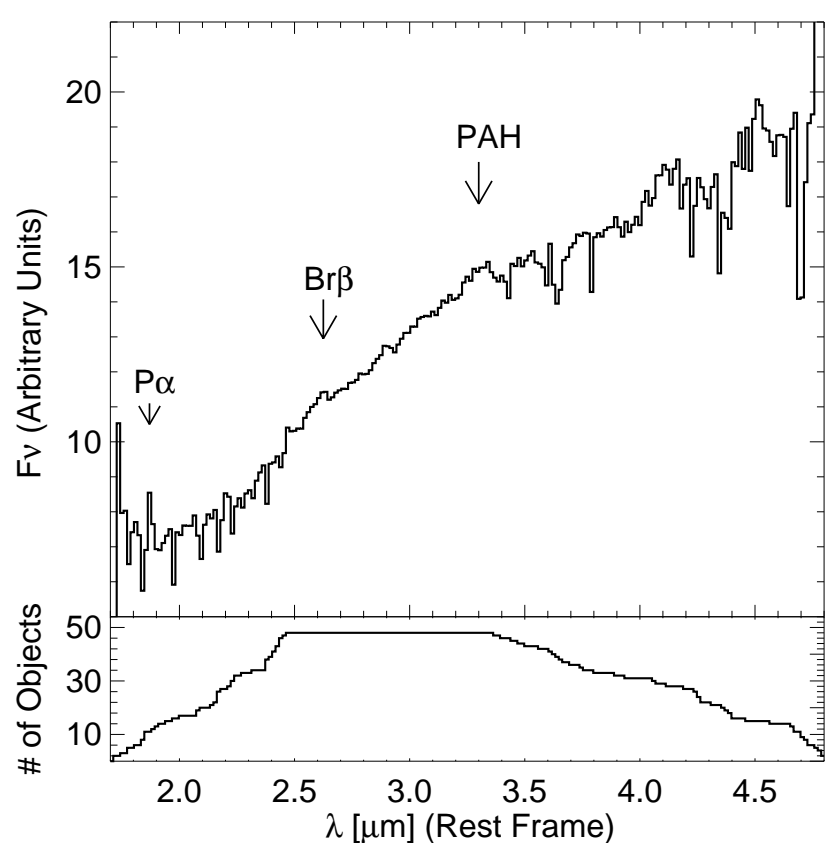

Figure 4. Top: composite spectrum of the 48 PG QSOs in arbitrary $F_{\nu}$ units. The composite spectrum indicates $\mathrm{P} \alpha, \operatorname{Br} \beta$, and PAH lines. Bottom: the number of spectra contributing for the composite spectrum.

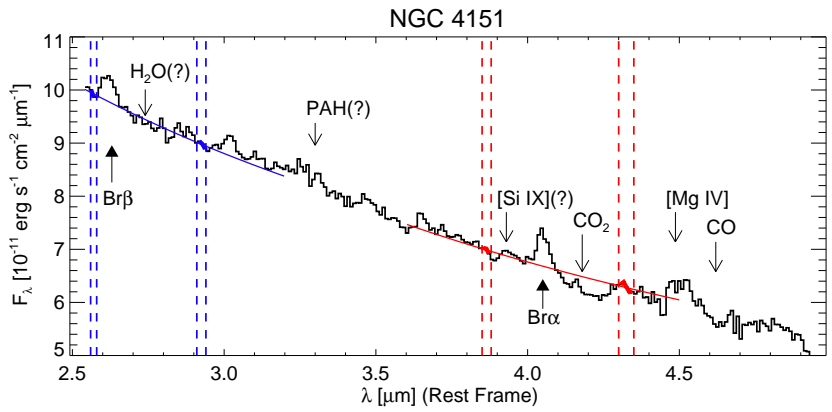

Figure 6. NIR spectrum $(2.5-5.0 \mu \mathrm{m})$ of NGC 4151. The 2.5$5.0 \mu \mathrm{m}$ wavelength window includes $\operatorname{Br} \alpha$ and $\operatorname{Br} \beta$ lines and various molecular lines such as $\mathrm{H}_{2} \mathrm{O}$, [Si IX], $\mathrm{CO}_{2},[\mathrm{Mg} \mathrm{IV}]$, and $\mathrm{CO}$. The blue and the red solid lines indicate the continuum fit to the regions around the $\operatorname{Br} \beta$ and $\operatorname{Br} \alpha$ lines, respectively. The dashed lines indicate the wavelength range used for the continuum fitting.

$3.3 \mu \mathrm{m} \mathrm{PAH}$, and other molecular/atomic lines. Br $\alpha$ and $\operatorname{Br} \beta$ lines are identified in 9 and 7 objects at $S / N>3$, respectively, and we mark the location of these lines in Figure 5 when they are identified. Potential detections are marked with a "?" sign. On the other hand, the Paschen lines are identified in 5 objects. All of the Brackett line detections come from the reverberation mapping sample whose members are brighter than the QSOs in the other two samples. The rather low detection rate of the Brackett lines reflect the reduced sensitivity and the increased number of detector defects during the warm mission phase of $A K A R I$.

\section{BRACKETT LINES}

Using the spectra we presented in the previous section, we measured the line fluxes and widths of Brackett lines and used them to estimate the $M_{\mathrm{BH}}$ values and the 
physical conditions of the gas in BLR. In this section, we will describe the measurements of the line fluxes and widths, present the Brackett-line based $M_{\mathrm{BH}}$ estimators, and discuss the BLR physical condition inferred from the line luminosity ratios of the NIR Hydrogen lines.

\subsection{Brackett Line Luminosity and Width}

In this subsection, we describe how the Brackett lines are fitted to derive line fluxes and widths. The fitting of the lines starts with the identification of the line in the deredshifted spectra. As mentioned already in the previous section, we find $\operatorname{Br} \alpha$ and $\operatorname{Br} \beta$ at $S / N>3$ in 9 and 7 AGNs, respectively. After the line identification, the continuum around the Brackett lines were determined by fitting a linear function to both sides of each Brackett line. Although a NIR spectrum of a quasar is dominated by the black body radiation from the hot part of the dust torus, the linear fit of the continuum should provide a reasonable approximation of the local continuum around each emission line (Landt et al. 2008; Kim et al. 2010). However, determining the best wavelength region for the continuum is not a simple task, because many molecular absorption or atomic emission lines exist around the Brackett lines. Such lines are $\mathrm{H}_{2} \mathrm{O}(2.53,2.66,2.73$, $2.75-3.55 \mu \mathrm{m})$, [Si IX] $(3.93 \mu \mathrm{m}), \mathrm{CO}_{2}(4.26 \mu \mathrm{m})$ and $\mathrm{CO}$ $(4.67 \mu \mathrm{m})$. Hence, we chose the continuum regions that are least affected by such lines. The wavelength regions adopted for the continuum determinations are listed in Table 2.

After continuum subtraction, we fit the Brackett lines and molecular/atomic lines simultaneously. Fitting of the molecular/atomic lines near the Brackett lines is necessary, since the fitting of the Brackett lines can be influenced by the presence of molecular/atomic lines such as the $\mathrm{H}_{2} \mathrm{O}$ absorption lines at 2.53 and $2.66 \mu \mathrm{m}$ (for $\operatorname{Br} \beta$ ), and the [Si IX] and $\mathrm{CO}_{2}$ gas line at 3.93 and $4.1-$ $4.4 \mu \mathrm{m}$ (for $\operatorname{Br} \alpha$ ). Figure 6 shows a $2.5-5.0 \mu \mathrm{m}$ spectrum of NGC 4151, which includes $\mathrm{Br} \alpha, \mathrm{Br} \beta, \mathrm{H}_{2} \mathrm{O}$, [Si IX], and $\mathrm{CO}_{2}$ lines. The Blue and red solid lines are the continuum levels used for the $\operatorname{Br} \beta$ and $\operatorname{Br} \alpha$ emission line measurements. The region bracketed by the dashed lines indicates the wavelength range used for the continuum fitting of each Brackett line.

We used a single Gaussian function to fit each line considering the low spectral resolution of the $A K A R I$ spectroscopy, setting the central wavelength, the line width, and the line flux to be free parameters. An interactive data language (IDL) procedure, MPFITEXPR (Markwardt 2009), was used to fit the lines. The fit provides the lineof-sight velocity dispersion, $\sigma$, and fluxes of the Brackett lines. Note that the narrow emission line does not significantly affect the line flux and $\sigma$ measurements. As a test, we set a model spectrum with a broad $(\sigma: 850$ $\left.-2500 \mathrm{~km} \mathrm{~s}^{-1}\right)$ and a narrow line component $(\sigma: 170$ $\mathrm{km} \mathrm{s}^{-1}$ ) at the $A K A R I$ spectral resolution. We adopt the flux ratios of the narrow to broad component flux to be 0.115 and 0.227 for $\operatorname{Br} \beta$ and $\operatorname{Br} \alpha$, respectively. These ratios are derived by first taking a median $\mathrm{H} \alpha$ narrow/broad component flux ratio in Landt et al. (2008) for five objects (Mrk 110, Mrk 79, NGC 7469, NGC 4593, and NGC 4151), and then converting the $\mathrm{H} \alpha$ flux to the Brackett flux based on the computation from the CLOUDY code (version 10.00; Ferland et al. 1998) for the BLR condition $\left(\alpha=-1.0, n=10^{9} \mathrm{~cm}^{-3}\right.$, and $U=$ $10^{-1.5}$; Kim et al. 2010) and the narrow line condition ( $n=10^{4.5} \mathrm{~cm}^{-3}$ and $\left.U=10^{-2} ; \operatorname{Cox} 2000\right)$. We fitted the degraded model emission line with a single Gaussian function. The measured flux and $\sigma$ values are 108\% (104 $-115 \%)$ and $95 \%(90-98 \%)$ compared to the original broad component properties, which would affect $M_{\mathrm{BH}}$ by a small amount, $\sim 0.03$ dex. It is also confirmed that the narrow emission lines in the NIR do not affect the line flux and $\sigma$ measurements as much $(<10 \%)$ in a relatively low-resolution spectra (e.g., Kim et al. 2010).

For further analysis in the following subsections, we exclude objects with substantial $\sigma$ errors $(>60 \%$ of the measured $\sigma$ value) or measured Brackett line FWHM $(2.35 \sigma)$ less than the spectral resolution $(\mathrm{R}=120 \lambda / 3.6 \mu \mathrm{m})$. The excluded cases are Mrk $110(\operatorname{Br} \beta)$ and Ark 120 $(\operatorname{Br} \alpha)$. Finally, eight $\operatorname{Br} \alpha$ and seven $\operatorname{Br} \beta$ lines are selected and used for further investigation of the line properties. All of these are from the reverberation mapping sample, since the PG QSOs are generally too faint for a reliable fitting of the lines (e.g., Figure 1).

Note that the selected Brackett lines show a small systematic shift in the wavelength of $\delta \lambda=\left(\lambda_{\text {measured }}-\lambda_{\text {lab }}\right)$ $\sim-0.014 \mu \mathrm{m}\left(\left\langle\delta \lambda_{\operatorname{Br} \alpha}\right\rangle=-0.015 \mu \mathrm{m}\right.$ and $\left\langle\delta \lambda_{\operatorname{Br} \beta}\right\rangle=$ $-0.013 \mu \mathrm{m})$. The $\operatorname{Br} \alpha$ and $\operatorname{Br} \beta$ lines are shifted by a similar amount in wavelength $\left(\delta \lambda_{\mathrm{Br} \alpha}-\delta \lambda_{\mathrm{Br} \beta}=-0.001 \pm\right.$ $0.005)$, suggesting that the wavelength shift applies to the entire spectral range. The shift is a bit larger than the expected wavelength offset error in the $A K A R I$ IRC of $0.01 \mu \mathrm{m}$ (Ohyama et al. 2007). The origin of the systematic offset is unclear, although it seems to be instrumental. A potential source of the offset is molecular/atomic absorption affecting the peak wavelength of the emission lines. However, if this were true, then we would expect a stronger offset in $\operatorname{Br} \alpha$ than in $\operatorname{Br} \beta$ since $\operatorname{Br} \beta$ is generally much less affected by molecular absorption features than $\operatorname{Br} \alpha$, but this was not the case.

The formal fitting errors are found to be about $27 \%$ (between $7 \%$ and $49 \%$ ) in $\sigma$ and $28 \%$ (between $6 \%$ and $46 \%$ ) in flux, due to low $\mathrm{S} / \mathrm{N}$ and the necessity of simultaneously fitting the other lines in the vicinity. Another source of error comes from the determination of the continuum. We varied the wavelength regions for the continuum fit from the values noted in Table 2 to see how strongly the changes in the continuum level affect the line fitting results. We find that the variation in the continuum level changes the derived $\sigma$ and flux values by $13 \%$ and $8 \%$ respectively. Overall, combining these two errors, we obtain the error in $\sigma$ and flux to be $33 \%$ and $30 \%$, which corresponds to 0.30 dex in $M_{\mathrm{BH}}$.

Energy sources of the Brackett lines could be $\mathrm{BH}$ activity or star formation activity. Considering the large slit aperture of the IRC and the extraction width we adopted, the measured Brackett line fluxes may include the star formation contribution from the host galaxy. To determine the dominant energy source of the Brackett lines, we compared the expected $L_{\mathrm{Br} \alpha}$ from star formation to the measured $L_{\mathrm{Br} \alpha}$. The relationships $L_{3.3 \mathrm{PAH}} / L_{\mathrm{IR}} \sim 10^{-3}$ (Mouri et al. 1990; Imanishi 2002) and $L_{\mathrm{Br} \alpha} / L_{\mathrm{IR}} \sim 10^{-4}$ (Imanishi et al. 2010) are expected for starburst galaxies, suggesting that the luminosity of the $\operatorname{Br} \alpha$ line from star formation is $10 \%$ of the $3.3 \mu \mathrm{m}$ PAH line luminosity. Among our seven $\operatorname{Br} \alpha$ - 

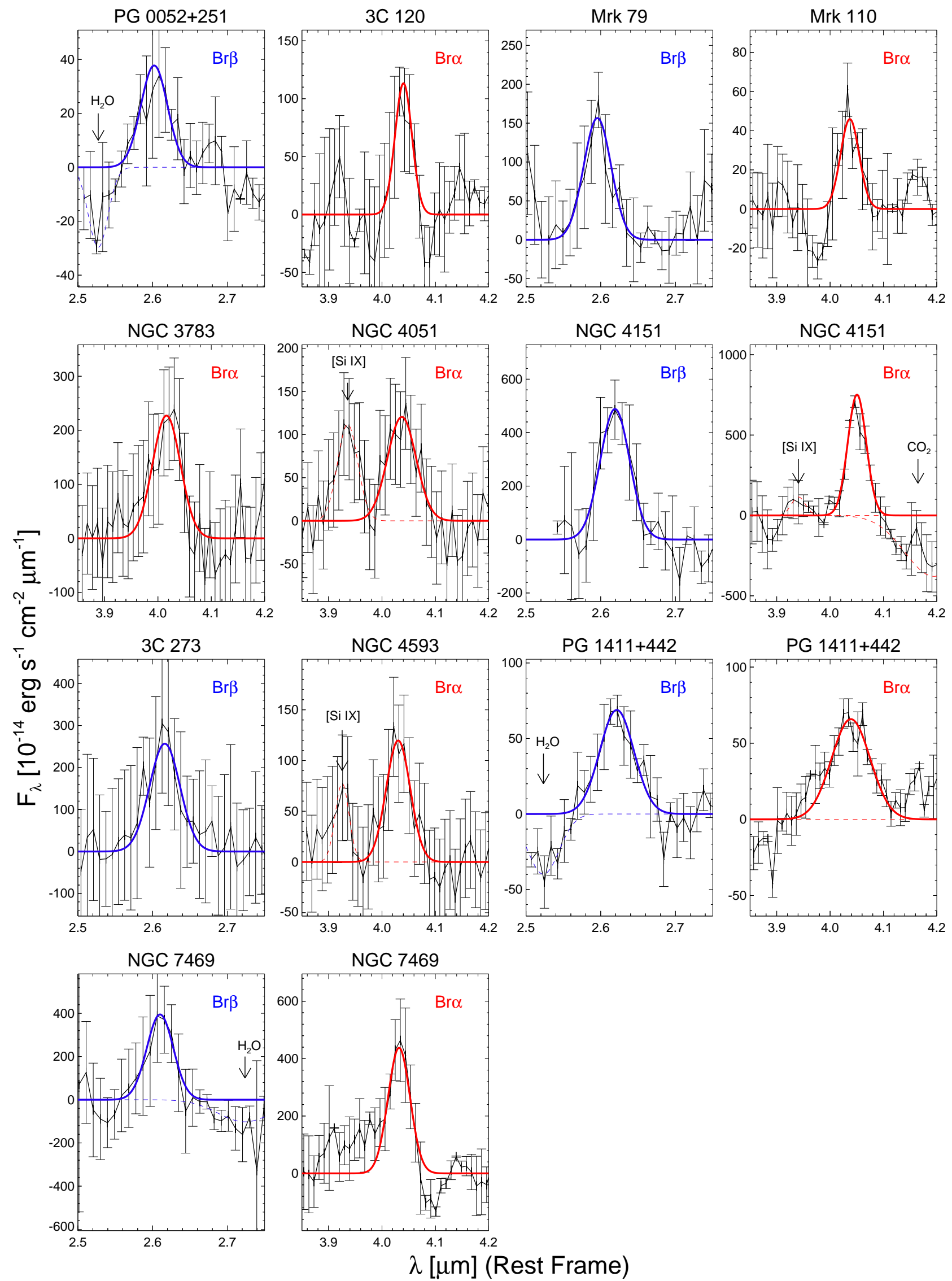

Figure 7. Gaussian fits of the Brackett lines of our sample. The black solid lines and bars indicate the observed spectra in rest frame and the associated errors. The blue and red lines indicate the fits for $\operatorname{Br} \beta$ and $\operatorname{Br} \alpha$, respectively. The dashed lines show the fits to the molecular/atomic lines around Brackett lines, such as $\mathrm{H}_{2} \mathrm{O}$, [Si IX], and $\mathrm{CO}_{2}$ lines. The solid lines indicate the Brackett line component of the best-fit spectra. 


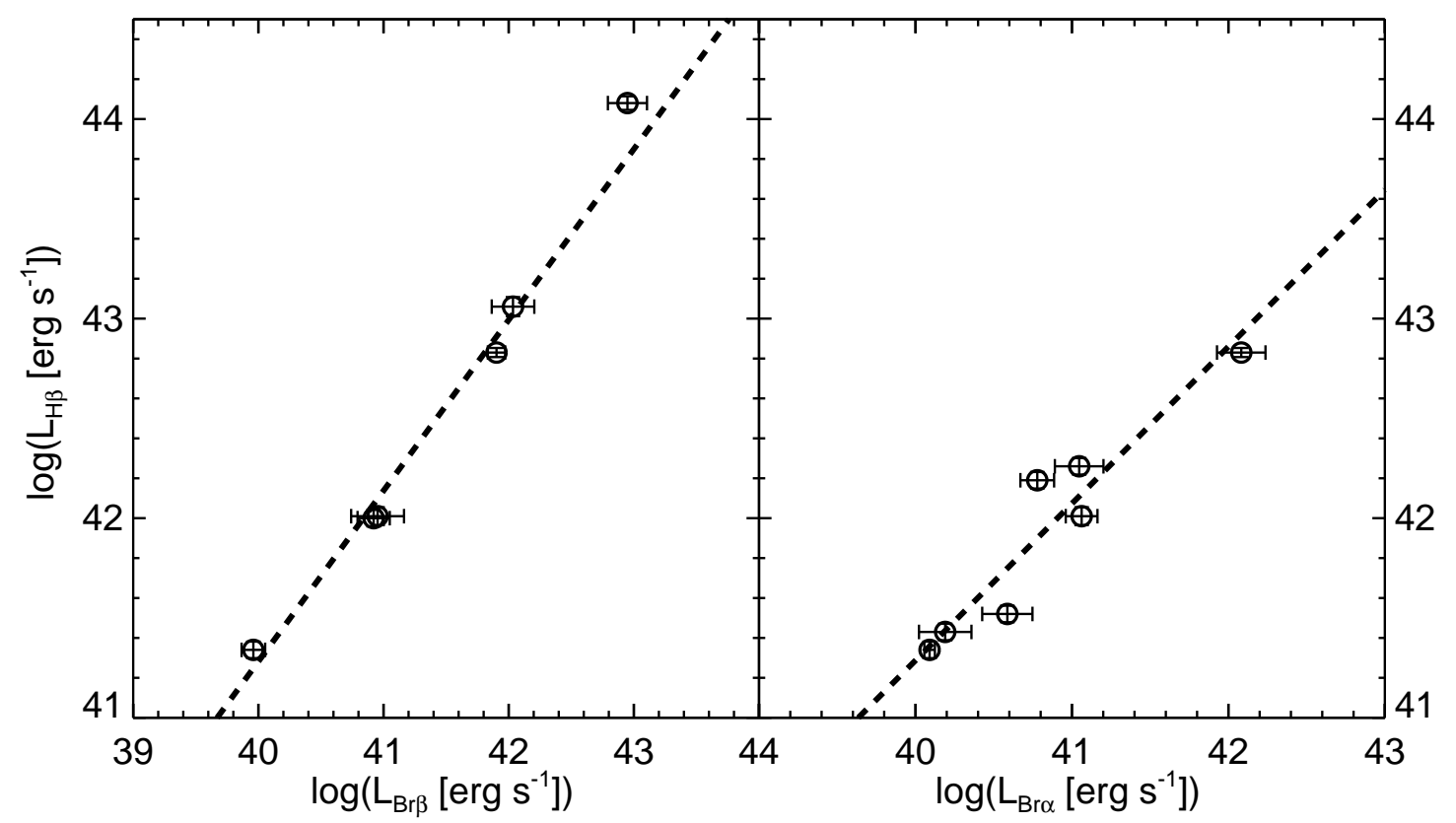

Figure 8. Comparison of the line luminosities of the Brackett lines vs. the $\mathrm{H} \beta$ line. The dotted line indicates the best-fit correlation between the luminosities of the $\mathrm{H} \beta$ and the Brackett lines.

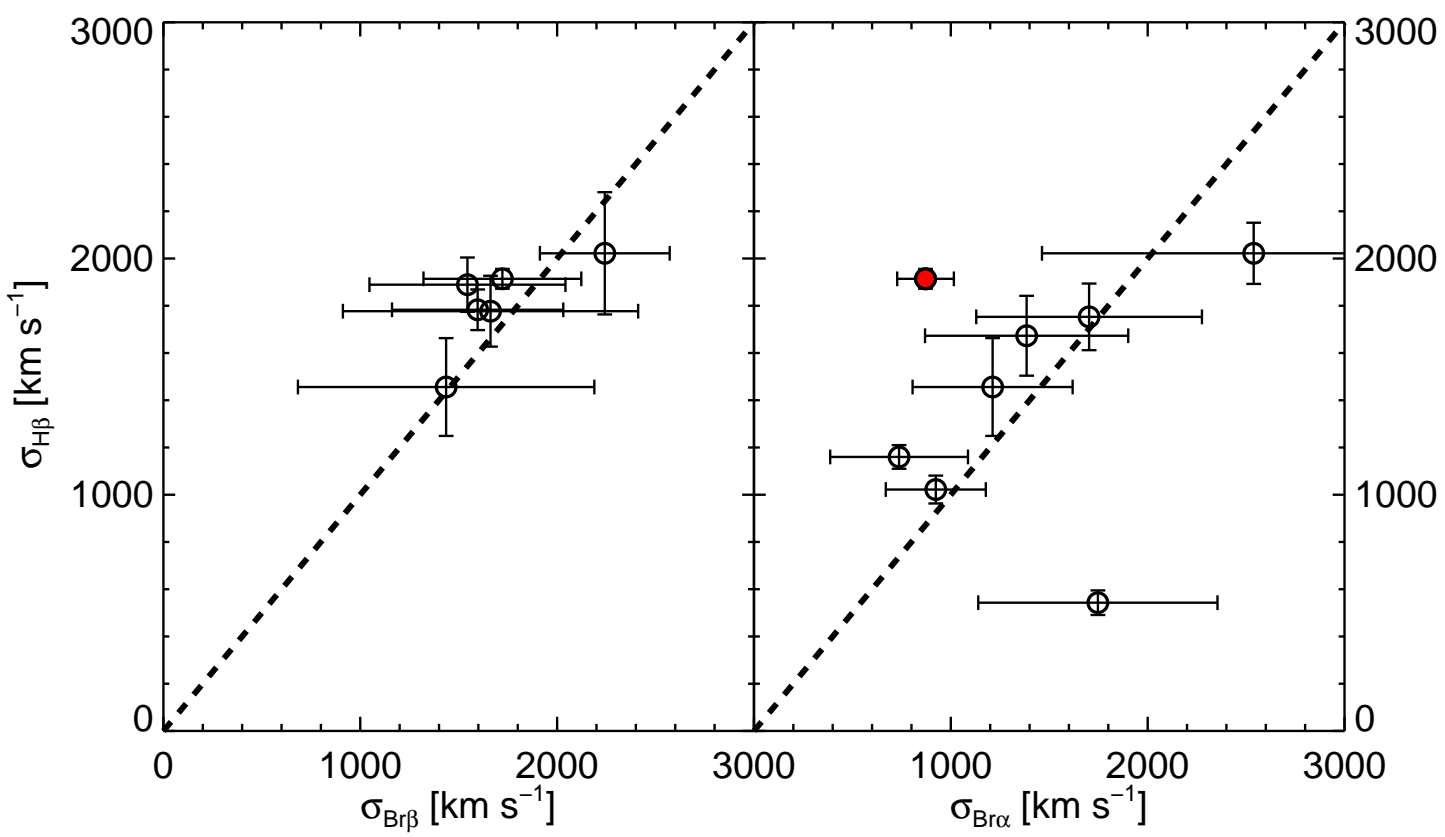

Figure 9. Comparison of the $\sigma$ widths of the Brackett lines vs. those of the $\mathrm{H} \beta$ line. The dashed line indicates the case where the $\sigma$ value of the $\mathrm{H} \beta$ and the Brackett lines are identical. The weak correlation for $\mathrm{Br} \alpha$ is likely to be due to low $\mathrm{S} / \mathrm{N}$ and the $\mathrm{CO} 2$ absorption feature right next to the Br $\alpha$ line. The $\mathrm{CO}_{2}$ absorption is the most prominently seen in the spectrum of NGC 4151, which is represented by the red filled circle. 
detected AGNs, the $3.3 \mu \mathrm{m}$ PAH emission line is detected in only two objects (NGC 4051 and NGC 7469) using the extraction width adopted in this study (Kim et al. in prep). The expected star formation contributions of the $\mathrm{Br} \alpha$ line based on the $3.3 \mu \mathrm{m}$ PAH line are $8 \%$ and $46 \%$ for NGC 4051 and NGC 7469, respectively. This assumes the above $10 \%$ flux ratio of $L_{\mathrm{Br} \alpha}$ to $L_{3.3 \mathrm{PAH}}$, but the relation contains a large scatter of a factor of two to three. Therefore, we conclude that the contribution of star formation to the Bracket emission lines is negligible (no or weak detection of PAHs) for most of the AGNs in our sample. NGC 7469 is an exceptional case with a very strong $3.3 \mu \mathrm{m}$ PAH. Even in this case, the star formation contribution is less than half for $\operatorname{Br} \alpha$, but we caution possible over-estimation of the line flux (134\%) and under-estimation of the line width $(80 \%)$ for NGC 7469.

The measured values of the $\sigma$ and luminosities of the Brackett lines and the corresponding $\mathrm{H} \beta$ properties are presented in Table 2. Note that the $\sigma$ values are corrected for the instrumental resolution by subtracting the instrumental resolution in quadrature $\left(\sigma^{2}=\sigma_{\text {obs }}^{2}-\sigma_{\text {inst }}^{2}\right)$ from the observed values. The derivation of the line luminosities assumes a standard $\Lambda$ CDM cosmology of $H_{0}=70 \mathrm{~km}$ $\mathrm{s}^{-1} \mathrm{Mpc}^{-1}, \Omega_{m}=0.3$ and $\Omega_{\Lambda}=0.7$ (e.g., Im et al. 1997)

\subsection{Correlation between Balmer and Brackett Lines}

In this section, we will investigate how the properties of the Brackett lines correlate with those of the Balmer lines. If the two lines arise from BLRs similar to each other, then we expect their line properties to correlate well. This would justify the use of the Brackett lines as an $M_{\mathrm{BH}}$ estimator (e.g., Kim et al. 2010), just like the Balmer lines that are commonly used as $M_{\mathrm{BH}}$ estimators (Greene \& Ho 2005, 2007). For this comparison, we take the average of the multi-epoch $\sigma$ values and the luminosities of the $\mathrm{H} \beta$ line from previous studies (Kaspi et al. 2000; Marziani et al. 2003; Peterson et al. 2004; Vestergaard \& Peterson 2006; Landt et al. 2008). When there is a large discrepancy in the $\sigma$ values ( $>$ $\times 1.5)$ of $\mathrm{H} \beta$ and $\mathrm{H} \alpha$, we take the average of the two. Such a case occurred for only one object (PG 1411+442). Note that we could not find the $\mathrm{H} \beta$ line luminosity data for NGC 4051, so we only adopted a $\sigma$ value from Peterson et al. (2004) for NGC 4051.

Figures 8 and 9 show the correlations between the Brackett lines and the $\mathrm{H} \beta$ line in luminosity and $\sigma$. In Figure 8 , we compare the luminosities of the Brackett lines with those of the $\mathrm{H} \beta$ line. The Pearson correlation coefficients are 0.991 and 0.947 for $\operatorname{Br} \beta$ and $\operatorname{Br} \alpha$, respectively. By performing a linear bisector fit to these points, we find that the two quantities correlate with each other as

$$
\begin{aligned}
\log \left(\frac{L_{\mathrm{H} \beta}}{10^{42} \operatorname{erg~s}^{-1}}\right) & =(0.99 \pm 0.05) \\
& +(0.85 \pm 0.05) \log \left(\frac{L_{\mathrm{Br} \beta}}{10^{42} \mathrm{erg} \mathrm{s}^{-1}}\right),
\end{aligned}
$$

and

$$
\begin{aligned}
\log \left(\frac{L_{\mathrm{H} \beta}}{10^{42} \mathrm{erg} \mathrm{s}^{-1}}\right) & =(0.86 \pm 0.11) \\
& +(0.79 \pm 0.07) \log \left(\frac{L_{\mathrm{Br} \alpha}}{10^{42} \mathrm{erg} \mathrm{s}^{-1}}\right) .
\end{aligned}
$$

In Figure 9, the dashed line indicates a perfect correlation between the $\sigma$ values of the Brackett and $\mathrm{H} \beta$ lines. The figures show a reasonable correlation between the $\operatorname{Br} \beta$ and $\mathrm{H} \beta$ line widths, but a weak correlation between the $\operatorname{Br} \alpha$ and $\mathrm{H} \beta$ line widths. The Pearson correlation coefficients are 0.735 and 0.273 for $\operatorname{Br} \beta$ and $\operatorname{Br} \alpha$, respectively. The weak correlation in $\sigma$ for $\operatorname{Br} \alpha$ is likely due to low $\mathrm{S} / \mathrm{N}$ and the difficulty in fitting some of the Br $\alpha$ lines. The zodiacal background increases rapidly at $\lambda>4 \mu \mathrm{m}$, causing the background noise to increase for $\operatorname{Br} \alpha$. Furthermore, the existence of the $\mathrm{CO}_{2}$ absorption feature right next to $\operatorname{Br} \alpha$ makes it challenging to provide a good fit. $\mathrm{CO}_{2}$ absorption is the most prominent in the spectrum of NGC 4151, which also seems to be a significant outlier in Figure 9. The exclusion of the NGC 4151 point improves the $\sigma$ correlation for $\operatorname{Br} \alpha$, with the correlation coefficient of 0.468 .

We also compared the Brackett line luminosities to the BLR radii. For the radii of BLR, we adopted values from the time lags in the variability between the $\mathrm{H} \beta$ and the $L_{5100}$ from Bentz et al. (2013). Figure 10 shows the correlation between the Brackett line luminosities and the BLR radii. The best-fit relations are

$$
\begin{aligned}
\log \left(\frac{R_{\text {BLR }}}{\text { lt - days }}\right) & =(0.82 \pm 0.08) \\
& +(0.62 \pm 0.05) \log \left(\frac{L_{\mathrm{Br} \beta}}{10^{40} \mathrm{erg} \mathrm{s}^{-1}}\right)
\end{aligned}
$$

and

$$
\begin{aligned}
\log \left(\frac{R_{\text {BLR }}}{\mathrm{lt}-\text { days }}\right) & =(0.75 \pm 0.03) \\
& +(0.66 \pm 0.06) \log \left(\frac{L_{\mathrm{Br} \alpha}}{10^{40} \mathrm{erg} \mathrm{s}^{-1}}\right)
\end{aligned}
$$

\subsection{BH Mass Estimators with Brackett Lines}

In this section, we present $M_{\mathrm{BH}}$ estimators that are based on the $\operatorname{Br} \beta$ and $\operatorname{Br} \alpha$ lines. The idea here is that the radius and the velocity of the BLR can be inferred from the luminosity and $\sigma$ of the Brackett lines, similar to the $M_{\mathrm{BH}}$ estimators based on the Balmer of the Paschen lines. Mathematically, we need to find three unknown parameters $a, b$, and $c$ in the following equation:

$$
\log (M)=a+b \log (L)+c \log (\sigma) .
$$

For simplicity, we fix the exponent of the velocity term, $c$ to 2 , as expected in the virial theorem. The exponent of the luminosity term, $b$, is expected to be 0.5 theoretically and empirically, i.e., $R_{\mathrm{BLR}} \sim L^{0.5}$ (Dibai 1977 ; Kaspi et al. 2000; Greene \& Ho 2005; Kim et al. 2010). 


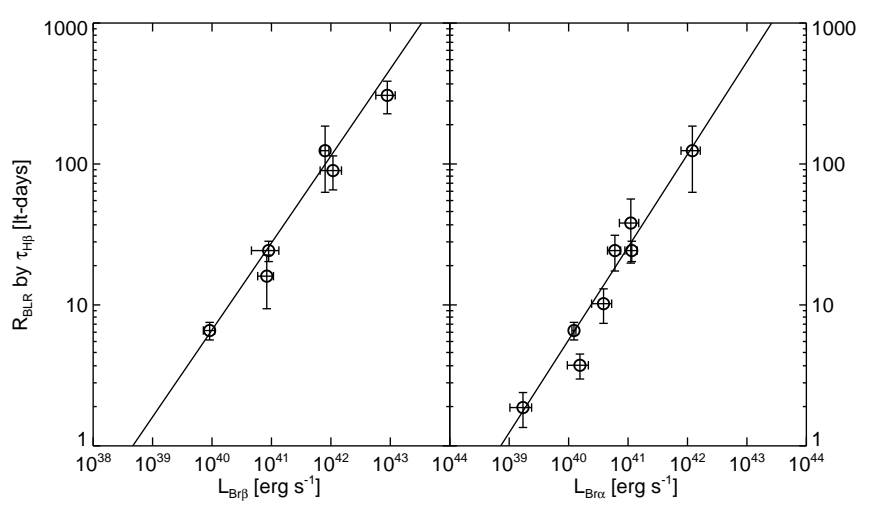

Figure 10. Comparison of the line luminosities of the Brackett lines vs. the BLR sizes. For the BLR size, we use the time lag in the variability between the $\mathrm{H} \beta$ and $L_{5100}$ from Bentz et al. (2013). The solid line indicates the best-fit correlation between the luminosities of the Brackett lines and the BLR sizes.

However, we will treat $b$ as a free parameter. In order to derive the $M_{\mathrm{BH}}$ estimators, we used the MPFITEXY routine (Williams et al. 2010) to fit a linear relationship in the logarithmic scale as in Equation (5). MPFITEXY returns the fitted parameters with the uncertainties of the parameters and an intrinsic scatter in the fitted relation. The routine has been tested against several other similar routines, and has shown to return reliable outputs (Park et al. 2012). As a result, we obtain the following relations:

$\frac{M_{\mathrm{BH}}}{M_{\odot}}=10^{6.61 \pm 0.23}\left(\frac{L_{\mathrm{Br} \beta}}{10^{40} \mathrm{erg} \mathrm{s}^{-1}}\right)^{0.68 \pm 0.13}\left(\frac{\sigma_{\mathrm{Br} \beta}}{10^{3} \mathrm{~km} \mathrm{~s}^{-1}}\right)^{2}$

and

$\frac{M_{\mathrm{BH}}}{M_{\odot}}=10^{6.68 \pm 0.20}\left(\frac{L_{\mathrm{Br} \alpha}}{10^{40} \mathrm{erg} \mathrm{s}^{-1}}\right)^{0.66 \pm 0.21}\left(\frac{\sigma_{\mathrm{Br} \alpha}}{10^{3} \mathrm{~km} \mathrm{~s}^{-1}}\right)^{2}$

In Figure 11, we plot the $M_{\mathrm{BH}}$ from the reverberation mapping method versus the $M_{\mathrm{BH}}$ from the Brackett-linebased estimators, i.e., the values from Equations (6) and (7). The rms scatters of the data points (including measurement errors) with respect to the best-fit correlations of Equations (6) and (7) are $0.229 \operatorname{dex}(\mathrm{Br} \beta)$ and 0.407 $\operatorname{dex}(\operatorname{Br} \alpha)$. The intrinsic scatter of Equation (7) is 0.284 dex, but the intrinsic scatter of Equation (6) is not determined since the fit did not require intrinsic scatter. The value of the intrinsic scatter is expected to be small $(\lesssim 0.2$ dex $)$.

Since the $\sigma$ values are rather poorly determined from the Brackett lines but we expect that they correlate closely with the $\sigma$ values of the $\mathrm{H} \beta$, we also derived the $M_{\mathrm{BH}}$ estimators using $L_{\mathrm{Br} \alpha, \beta}$ but adopting $\sigma_{\mathrm{H} \beta}$ as a proxy for $\sigma_{\mathrm{Br} \alpha, \beta}$. The $\sigma_{\mathrm{H} \beta}$ values are taken from Peterson et al. (2004). Since dust extinction in a host galaxy can reduce its line luminosity significantly but not as much for the line width, this is a plausible path to estimate $M_{\mathrm{BH}}$ of red AGNs whose $\sigma_{\mathrm{H} \beta}$ can be determined to a good accuracy, but its $\mathrm{H} \beta$ luminosity is quite uncertain due to an unknown amount of dust extinction. In this process, we also fixed the exponent of the velocity term, $c$, to 2 . The resultant $M_{\mathrm{BH}}$ estimators using
$L_{\mathrm{Br} \alpha, \beta}$ and $\sigma_{\mathrm{H} \beta}$ are given below and also in Figure 12 as

$\frac{M_{\mathrm{BH}}}{M_{\odot}}=10^{6.44 \pm 0.17}\left(\frac{L_{\mathrm{Br} \beta}}{10^{40} \mathrm{erg} \mathrm{s}^{-1}}\right)^{0.72 \pm 0.10}\left(\frac{\sigma_{\mathrm{H} \beta}}{10^{3} \mathrm{~km} \mathrm{~s}^{-1}}\right)^{2}$

and

$\frac{M_{\mathrm{BH}}}{M_{\odot}}=10^{6.76 \pm 0.17}\left(\frac{L_{\mathrm{Br} \alpha}}{10^{40} \mathrm{erg} \mathrm{s}^{-1}}\right)^{0.48 \pm 0.17}\left(\frac{\sigma_{\mathrm{H} \beta}}{10^{3} \mathrm{~km} \mathrm{~s}^{-1}}\right)^{2}$.

The raw rms scatters (including measurement errors) with respect to a perfect correlation between the reverberation-mapping-based $M_{\mathrm{BH}}$ and $M_{\mathrm{BH}}$ for Equations (8) and (9) are $0.203 \operatorname{dex}(\operatorname{Br} \beta)$ and $0.364 \operatorname{dex}$ $(\operatorname{Br} \alpha)$, while the intrinsic scatters are $0.152 \operatorname{dex}(\operatorname{Br} \beta)$ and $0.310 \operatorname{dex}(\operatorname{Br} \alpha)$. However, the intrinsic scatters are not better than the values for the purely Brackett-based $M_{\mathrm{BH}}$ estimators in Equations (6) and (7).

Since the $\sigma$ values of Brackett lines, especially for $\operatorname{Br} \alpha$, show a weak correlation, we test whether or not the $\sigma$ values of Brackett lines are adding useful information to the $M_{\mathrm{BH}}$ estimators. For this test, we derive $M_{\mathrm{BH}}$ estimators using only Brackett line luminosities:

$$
\frac{M_{\mathrm{BH}}}{M_{\odot}}=10^{6.92 \pm 0.26}\left(\frac{L_{\mathrm{Br} \beta}}{10^{40} \mathrm{erg} \mathrm{s}^{-1}}\right)^{0.73 \pm 0.15}
$$

and

$$
\frac{M_{\mathrm{BH}}}{M_{\odot}}=10^{6.79 \pm 0.15}\left(\frac{L_{\mathrm{Br} \alpha}}{10^{40} \mathrm{erg} \mathrm{s}^{-1}}\right)^{0.76 \pm 0.14} .
$$

Figure 13 shows the $M_{\mathrm{BH}}$ values from the reverberation mapping method versus the $M_{\mathrm{BH}}$ values from the $M_{\mathrm{BH}}$ estimators using only $L_{\mathrm{Br} \beta}$ or $L_{\mathrm{Br} \alpha}$, i.e., the values from Equations (10) and (11). We find that the intrinsic 2 scatters in these relations are $0.314 \operatorname{dex}(\operatorname{Br} \beta)$, and 0.251 - $\operatorname{dex}(\operatorname{Br} \alpha)$. Therefore, for $\operatorname{Br} \beta$, the intrinsic scatter in the $M_{\mathrm{BH}}$ estimators becomes smaller as we move from the luminosity-only estimator to the full $\operatorname{Br} \beta$ luminosity $+\sigma$ estimators, lending support to the usefulness of Equation (6). On other hand, the same cannot be said for the $\operatorname{Br} \alpha$ based estimators. This suggests that $\operatorname{Br} \alpha \sigma$ measurement is more challenging with the current data, and the evaluation of the $\operatorname{Br} \alpha$-based $M_{\mathrm{BH}}$ estimators is needed with data sets with better sensitivity. Considering that the Eddington ratio of AGNs used for Equations (10) and (11) are between 0.015 and 0.225, Equations (10) and (11) could be useful when (i) the Brackett line luminosities are available but not for reliable $\sigma$ values and (ii) the Eddington ratio of the sample is within a range of 0.015 to 0.225 .

\subsection{Line Luminosity Ratios}

We investigate the physical conditions of BLR by comparing the observed line luminosity ratios of the Balmer through Brackett lines to the line luminosity ratios computed from the CLOUDY code (version 10.00; Ferland et al. 1998). The line luminosity ratios are computed by varying three physical parameters - the shape of the ionizing continuum $(\alpha=-1.0,-1.5)$, the ionization parameter $\left(U=10^{-5.5}-10^{0.5}\right)$, and the hydrogen density $\left(n=10^{9}-10^{13} \mathrm{~cm}^{-3}\right)$. The hydrogen number 


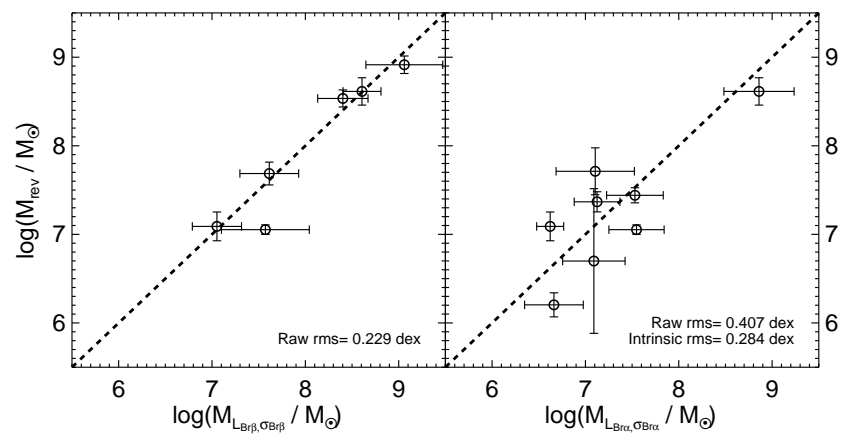

Figure 11. Comparison of the Brackett-line-based $M_{\mathrm{BH}}$ vs. $M_{\mathrm{BH}}$ derived from the reverberation mapping method. The Brackett-linebased $M_{\mathrm{BH}}$ is derived by using the $L$ and $\sigma$ of the Brackett lines as Equations (6) and (7). The dashed line indicates a line where $M_{\mathrm{BH}}$ from Brackett lines and the reverberation mapping methods are identical. In each panel, we indicate the intrinsic and the raw (no correction of the measurement errors) scatters of the points with respect to the dashed line.

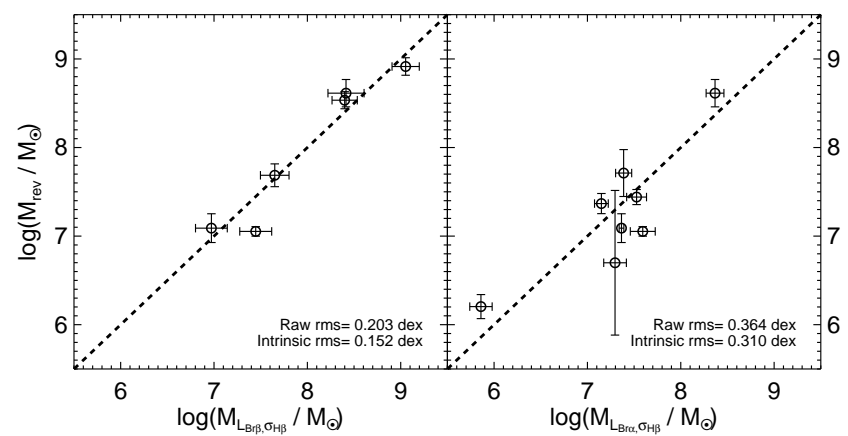

Figure 12. Comparison of $M_{\mathrm{BH}}$ from Equations (8) and (9) vs. $M_{\mathrm{BH}}$ derived from the reverberation mapping method. The Equations (8) and (9) are established by using the $L_{\mathrm{Br} \alpha, \beta}$ but adopting $\sigma_{\mathrm{H} \beta}$. The meaning of the line is identical to Figure 11.

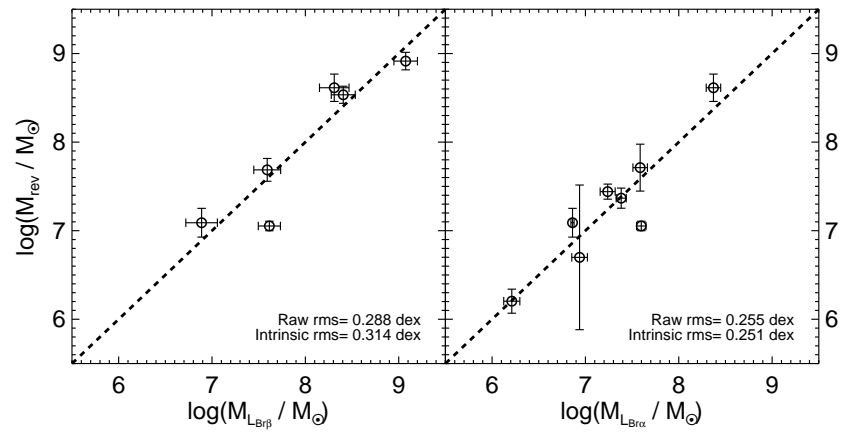

Figure 13. Comparison of $M_{\mathrm{BH}}$ from Equations (10) and (11) vs. $M_{\mathrm{BH}}$ derived from the reverberation mapping method. The Equations (10) and (11) are established by using only $L_{\mathrm{Br} \alpha, \beta}$. The meanings of the line is identical to Figure 11.

densities are commonly taken as $n \simeq 10^{9}-10^{10} \mathrm{~cm}^{-3}$ for type-1 AGNs (Davidson \& Netzer 1979; Rees et al. 1989; Ferland \& Persson 1989), but there are studies suggesting a high hydrogen number density $\left(n>10^{12} \mathrm{~cm}^{-3}\right)$ with a low ionization parameter of $U=10^{-5}$ (e.g., Ruff et al. 2012) for BLR.

Figure 14 shows the observed line luminosity ratios (with respect to $\mathrm{H} \beta$ ) as a function of wavelength for seven AGNs, for which we have at least one Brackett to Balmer line ratio and an additional line ratio of either Paschen or Brackett lines. The line luminosities are corrected for galactic extinction with the York Extinction Solver (McCall 2004), adopting $E(B-V)$ values from (Schlafly \& Finkbeiner 2011) and $R_{V}=3.07$. No attempt is made to correct for internal dust ex- tinction. Note that the Paschen line ratios are taken from Kim et al. (2010). The mean line ratios of the seven AGNs are $L_{\mathrm{Br} \alpha} / L_{\mathrm{Br} \beta}=1.37 \pm 0.31, L_{\mathrm{Br} \beta} / L_{\mathrm{H} \beta}=$ $0.073 \pm 0.010$, and $L_{\mathrm{Br} \alpha} / L_{\mathrm{H} \beta}=0.081 \pm 0.014$.

In order to find the most likely physical condition for each object, we searched for a parameter set that minimizes $\chi^{2}$, which is a function of line ratios such as $L_{\mathrm{P} \beta} / L_{\mathrm{H} \beta}, L_{\mathrm{P} \alpha} / L_{\mathrm{H} \beta}, L_{\mathrm{Br} \beta} / L_{\mathrm{H} \beta}$, and $L_{\mathrm{Br} \alpha} / L_{\mathrm{H} \beta}$ following the approach by Ruff et al. (2012):

$$
\chi^{2}=\sum_{i=1}^{N} \frac{\left(R_{\text {observed }, i}-R_{\text {model }, i}\right)^{2}}{\sigma_{i}^{2}},
$$

where $N$ is the number of line luminosity ratios, and two types of $R_{i}$ are the line luminosity ratios from either 

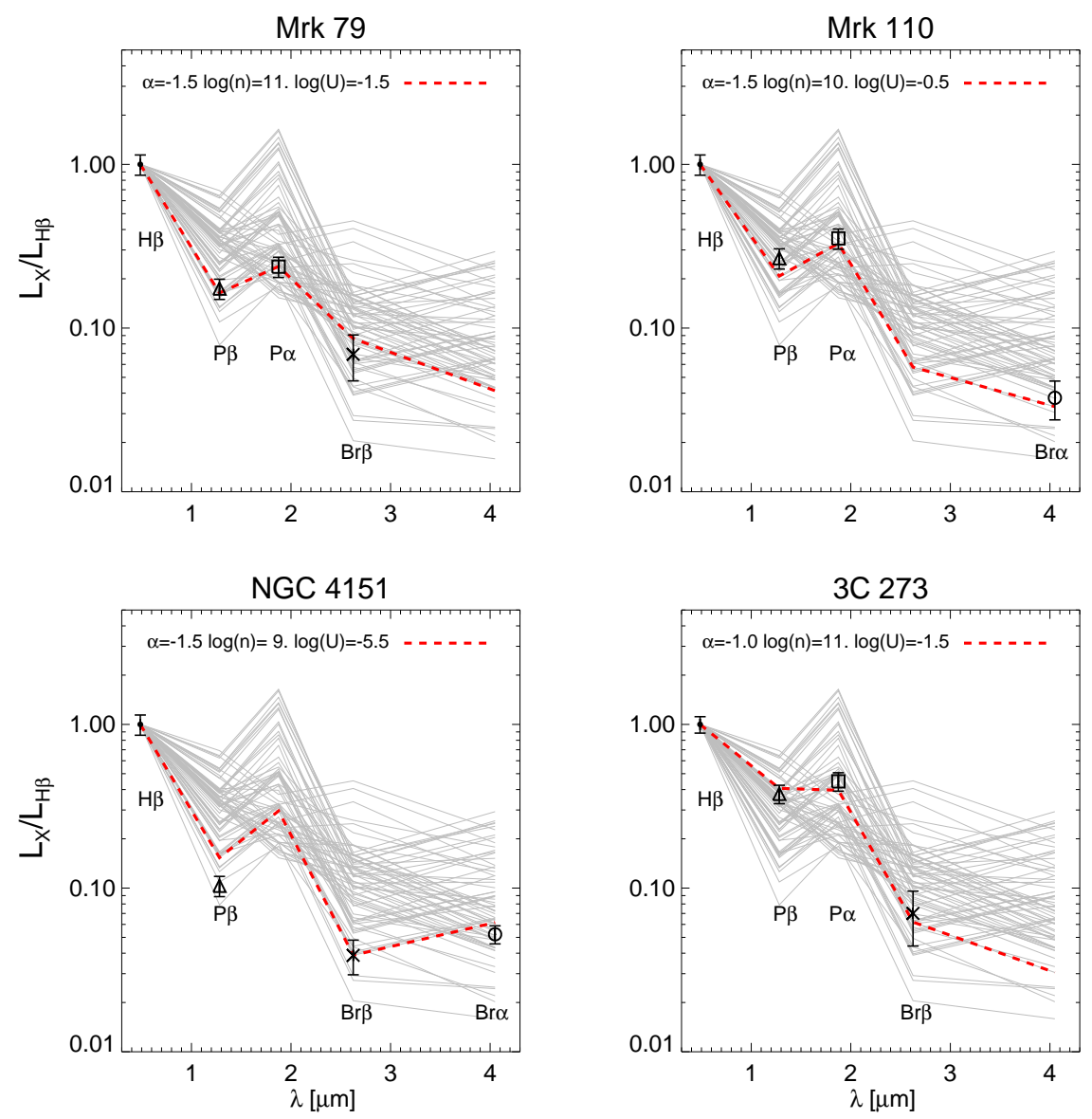

Figure 14. Line luminosity ratios of Paschen and Brackett with respect to $H \beta$. The crosses and the circles represent AGNs in the reverberation mapped sample with the Brackett line luminosity measurements. When available, we also plot the line luminosity ratios for $\mathrm{P} \alpha$ (squares) and $\mathrm{P} \beta$ (triangles) with respect to $\mathrm{H} \beta$ from Kim et al. (2010). The gray lines represent the expected line ratios from the CLOUDY code, assuming various parameters with different combinations of $\alpha=-1.0 \sim-1.5, n=10^{9} \sim 13 \mathrm{~cm}^{-3}$, and $U=10^{-5.5 \sim 0.5}$. In each panel, we indicate the most likely set of the parameters, with the CLOUDY model predictions for such a parameter set in the red dashed line.

Table 3

BLR Conditions

\begin{tabular}{cccc}
\hline \hline Object Name & $\alpha$ & $\begin{array}{c}\mathrm{n} \\
{\left[\mathrm{cm}^{-3}\right]}\end{array}$ & $\mathrm{U}$ \\
& & & \\
\hline Mrk 79 & -1.5 & $10^{11}$ & $10^{-1.5}$ \\
Mrk 110 & -1.5 & $10^{10}$ & $10^{-0.5}$ \\
NGC 4151 & -1.5 & $10^{9}$ & $10^{-5.5}$ \\
3C 273 & -1.0 & $10^{11}$ & $10^{-1.5}$ \\
NGC 4593 & -1.0 & $10^{11}$ & $10^{-2.5}$ \\
PG 1411+442 & -1.5 & $10^{9}$ & $10^{-1.5}$ \\
NGC 7469 & -1.5 & $10^{11}$ & $10^{-5.5}$ \\
& & & \\
\hline
\end{tabular}

observation (when with a subscript, "observed") or the CLOUDY model (with a subscript, "model"), and $\sigma_{i}$ is the error in the measured line ratio. The best-fit parameter sets are indicated for each object in Figure 14, and are summarized in Table 3 . The best-fit line luminosity ratios are also plotted as a red dashed line in Figure 14.

The CLOUDY models can reproduce the observed line luminosity ratios, under commonly cited BLR physical conditions of $n=10^{9}-10^{11} \mathrm{~cm}^{-3}$ and $U=10^{-2.5}$ $10^{0.5}$, suggesting that most of the objects with Brackett line detections do not require a more exotic condition $\left(n>10^{12} \mathrm{~cm}^{-3}\right.$ and $\left.U<10^{-5}\right)$, such as that found in Ruff et al. (2012). One exception is NGC 7469, for which we find a best-fit parameter set of $n=10^{11} \mathrm{~cm}^{-3}$ and $U=10^{-5.5}$. This is the case where the Brackett line luminosity may be affected by the star formation component (possibly by as much as 50\%), and thus the interpretation of the line luminosity ratio result cannot be taken too seriously for this object.

\section{DUST COMPONENT}

In this section, we present hot ( $>1000 \mathrm{~K}$; e.g., Barvainis 1987; Glikman et al. 2006) and warm ( $200 \mathrm{~K}$; e.g., Netzer et al. 2007; Deo et al. 2009) dust temperatures and luminosities of the PG QSOs and the reverberation-mapped AGNs, and we investigate the correlation between hot dust luminosity $\left(L_{\mathrm{HD}}\right)$ and $L_{\mathrm{bol}}$. For this purpose, we restrict our analysis to luminous AGNs with $L_{\text {bol }}>10^{45.7} \mathrm{erg} \mathrm{s}^{-1}$ for which the host galaxy light contribution is less than $10 \%$ at $0.51 \mu \mathrm{m}$ (Shen et al. 2011), and even smaller at NIR.

To study the AGN dust components, we assembled a multi-wavelength data set from the optical to MIR by obtaining photometry of the objects from SDSS, 2MASS, $W I S E$ (W3 and $W 4)$, and ISO $(7.3 \mu \mathrm{m}$ : Haas et al. 

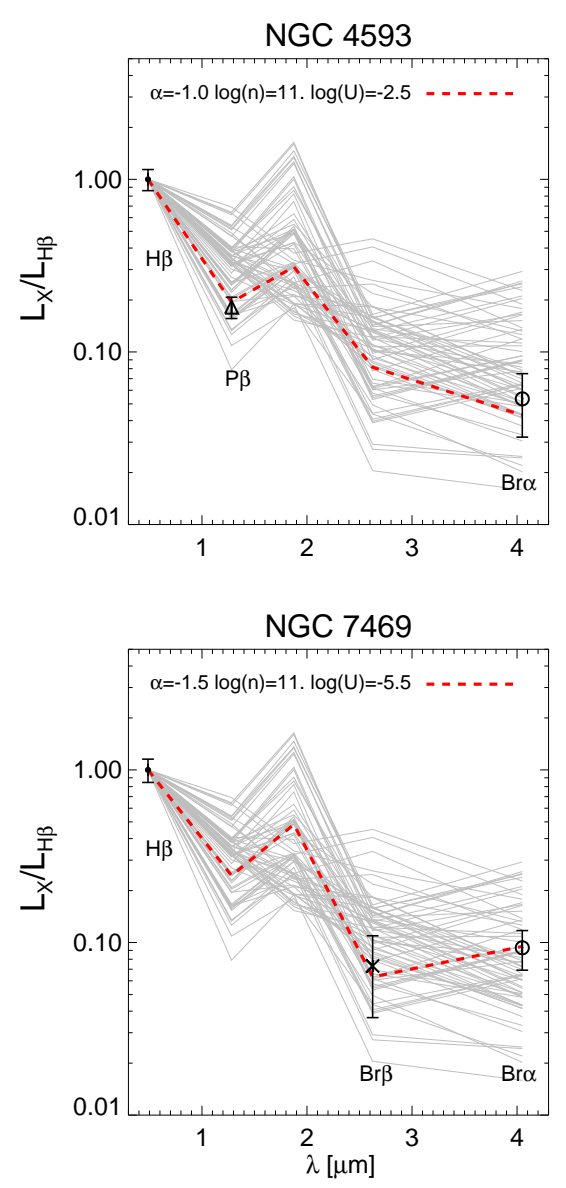

Figure 14. Continued

2003). These multi-wavelength photometric data are combined with the $A K A R I$ spectra, and we fit them with a SED model that is composed of a single power law and a double black body radiation component as

$$
F_{\nu}=C_{0} \nu^{\alpha}+C_{1} B_{\nu}\left(T_{\mathrm{HD}}\right)+C_{2} B_{\nu}\left(T_{\mathrm{WD}}\right),
$$

where $\alpha$ is the slope of the underlying power-law continuum which dominates the optical light, $B_{\nu}$ is the Planck function as a function of $\nu, T_{\mathrm{HD}}$ and $T_{\mathrm{WD}}$ are the hot and warm dust temperatures, and $C_{0}, C_{1}$, and $C_{2}$ are the normalization constants of each component. The fitting result gives us the luminosities and the temperatures of the hot/warm dust components.

We note three technical points that we took into account during the fit. First, we excluded SDSS photometry between 0.6 and $0.7 \mu \mathrm{m}$ in the rest frame to avoid the inclusion of the $\mathrm{H} \alpha$ line which generally has a large equivalent width and produces a bump in the SED that deviates from a power law which is assumed as the continuum shape in the optical. Second, we increased the errors of the SDSS data points to represent the deviation of the optical continuum shape from a power law. Here, the deviation is caused by the intrinsic fluctuations in the continuum spectra due to Fe emission complex, emission lines other than $\mathrm{H} \alpha$, and the host galaxy light. Without increasing the error, a fit to the SDSS photometry points with a simple power-law function ends up with a rather large reduced $\chi^{2}$ values due to very small original SDSS photometry errors. From a composite spectrum of
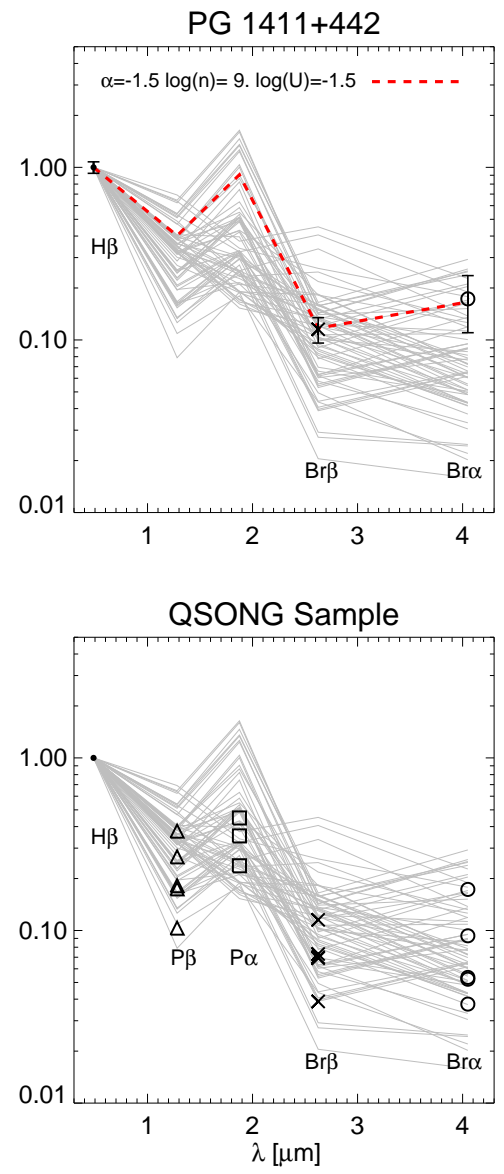

a quasar (Glikman et al. 2006) placed at $0 \leq z \leq 0.4$, we find the intrinsic fluctuation of the optical spectrum with respect to a simple featureless power law to be $\sigma_{m}=0.035 \mathrm{mag}$, and this value is added in quadrature to the original SDSS photometry error in each band. Third, we calculate the reduced chi-square, $\chi_{\nu}^{2}$ of the fit, and the hot and warm dust properties are taken seriously only if $\chi_{\nu}^{2}<5$. One object (PG 1322+659) is excluded by the $\chi_{\nu}^{2}$ limit for which we had difficulty fitting the shape of the AKARI spectrum (the last object in Figure 15).

Through the procedure above, we obtain the temperatures and luminosities of the hot and warm dust components of 19 AGNs (2 reverberation mapped AGNs and 17 PG QSOs). The fitting results are presented in Figure 15, showing that both the hot and warm dust components exist and contribute to the NIR and MIR spectra of AGNs. However, four objects (PG 1114+445, PG 1116+215, PG 1543+489, and PG 1545+210) show an excess of $7.3 \mu \mathrm{m}$ ISO photometry. This excess can hint at the existence of an intermediate-temperature dust component or a continuous dust temperature.

The SED fit provides formal fitting errors of $0.8 \%$ in $T_{\mathrm{HD}}$ and $3.1 \%$ in $L_{\mathrm{HD}}$, where we obtain a small error in $T_{\mathrm{HD}}$ due to the strong constraints provided by the AKARI spectra. The errors in Table 4 indicate the formal fitting errors. Since the changes in the assumptions that went into the fitting model could influence the result more than the formal fitting errors, we ex- 

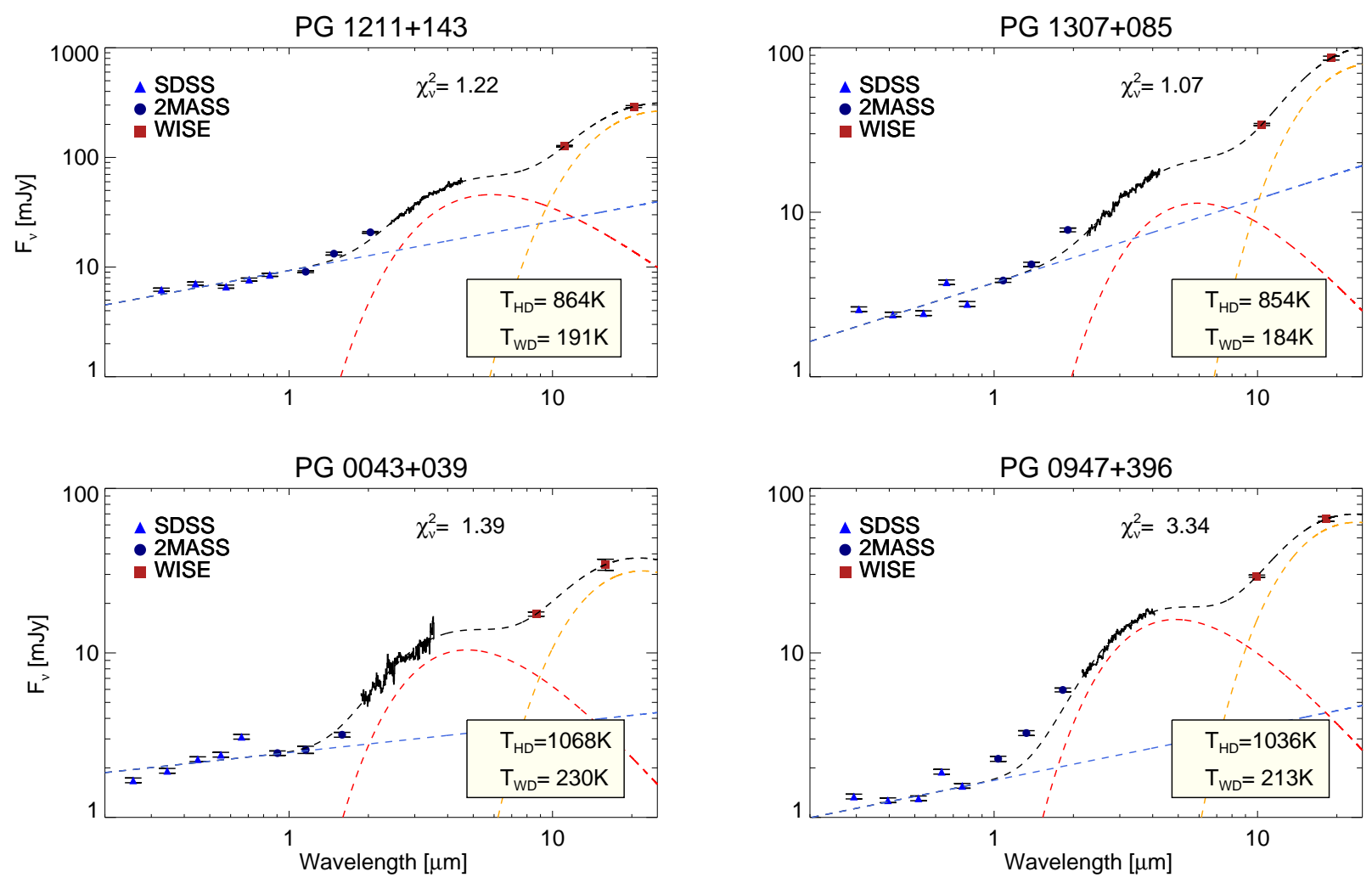

Figure 15. Photometric and spectroscopic SED. The SEDs are shown in rest-frame. Triangle, circle, square, and star indicate photometric data point from SDSS, 2MASS, WISE $(W 3$ and $W 4)$, and $I S O(7.3 \mu \mathrm{m})$, respectively. The black solid line denotes $A K A R I$ spectrum. The dashed lines are fitted results. The blue, red, and yellow dashed lines mean power-law, hot, and warm dust components, respectively, and the black dashed line is the sum of all components. The measured hot and warm dust temperatures are indicated in the lower right-hand corner. The dust properties are taken only if $\chi_{\nu}^{2}<5$, and the $\chi_{\nu}^{2}$ of each sample is indicated at top of each figure. The PG $1322+659$ is excluded by the $\chi_{\nu}^{2}$ limit, and the SED of the PG $1322+659$ is indicated in last panel of this figure.

Table 4

Dust Components Fitting Results

\begin{tabular}{ccccccc}
\hline \hline Objects & $\begin{array}{c}T_{\mathrm{HD}} \\
{[\mathrm{K}]}\end{array}$ & $\begin{array}{c}T_{\mathrm{WD}} \\
{[\mathrm{K}]}\end{array}$ & $\begin{array}{c}\log \left(L_{\mathrm{HD}}\right) \\
{\left[\mathrm{erg} \mathrm{s}^{-1}\right]}\end{array}$ & $\begin{array}{c}\log \left(L_{\mathrm{WD}}\right) \\
{\left[\mathrm{erg} \mathrm{s}^{-1}\right]}\end{array}$ & $\mathrm{CF}_{\mathrm{HD}}$ & $\alpha$ \\
\hline PG 1211+143 & $864 \pm 5$ & $191 \pm 2$ & $45.27 \pm 0.01$ & $45.38 \pm 0.02$ & 0.28 & $-1.55 \pm 0.02$ \\
PG 1307+085 & $854 \pm 13$ & $184 \pm 3$ & $45.27 \pm 0.03$ & $45.45 \pm 0.03$ & 0.18 & $-1.49 \pm 0.02$ \\
PG 0043+039 & $1068 \pm 14$ & $230 \pm 9$ & $46.22 \pm 0.02$ & $46.04 \pm 0.07$ & 0.23 & $-1.83 \pm 0.03$ \\
PG 0947+396 & $1036 \pm 2$ & $213 \pm 3$ & $45.78 \pm 0.00$ & $45.68 \pm 0.03$ & 0.60 & $-1.68 \pm 0.01$ \\
PG 1048+342 & $1032 \pm 20$ & $213 \pm 5$ & $45.06 \pm 0.03$ & $45.12 \pm 0.04$ & 0.25 & $-1.55 \pm 0.04$ \\
PG 1049-005 & $1068 \pm 6$ & $222 \pm 2$ & $46.48 \pm 0.01$ & $46.65 \pm 0.02$ & 0.43 & $-1.68 \pm 0.03$ \\
PG 1100+772 & $1062 \pm 9$ & $215 \pm 3$ & $46.20 \pm 0.02$ & $46.14 \pm 0.03$ & 0.29 & $-1.89 \pm 0.04$ \\
PG 1103-006 & $1144 \pm 17$ & $250 \pm 5$ & $46.33 \pm 0.03$ & $46.37 \pm 0.03$ & 0.35 & $-1.95 \pm 0.05$ \\
PG 1114+445 & $917 \pm 2$ & $195 \pm 2$ & $45.56 \pm 0.00$ & $45.73 \pm 0.02$ & 0.51 & $-1.50 \pm 0.01$ \\
PG 1116+215 & $1078 \pm 1$ & $216 \pm 3$ & $46.17 \pm 0.00$ & $45.84 \pm 0.03$ & 0.37 & $-2.00 \pm 0.02$ \\
PG 1121+422 & $1078 \pm 15$ & $236 \pm 9$ & $45.55 \pm 0.02$ & $45.11 \pm 0.06$ & 0.21 & $-1.95 \pm 0.04$ \\
PG 1259+593 & $1326 \pm 1$ & $315 \pm 8$ & $46.81 \pm 0.00$ & $46.32 \pm 0.04$ & 0.41 & $-2.15 \pm 0.01$ \\
PG 1402+261 & $1106 \pm 3$ & $219 \pm 2$ & $45.95 \pm 0.00$ & $45.79 \pm 0.02$ & 0.64 & $-2.23 \pm 0.01$ \\
PG 1543+489 & $1231 \pm 2$ & $239 \pm 2$ & $46.58 \pm 0.00$ & $46.81 \pm 0.02$ & 0.83 & $-1.98 \pm 0.01$ \\
PG 1545+210 & $1151 \pm 8$ & $209 \pm 3$ & $46.03 \pm 0.00$ & $45.82 \pm 0.03$ & 0.29 & $-2.17 \pm 0.04$ \\
PG 1704+608 & $1145 \pm 3$ & $191 \pm 2$ & $46.63 \pm 0.01$ & $46.67 \pm 0.03$ & 0.36 & $-1.55 \pm 0.01$ \\
PG 2112+059 & $1172 \pm 7$ & $263 \pm 4$ & $46.91 \pm 0.00$ & $46.82 \pm 0.03$ & 0.52 & $-1.75 \pm 0.03$ \\
PG 2233+134 & $1111 \pm 7$ & $224 \pm 3$ & $46.08 \pm 0.01$ & $46.26 \pm 0.02$ & 0.40 & $-2.25 \pm 0.05$ \\
PG 2308+098 & $1142 \pm 10$ & $177 \pm 17$ & $46.29 \pm 0.02$ & $46.08 \pm 0.15$ & 0.19 & $-1.50 \pm 0.03$ \\
& & & & & & \\
\hline
\end{tabular}



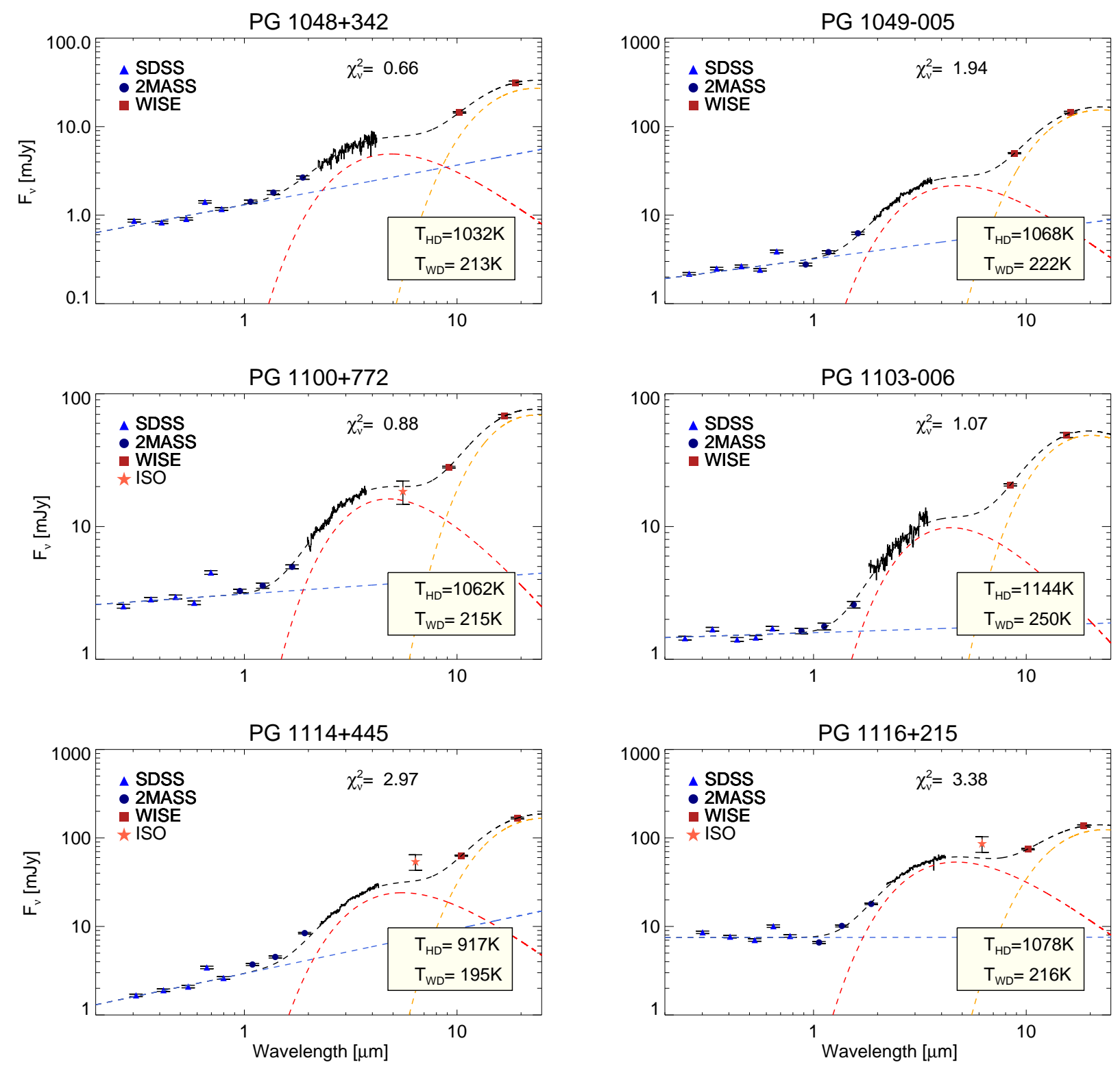

Figure 15. Continued 

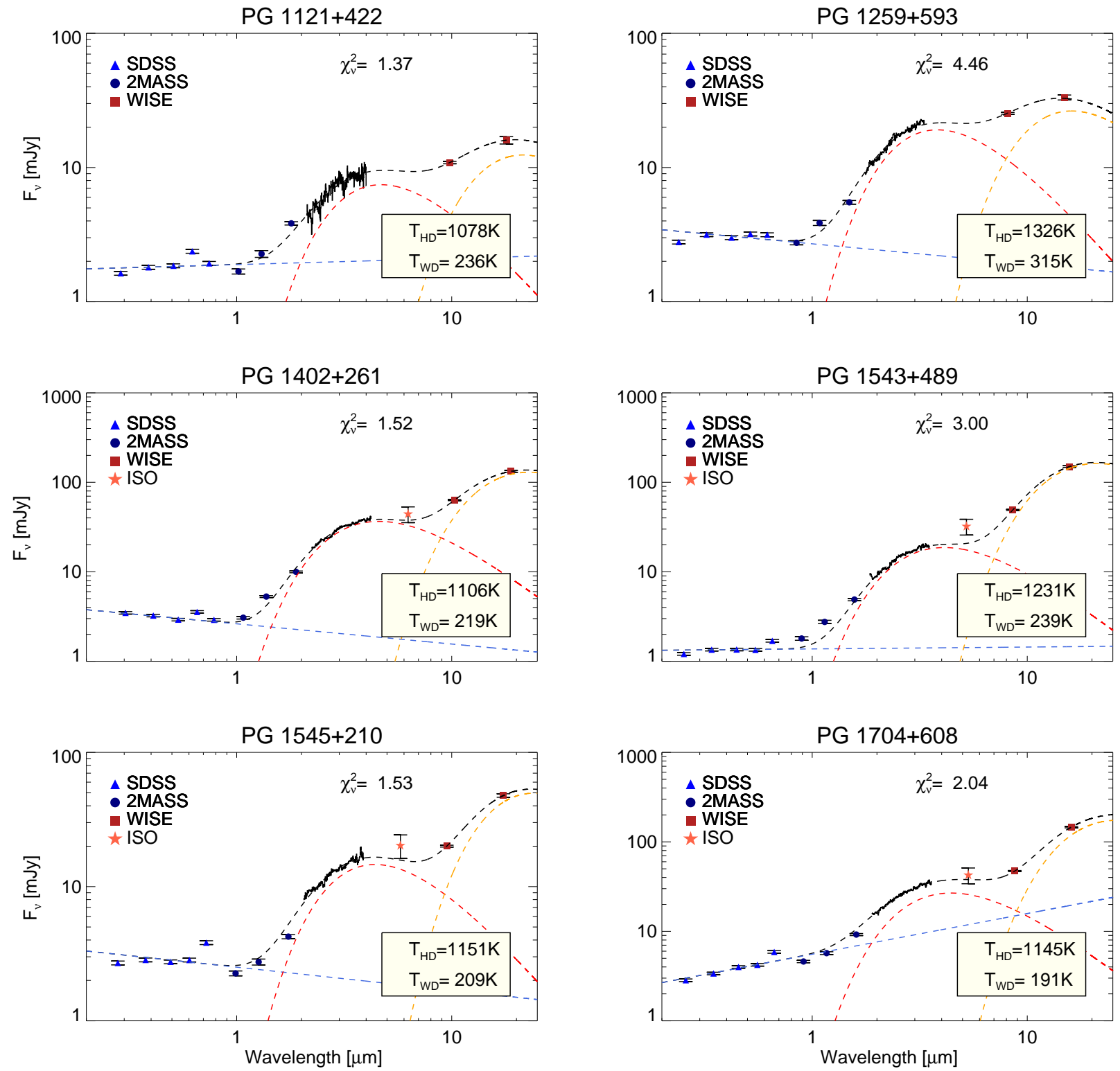

Figure 15. Continued

plored how the derived quantities could be affected by changing the following conditions in the fit as an attempt to estimate more realistic errors: (1) fixing the powerlaw slope of the continuum, $\alpha$, in Equation (13) to a value that deviates by five times the formal error from the fit; (2) the reduction of the fluxes of the $A K A R I$ data by $10 \%$, since $A K A R I$ fluxes could be overestimated by that amount (Figure 3 ), and (3) the subtraction of the host galaxy contribution. The mean absolute differences in the fitted quantities from the change in the power law slope are $3.3 \%\left(T_{\mathrm{HD}}\right.$; between $0.6 \%$ and $8.7 \%$ ) and $11 \%\left(L_{\mathrm{HD}}\right.$; between $2.6 \%$ and $36 \%$ ), and from the flux of $A K A R I$ data are $2.7 \%$ (between $<1 \%$ and $6.5 \%$ ) in $T_{\mathrm{HD}}$ and $15 \%$ (between $11 \%$ and $22 \%$ ) in $L_{\mathrm{HD}}$. The reduction in the $A K A R I$ flux leads to the reduction in $T_{\mathrm{HD}}$ and $L_{\mathrm{HD}}$. We note that the change of the power-law slope and the $A K A R I$ flux value keeps the $\chi_{\nu}^{2}$ value to less than five, except for one object. We esti- mated the contribution of the host galaxy light in $L_{5100}$ (Peterson et al. 2004; Vestergaard \& Peterson 2006) using Equation (1) of Shen et al. 2011, and subtracted the host galaxy light assuming an elliptical galaxy SED shape (Bruzual \& Charlot 2003) with the conditions of solar metallicity, $\tau=0.1$ Gyr, $\mathrm{t}=10 \mathrm{Gyr}$, and a Chabrier IMF. We examined how the fitting result changes with the subtraction of the host galaxy component, and find mean differences of $1.5 \%$ (between $<1 \%$ and $7.5 \%$ ) in $T_{\mathrm{HD}}$ and $7.5 \%$ (between $<1 \%$ and $22 \%$ ) in $L_{\mathrm{HD}}$, where $T_{\mathrm{HD}}$ decreases and $L_{\mathrm{HD}}$ increases in most cases. With these considerations, the error of $T_{\mathrm{HD}}$ is realistically $\sim 4 \%$, while the error in $L_{\mathrm{HD}}$ could be as much as $\sim 14 \%$. For the warm dust component, we have only two photometric data points for most AGNs and a single point in some AGNs; we caution that the fitted result have larger errors and can be affected more easily by the fitting method than the hot dust component. 

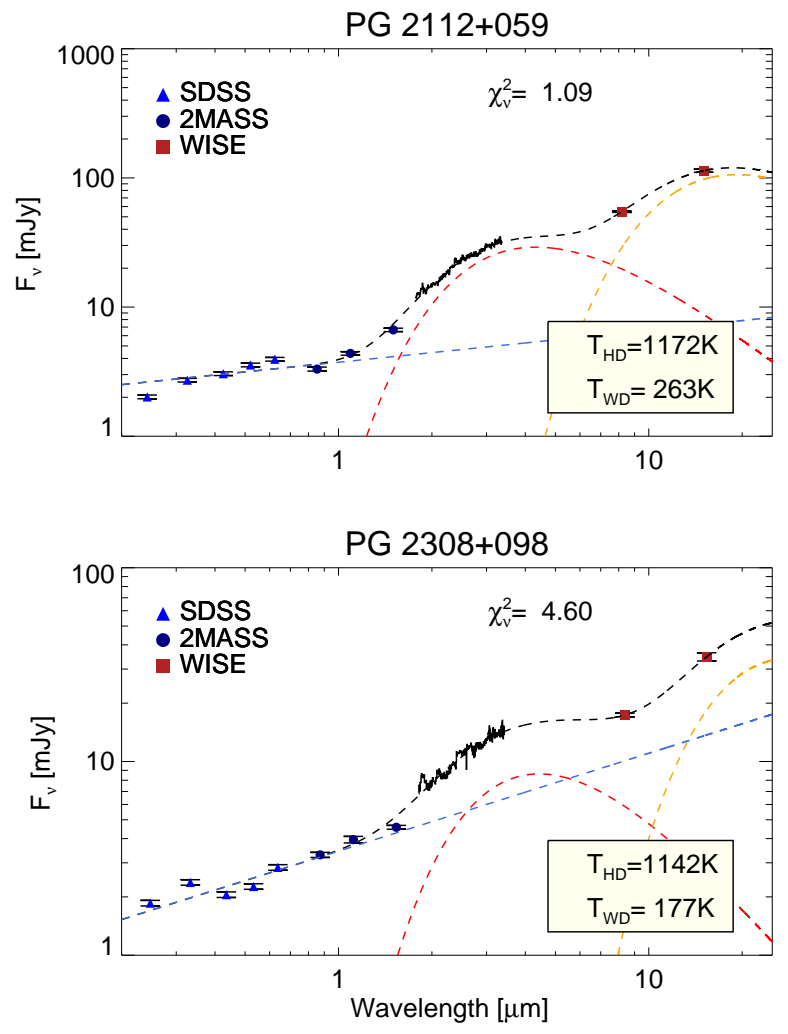

Figure 15. Continued

The left panel of Figure 16 shows $L_{\mathrm{bol}}$ versus $T_{\mathrm{HD}}$. The $L_{\mathrm{bol}}$ values are estimated from $L_{5100}$ using a bolometric correction factor of 10.3 (Richards et al. 2006), and the $L_{5100}$ values are taken from Bentz et al. (2009) and Vestergaard \& Peterson (2006). The $L_{\mathrm{bol}}$ and the $T_{\mathrm{HD}}$ values have a positive correlation as

$$
\frac{T_{\mathrm{HD}}}{1800 \mathrm{~K}}=10^{-0.231 \pm 0.003}\left(\frac{L_{\mathrm{bol}}}{10^{46} \mathrm{erg} \mathrm{s}^{-1}}\right)^{0.061 \pm 0.005}
$$

and the Pearson correlation coefficient is 0.690 . We find that the mean $T_{\mathrm{HD}}$ and $T_{\mathrm{WD}}$ are $1083 \mathrm{~K}$ and $221 \mathrm{~K}$, respectively, which is lower than the commonly cited $T_{\mathrm{HD}}$ of $1500 \mathrm{~K}$ (e.g., Barvainis 1987; Elvis et al. 1994), and somewhat lower than the value quoted in a recent study (1260 K, from Glikman et al. 2006). The difference does not seem to arise from technical parts of the fitting method. When we measure the $T_{\mathrm{HD}}$ using a composite spectrum of Glikman et al. (2006) with our method, we find the result to be identical to that of the previous study (i.e., $1260 \mathrm{~K}$ ). Also, we estimate $T_{\mathrm{HD}}$ values of our sample using the methods of Glikman et al. (2006), and the measured $T_{\mathrm{HD}}$ values used in this way are consistent with our results.

The right panel of Figure 16 shows the hot dust luminosities versus the $L_{\mathrm{bol}}$ values for the 19 AGNs. For the bolometric luminosity, we use $L_{5100}$ from our SED fitting multiplied by a bolometric correction factor of 10.3 (Richards et al. 2006). In the figure, we also indicate lines with three different values for the covering factor of the hot dust component $\left(\mathrm{CF}_{\mathrm{HD}}\right)$. Here, the covering factor is given by the ratio of the luminosity from
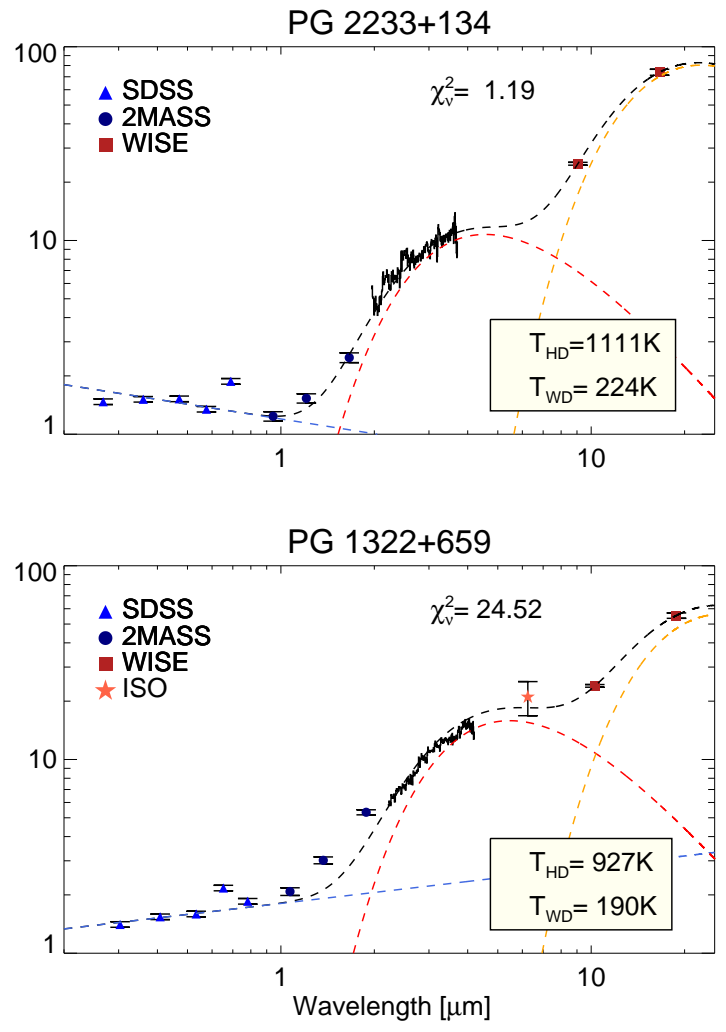

the hot dust component to $L_{\mathrm{bol}}\left(\mathrm{CF}_{\mathrm{HD}}=L_{\mathrm{HD}} / L_{\mathrm{bol}}\right.$; e.g., Maiolino et al. 2007). We find that $L_{\mathrm{HD}}$ correlates well with $L_{\mathrm{bol}}$, and their mean covering factor is found to be 0.38. The measured mean covering factor is similar to several previous results (Sanders et al. 1989; Elvis et al. 1994; Roseboom et al. 2013). However, we note that there is no significant downturn in $\mathrm{CF}_{\mathrm{HD}}$ with $L_{\mathrm{bol}}$, in contrast to previous results which reported that $\mathrm{CF}_{\mathrm{HD}}$ has an anti-correlation with $L_{\text {bol }}$ at the high luminosity end of $10^{46.5} \mathrm{erg} \mathrm{s}^{-1}$ (Wang et al. 2005; Maiolino et al. 2007; Treister et al. 2008; Mor \& Trakhtenbrot 2011; Mor \& Netzer 2012). This discrepancy is quite possibly due to the low number statistics in our data, considering that the anti-correlation found by other groups has a large scatter.

\section{SUMMARY}

Using the $A K A R I$ IRC in grism mode, we obtained $2.5-5.0 \mu \mathrm{m}$ spectra of 83 nearby $(0.002<\mathrm{z}<0.48)$, bright $(K<14$ mag) type-1 AGNs. The Brackett lines are identified in 11 AGNs in the sample, allowing us to derive $M_{\mathrm{BH}}$ estimators that are based on $\operatorname{Br} \alpha$ and $\operatorname{Br} \beta$ line properties. We find the $\operatorname{Br} \beta$-based $M_{\mathrm{BH}}$ estimator using the measured line width and luminosity so as to be useful with an intrinsic scatter of $\sim 0.1$ dex or less with respect to the $M_{\mathrm{BH}}$ values from the reverberation mapping method. On the other hand, the $\operatorname{Br} \alpha$-based estimator shows a larger scatter and no strong dependence on $\sigma_{\mathrm{Br} \alpha}$, demonstrating the difficulty of $\sigma_{\mathrm{Br} \alpha}$ measurements and the need for future data to refine the estimator. However, both $M_{\mathrm{BH}}$ estimators can potentially be applied to derive $M_{\mathrm{BH}}$ values of dusty AGNs due to the low extinction in the Brackett line wavelengths. 

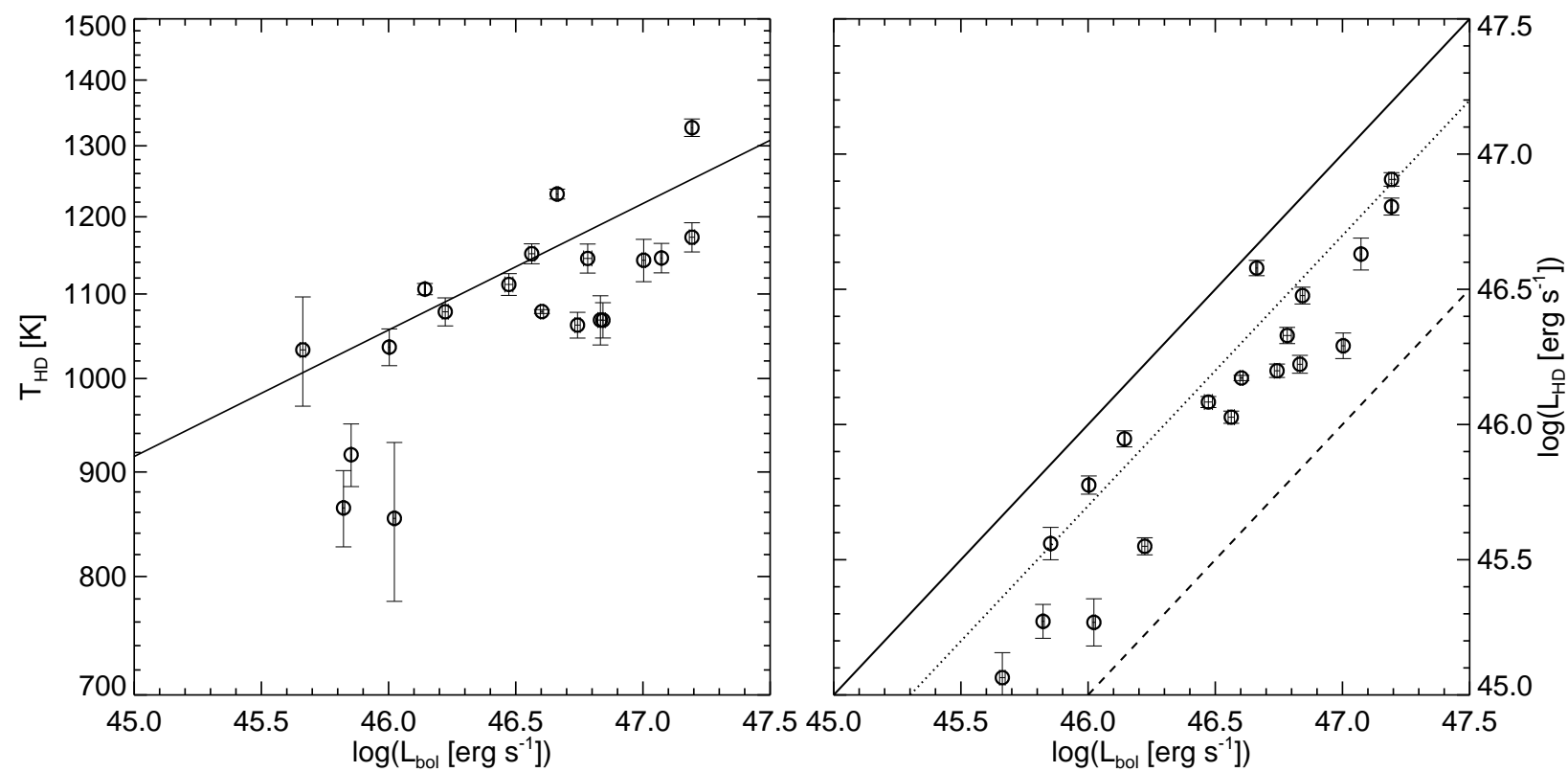

Figure 16. Left: $L_{\mathrm{bol}}$ vs. $T_{\mathrm{HD}}$ of the 19 AGNs. The solid line indicates the best-fit correlation. Right: comparison between $L_{\mathrm{bol}}$ and $L_{\mathrm{HD}}$. The solid, dotted, and dashed lines denote $\mathrm{CF}_{\mathrm{HD}}=1.0,0.5$, and 0.1 , respectively, and the measured mean $\mathrm{CF}_{\mathrm{HD}}$ of the $19 \mathrm{AGNs}$ is 0.38

Table 5

Spectrum of Mrk 335

\begin{tabular}{ccc}
\hline \hline $\begin{array}{c}\lambda \\
{[\mu \mathrm{m}]}\end{array}$ & $\begin{array}{c}f_{\lambda} \\
{\left[\mathrm{erg} \mathrm{s}^{-1} \mathrm{~cm}^{-2} \mu \mathrm{m}^{-1}\right]}\end{array}$ & $\begin{array}{c}f_{\lambda} \text { Uncertainty } \\
{\left[\mathrm{erg} \mathrm{s}^{-1} \mathrm{~cm}^{-2} \mu \mathrm{m}^{-1}\right]}\end{array}$ \\
\hline 2.543 & $3.500 \mathrm{E}-11$ & $4.653 \mathrm{E}-13$ \\
2.552 & $3.363 \mathrm{E}-11$ & $4.595 \mathrm{E}-13$ \\
2.562 & $3.207 \mathrm{E}-11$ & $3.482 \mathrm{E}-13$ \\
2.572 & $3.133 \mathrm{E}-11$ & $2.153 \mathrm{E}-13$ \\
2.581 & $3.106 \mathrm{E}-11$ & $3.536 \mathrm{E}-13$ \\
2.591 & $3.118 \mathrm{E}-11$ & $5.891 \mathrm{E}-13$ \\
2.601 & $3.128 \mathrm{E}-11$ & $2.988 \mathrm{E}-13$
\end{tabular}

\footnotetext{
Note. - This table display only a part of spectrum of Mrk 335. The entire spectra of 83 AGNs and the composite spectrum of 48 PG QSOs are available as a tar file in the electronic version of Astrophysical Journal Supplement Series.
}

The continuum shape is well traced for all of the objects, and we derived the temperatures and luminosities of the hot and warm dust components of 19 luminous AGNs $\left(L_{\text {bol }}>10^{45.7} \mathrm{erg} \mathrm{s}^{-1}\right)$ for which host galaxy contamination is expected to be small $(<10 \%)$. The use of the $A K A R I$ spectra provides the advantage of tracing the NIR continuum densely, thus improving the dust temperature measurements. Our results show the hot dust temperature to be about $1100 \mathrm{~K}$, which is lower than the commonly cited value of $1500 \mathrm{~K}$ but broadly consistent with most recent hot dust temperature measurements. The covering factor of the hot dust component is also derived with a mean $\mathrm{CF}_{\mathrm{HD}}$ of 0.38 , consistent with previous measurements. The AKARI spectral atlas and the tabulated spectral measurements can be downloaded from this article, revealing the rarely studied spectral range of 2.5-5.0 $\mu \mathrm{m}$ for low-redshift AGNs.

This work was supported by the Creative Initiative
Program of the National Research Foundation of Korea (NRF), No. 2008-0060544, funded by the Korea government (MSIP). D.K. acknowledges the fellowship support from the grant NRF-2014-Fostering Core Leaders of Future Program No. 2014-009728 funded by the Korean government. This work was also supported by grant No. 2012R1A4A1028713 (M.G.L. and H.M.L.), grant No. 2012R1A2A2A01006087 (J.H.W.), from the National Research Foundation of Korea (NRF) grant funded by the Korea Government (MSIP). This research is based on observations with $A K A R I$, a JAXA project with the participation of ESA. We thank the anonymous referee for useful comments.

\section{APPENDIX}

We provide reduced 2.5-5.0 $\mu \mathrm{m}$ spectra of 83 AGNs and the composite spectrum of $48 \mathrm{PG}$ QSOs. Table 5 is an example spectrum of Mrk 335. The full version of the reduced spectra is available in machine readable table form. 


\section{REFERENCES}

Barvainis, R. 1987, ApJ, 320, 537

Bentz, M. C., Walsh, J. L., Barth, A. J., et al. 2009, ApJ, 705, 199

Bentz, M. C., Denney, K. D., Grier, C. J., et al. 2013, ApJ, 767, 149

Bruzual, G., \& Charlot, S. 2003, MNRAS, 344, 1000

Cox, A. N. 2000, Allen's Astrophysical Quantities,

Davidson, K., \& Netzer, H. 1979, Reviews of Modern Physics, 51, 715

Denney, K. D., Peterson, B. M., Pogge, R. W., et al. 2010, ApJ, 721,715

Deo, R. P., Richards, G. T., Crenshaw, D. M., \& Kraemer, S. B. 2009, ApJ, 705, 14

Dibai, E. A. 1977, Soviet Astronomy Letters, 3, 1

Elvis, M., Wilkes, B. J., McDowell, J. C., et al. 1994, ApJS, 95, 1

Ferland, G. J., \& Persson, S. E. 1989, ApJ, 347, 656

Ferland, G. J., Korista, K. T., Verner, D. A., et al. 1998, PASP, 110,761

Glikman, E., Helfand, D. J., \& White, R. L. 2006, ApJ, 640, 579

Glikman, E., Helfand, D. J., White, R. L., et al. 2007, ApJ, 667, 673

Green, R. F., Schmidt, M., \& Liebert, J. 1986, ApJS, 61, 305

Greene, J. E., \& Ho, L. C. 2005, ApJ, 630, 122

Greene, J. E., \& Ho, L. C. 2007, ApJ, 670, 92

Grier, C. J., Peterson, B. M., Bentz, M. C., et al. 2008, ApJ, 688, 837

Haas, M., Klaas, U., Müller, S. A. H., et al. 2003, A\&A, 402, 87

Hao, H., Elvis, M., Bongiorno, A., et al. 2012, arXiv:1210.3044

Hopkins, P. F., Hernquist, L., Cox, T. J., et al. 2005, ApJ, 630, 705

Im, M., Griffiths, R. E., \& Ratnatunga, K. U. 1997, ApJ, 475, 457

Im, M., Lee, I., Cho, Y., et al. 2007, ApJ, 664, 64

Im, M., 2010, IAU Symposium, 267, 40

Imanishi, M. 2002, ApJ, 569, 44

Imanishi, M., Nakagawa, T., Shirahata, M., Ohyama, Y., \& Onaka, T. 2010, ApJ, 721, 1233

Imanishi, M., Ichikawa, K., Takeuchi, T., et al. 2011, PASJ, 63, 447

Jiang, L., Fan, X., Brandt, W. N., et al. 2010, Nature, 464, 380

Jun, H. D., \& Im, M. 2013, ApJ, 779, 104

Kaspi, S., Smith, P. S., Netzer, H., et al. 2000, ApJ, 533, 631

Kim, D., Im, M., \& Kim, M. 2010, ApJ, 724, 386

Kim, J. H., Im, M., Lee, H. M., et al. 2012, ApJ, 760, 120

Kobayashi, Y., Sato, S., Yamashita, T., Shiba, H., \& Takami, H. 1993, ApJ, 404, 94

Landt, H., Bentz, M. C., Ward, M. J., et al. 2008, ApJS, 174, 282

Landt, H., Elvis, M., Ward, M. J., et al. 2011, MNRAS, 414, 218

Landt, H., Ward, M. J., Peterson, B. M., et al. 2013, MNRAS, 432,113

Lee, I., Im, M., Kim, M., et al. 2008, ApJS, 175, 116

Li, Y., Hernquist, L., Robertson, B., et al. 2007, ApJ, 665, 187

Maiolino, R., Shemmer, O., Imanishi, M., et al. 2007, A\&A, 468, 979
Markwardt, C. B. 2009, Astronomical Data Analysis Software and Systems XVIII, 411, 251

Marziani, P., Sulentic, J. W., Zamanov, R., et al. 2003, ApJS, 145,199

McCall, M. L. 2004, AJ, 128, 2144

McLure, R. J., \& Dunlop, J. S. 2004, MNRAS, 352, 1390

Minezaki, T., Yoshii, Y., Kobayashi, Y., et al. 2004, ApJ, 600, L35

Mor, R., Netzer, H., \& Elitzur, M. 2009, ApJ, 705, 298

Mor, R., \& Trakhtenbrot, B. 2011, ApJ, 737, L36

Mor, R., \& Netzer, H. 2012, MNRAS, 420, 526

Mouri, H., Kawara, K., Taniguchi, Y., \& Nishida, M. 1990, ApJ, 356, L39

Murakami, H., Baba, H., Barthel, P., et al. 2007, PASJ, 59, 369

Netzer, H., Lutz, D., Schweitzer, M., et al. 2007, ApJ, 666, 806

Ohyama, Y., Onaka, T., Matsuhara, H., et al. 2007, PASJ, 59, 411

Oi, N., Imanishi, M., \& Imase, K. 2010, PASJ, 62, 1509

Onaka, T., Matsuhara, H., Wada, T., et al. 2007, PASJ, 59, 401

Park, D., Kelly, B. C., Woo, J.-H., \& Treu, T. 2012, ApJS, 203, 6

Peterson, B. M., Ferrarese, L., Gilbert, K. M., et al. 2004, ApJ, 613,682

Rees, M. J., Netzer, H., \& Ferland, G. J. 1989, ApJ, 347, 640

Richards, G. T., Lacy, M., Storrie-Lombardi, L. J., et al. 2006 , ApJS, 166, 470

Roseboom, I. G., Lawrence, A., Elvis, M., et al. 2013, MNRAS, 429, 1494

Ruff, A. J., Floyd, D. J. E., Webster, R. L., Korista, K. T., \& Landt, H. 2012, ApJ, 754, 18

Sanders, D. B., Phinney, E. S., Neugebauer, G., Soifer, B. T., \& Matthews, K. 1989, ApJ, 347, 29

Schlafly, E. F., \& Finkbeiner, D. P. 2011, ApJ, 737, 103

Shen, Y., Richards, G. T., Strauss, M. A., et al. 2011, ApJS, 194, 45

Spoon, H. W. W., Armus, L., Cami, J., et al. 2004, ApJS, 154, 184

Suganuma, M., Yoshii, Y., Kobayashi, Y., et al. 2006, ApJ, 639, 46

Tokunaga, A. T., Sellgren, K., Smith, R. G., et al. 1991, ApJ, 380,452

Treister, E., Krolik, J. H., \& Dullemond, C. 2008, ApJ, 679, 140

Urrutia, T., Becker, R. H., White, R. L., et al. 2009, ApJ, 698, 1095

van Dokkum, P. G. 2001, PASP, 113, 1420

Vestergaard, M. 2002, ApJ, 571, 733

Vestergaard, M., \& Peterson, B. M. 2006, ApJ, 641, 689

Wang, J.-M., Zhang, E.-P., \& Luo, B. 2005, ApJ, 627, L5

Williams, M. J., Bureau, M., \& Cappellari, M. 2010, MNRAS, 409,1330

Woo, J.-H., \& Urry, C. M. 2002, ApJ, 579, 530

Woo, J.-H., Kim, J. H., Imanishi, M., \& Park, D. 2012, AJ, 143, 49

Woo, J.-H., Schulze, A., Park, D., et al. 2013, ApJ, 772, 49

Yamada, R., Oyabu, S., Kaneda, H., et al. 2013, arXiv:1307.6356 

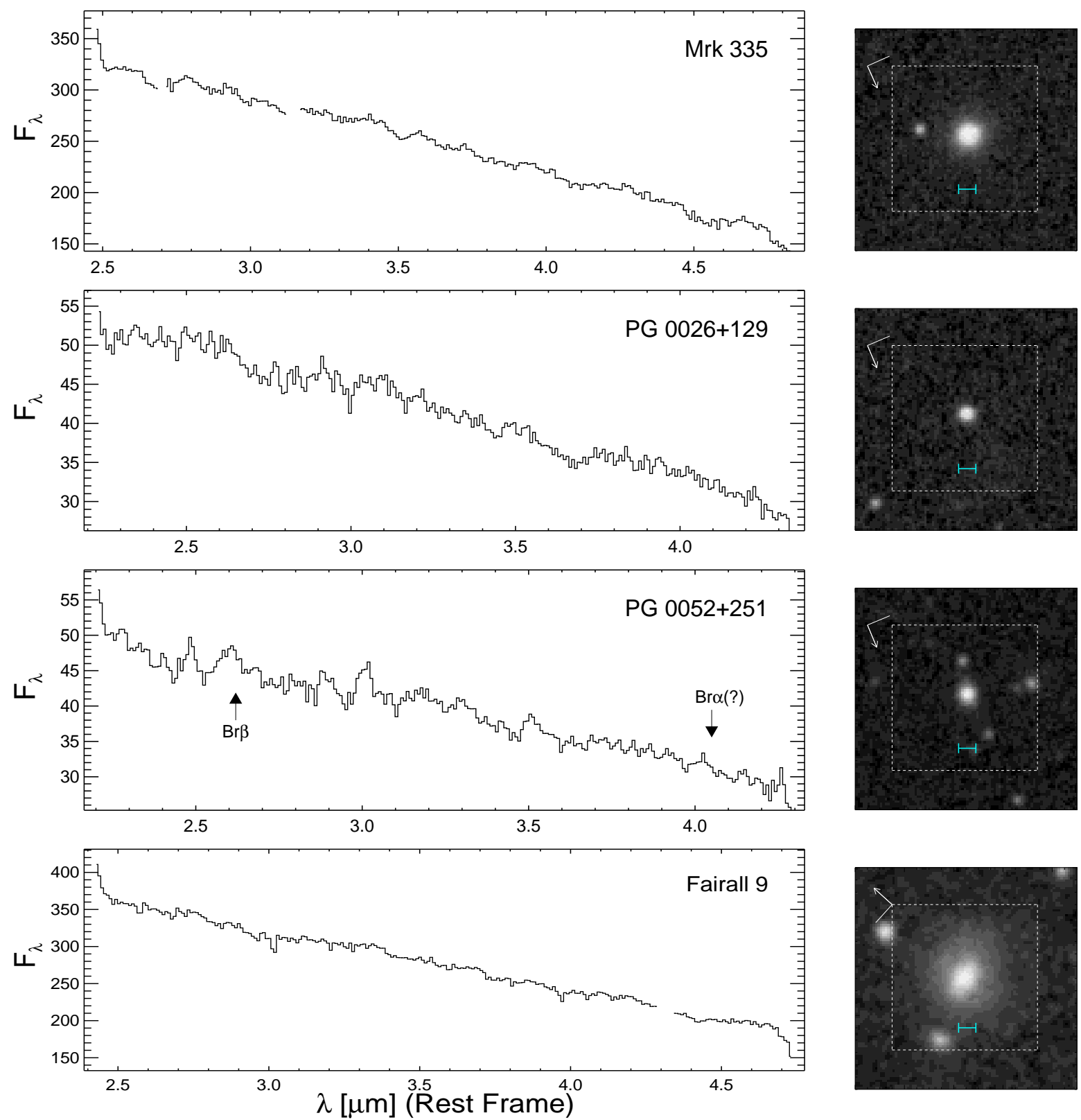

Figure 5. Spectra and images of AGNs in the QSONG sample. The left panels show de-redshifted 2.5-5.0 $\mu$ m spectra and the spectral regions affected by remaining hot or bad pixels are masked out. The ordinate is in units of $10^{-13} \mathrm{erg} \mathrm{s}^{-1} \mathrm{~cm}^{-2} \mu \mathrm{m}^{-1}$. Several hydrogen lines $(\mathrm{P} \alpha, \mathrm{Br} \beta$, and $\mathrm{Br} \alpha)$ and molecular lines ( $\mathrm{PAH}, \mathrm{CO}_{2}$, and $\left.\mathrm{CO}\right)$ are marked on the spectra. When the line detection is not obvious, the line is marked with a question mark. The right panels indicate object images from the digitized sky survey (DSS). The white dashed line box and the cyan solid bar indicate $1.0 \times 1.0$ slit window and $7^{\prime \prime} .3$ width of extraction aperture, respectively. The arrow at the top left denotes north. 

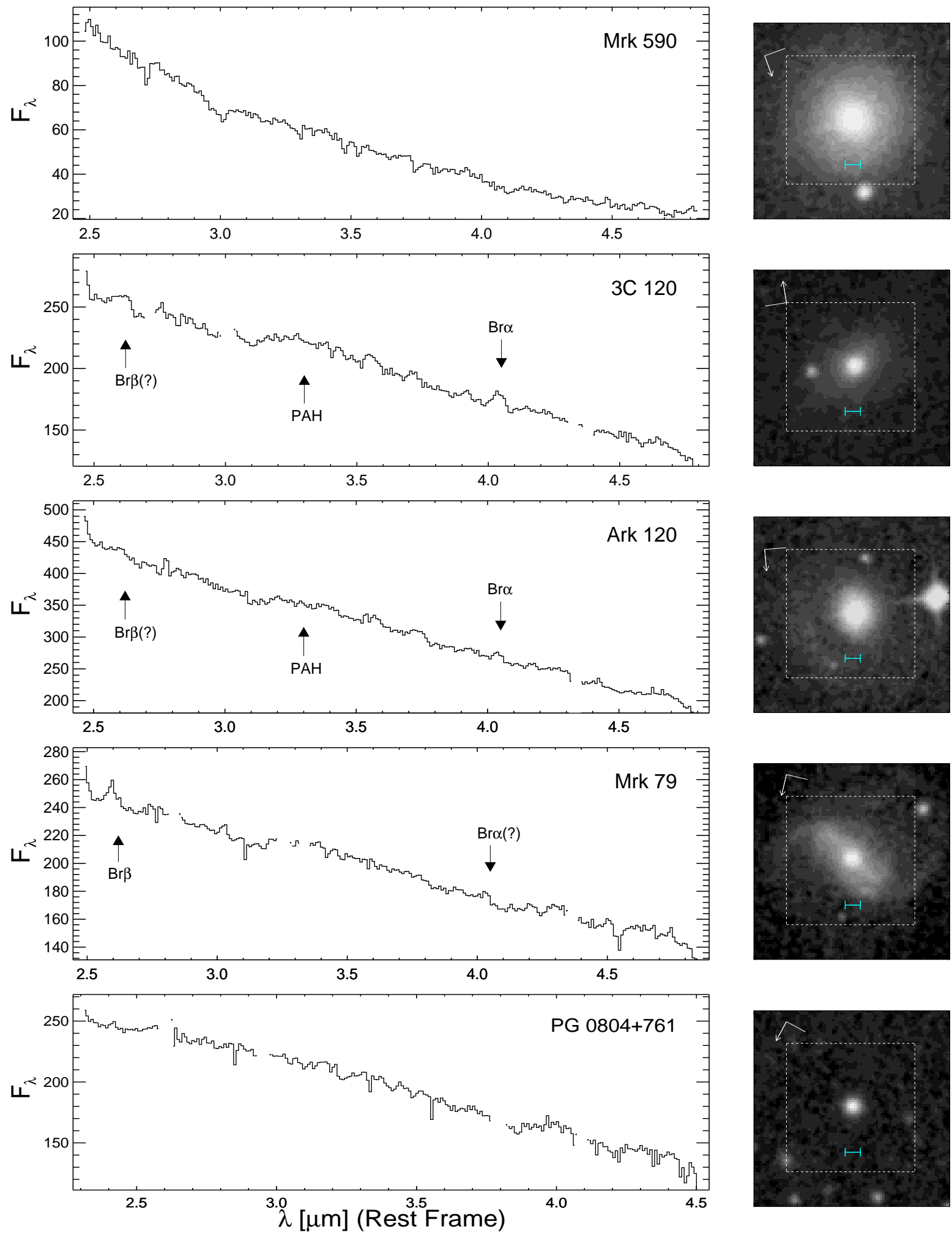

Figure 5. Continued 
Kim et al.
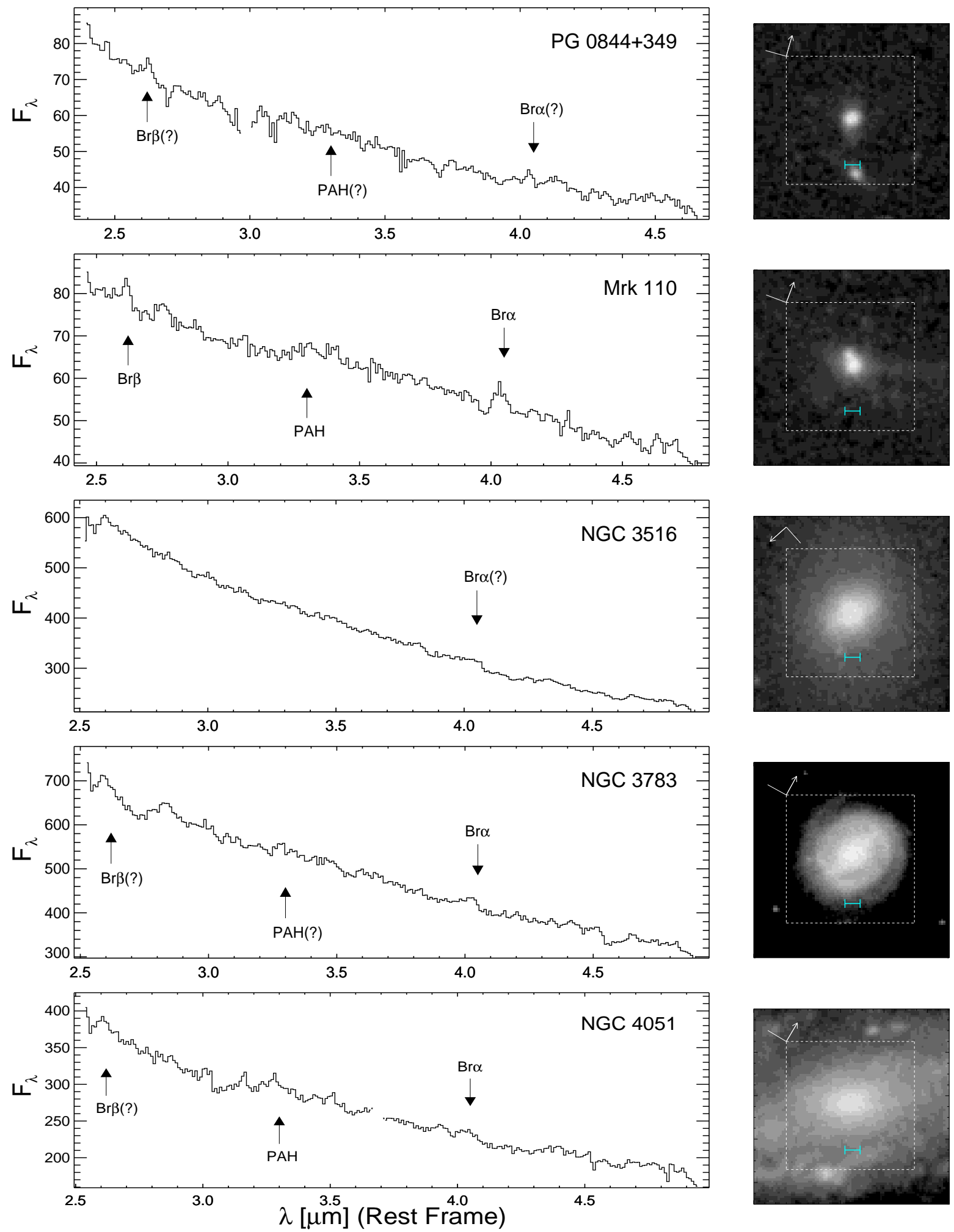

Figure 5. Continued 

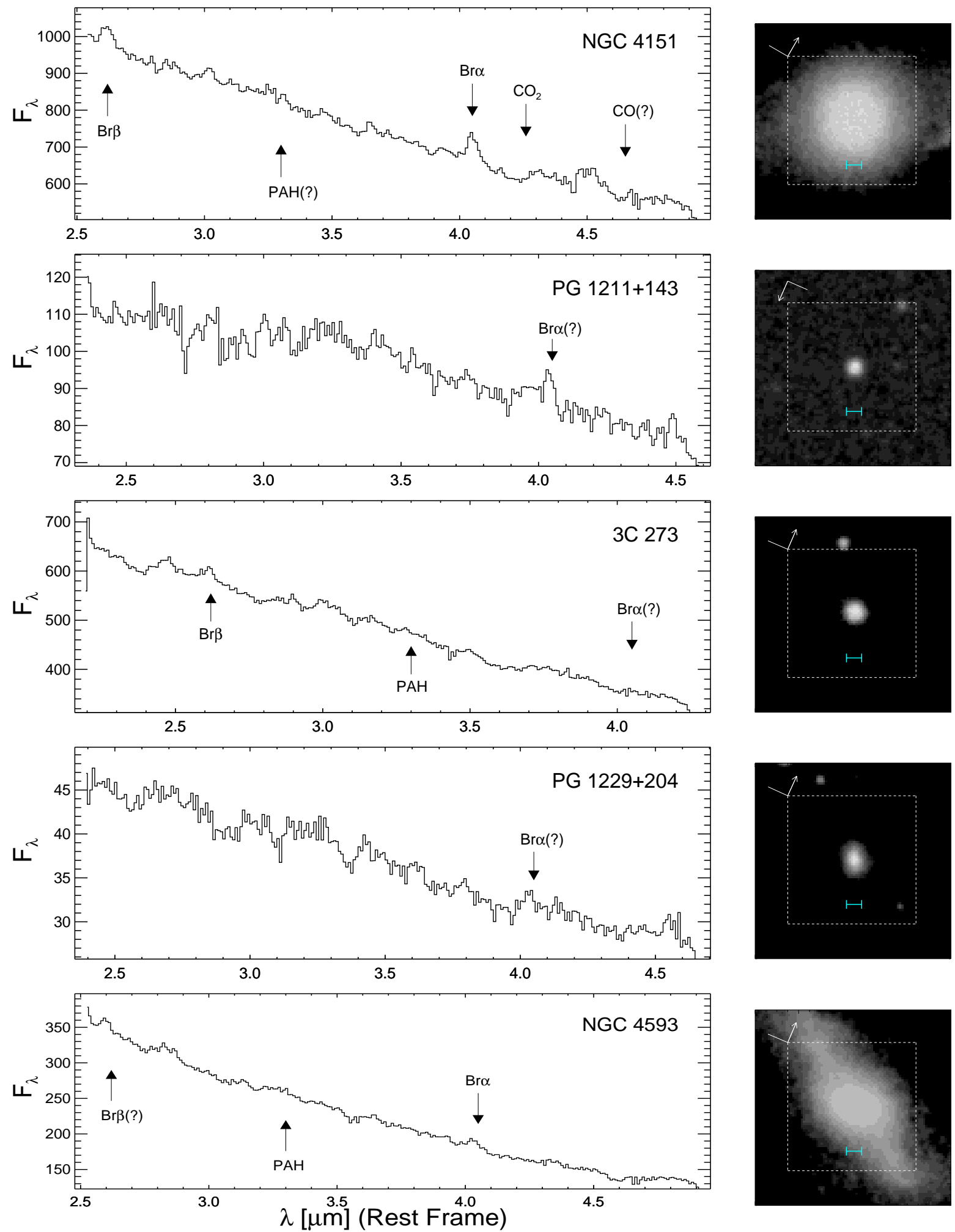

Figure 5. Continued 
Kim et al.
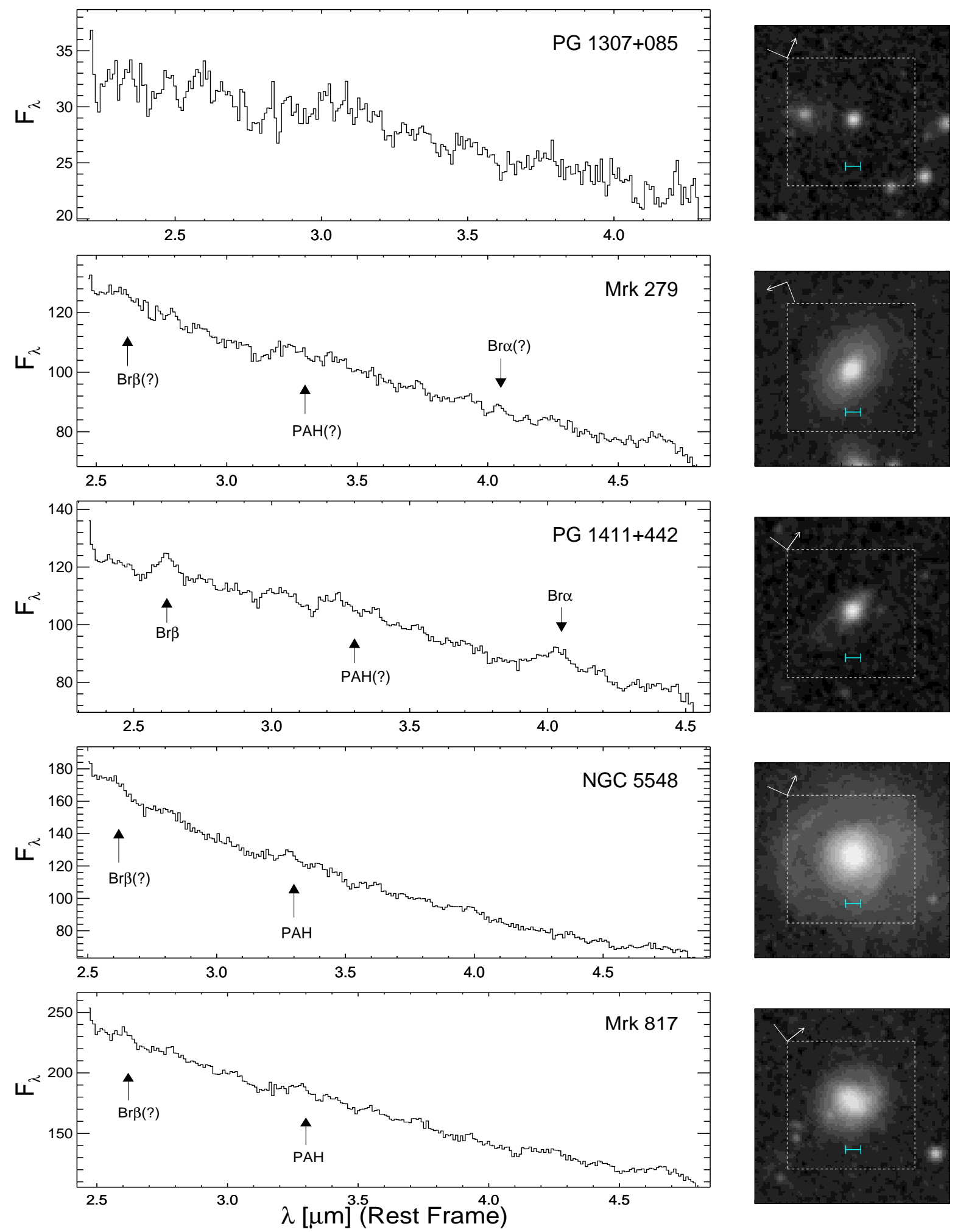

Figure 5. Continued 

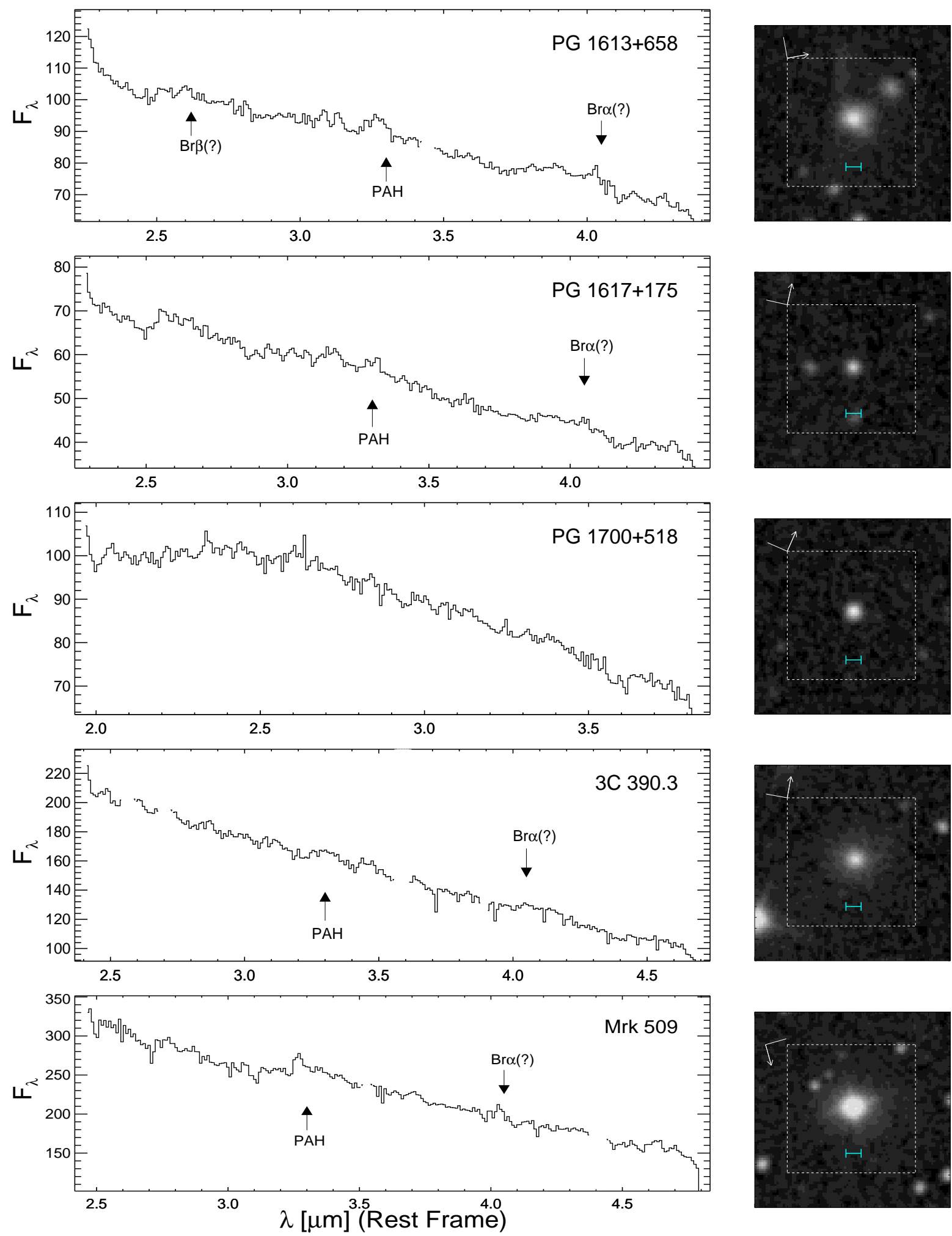

Figure 5. Continued 
Kim et al.
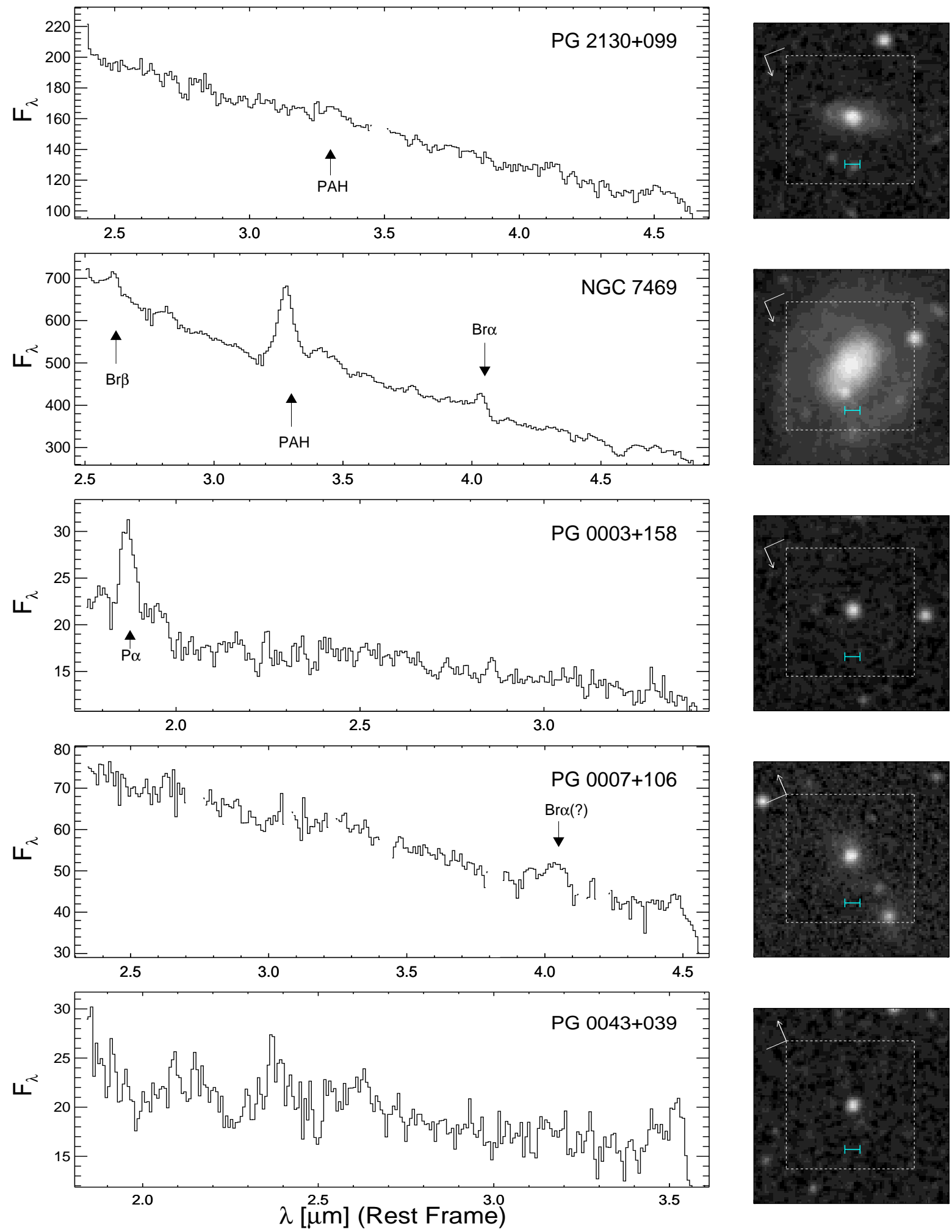

Figure 5. Continued 

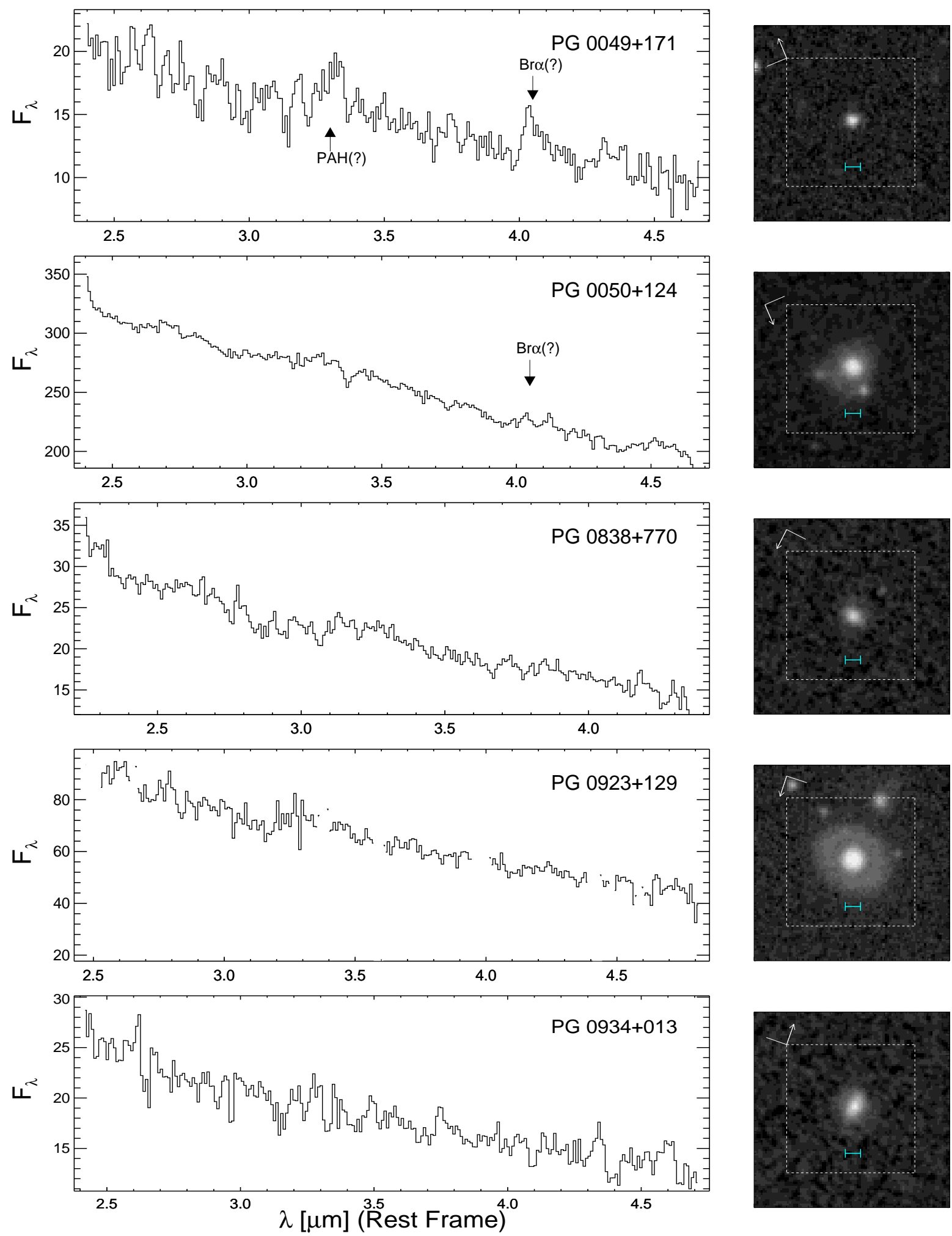

Figure 5. Continued 

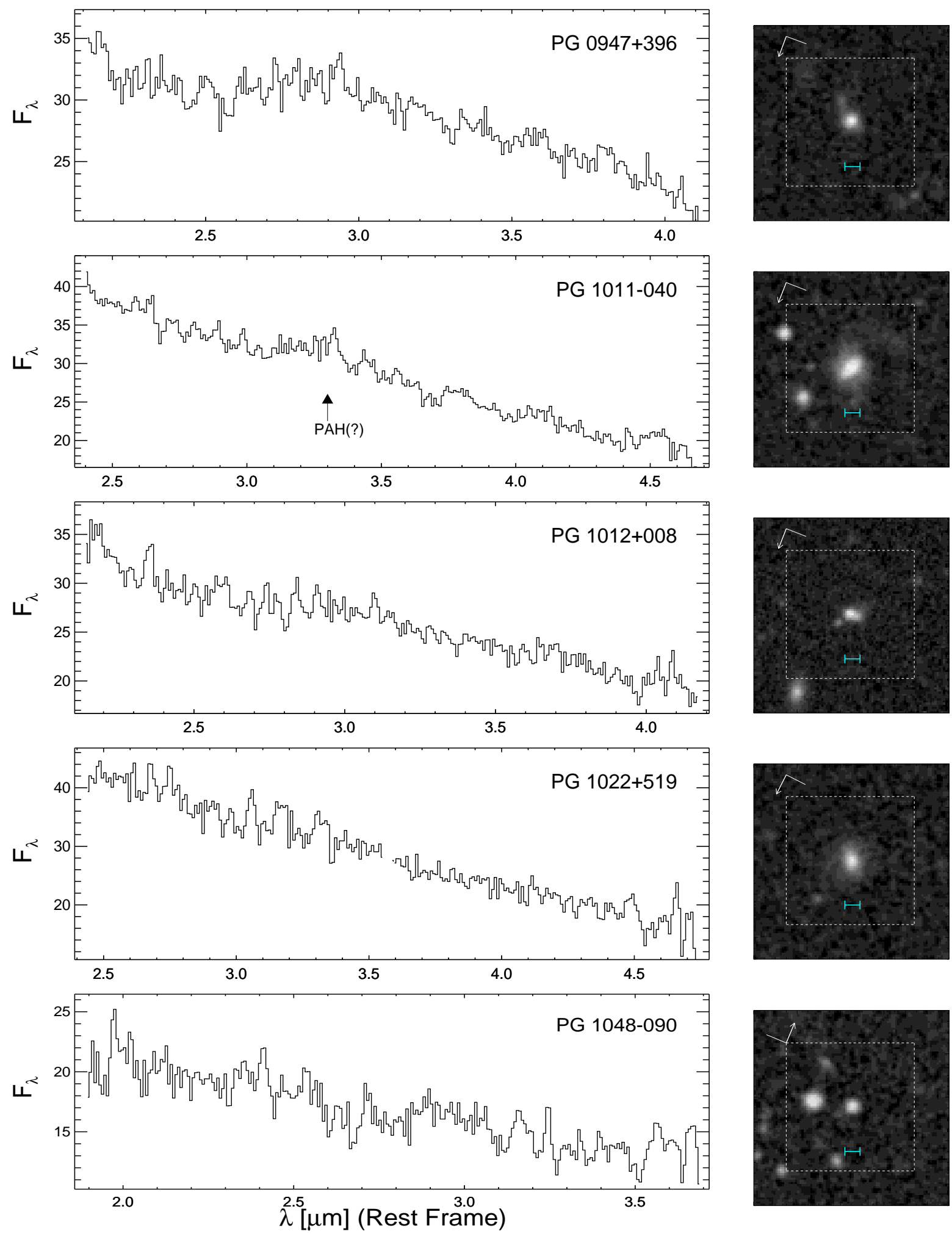

Figure 5. Continued 

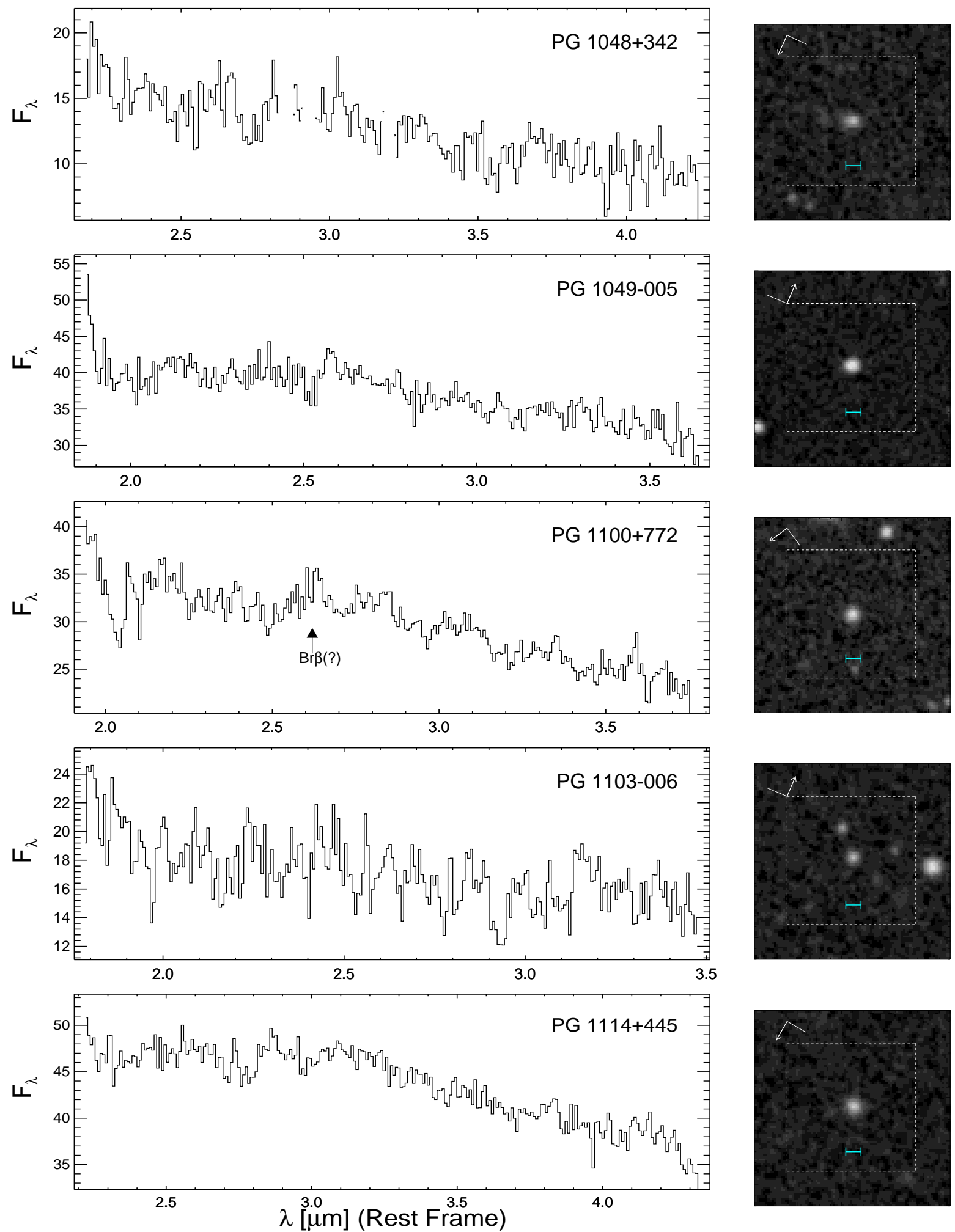

Figure 5. Continued 
Kim et al.
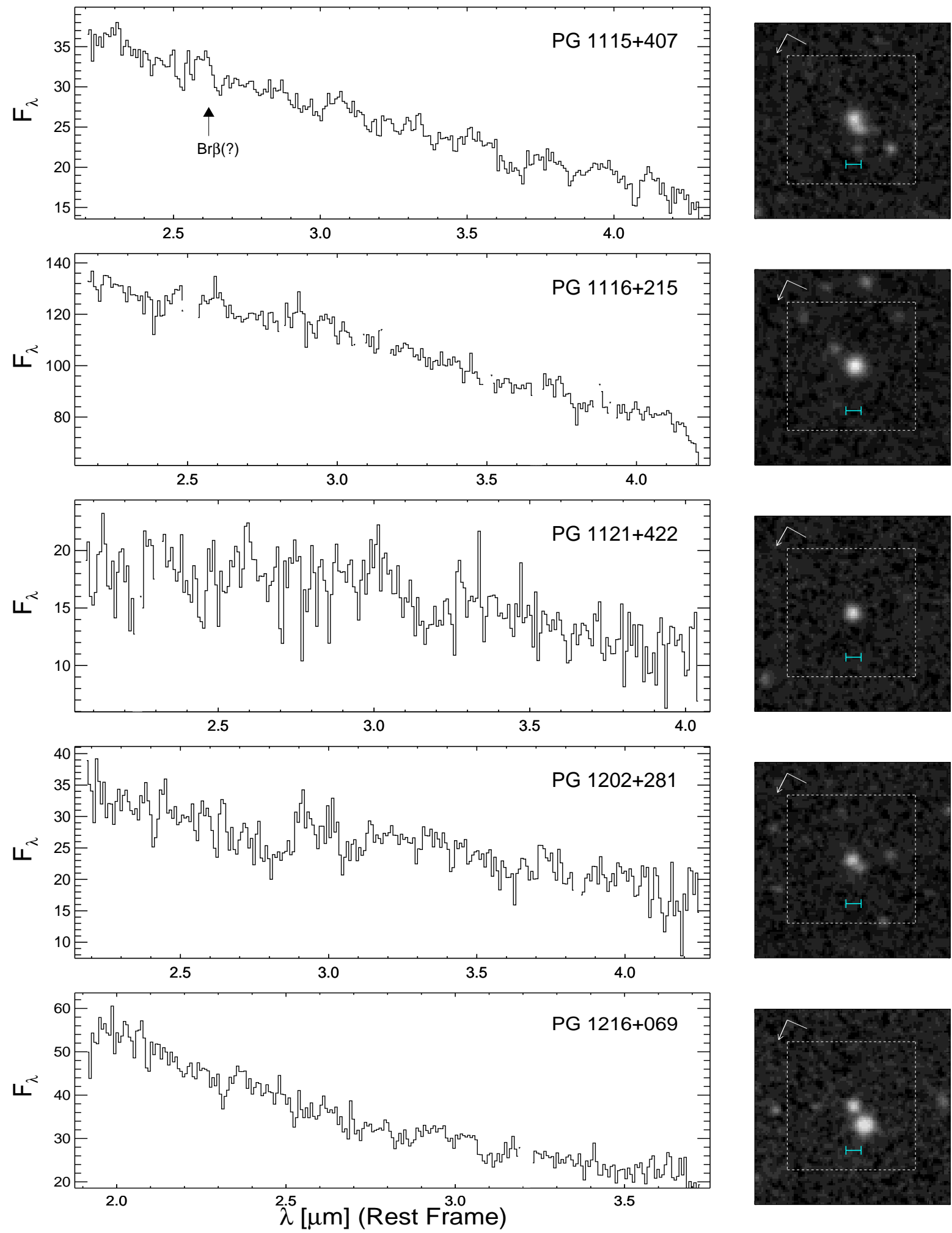

Figure 5. Continued 

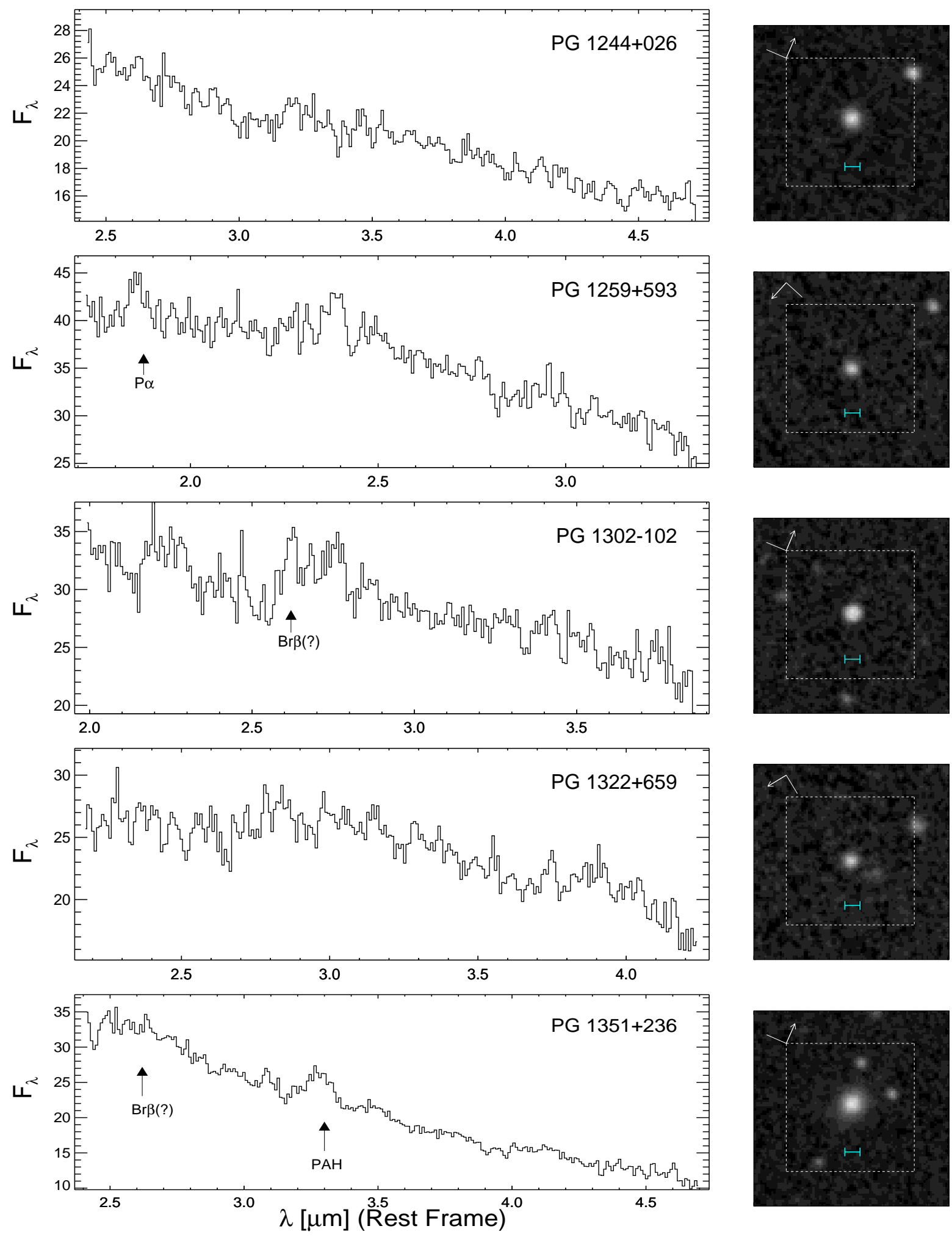

Figure 5. Continued 

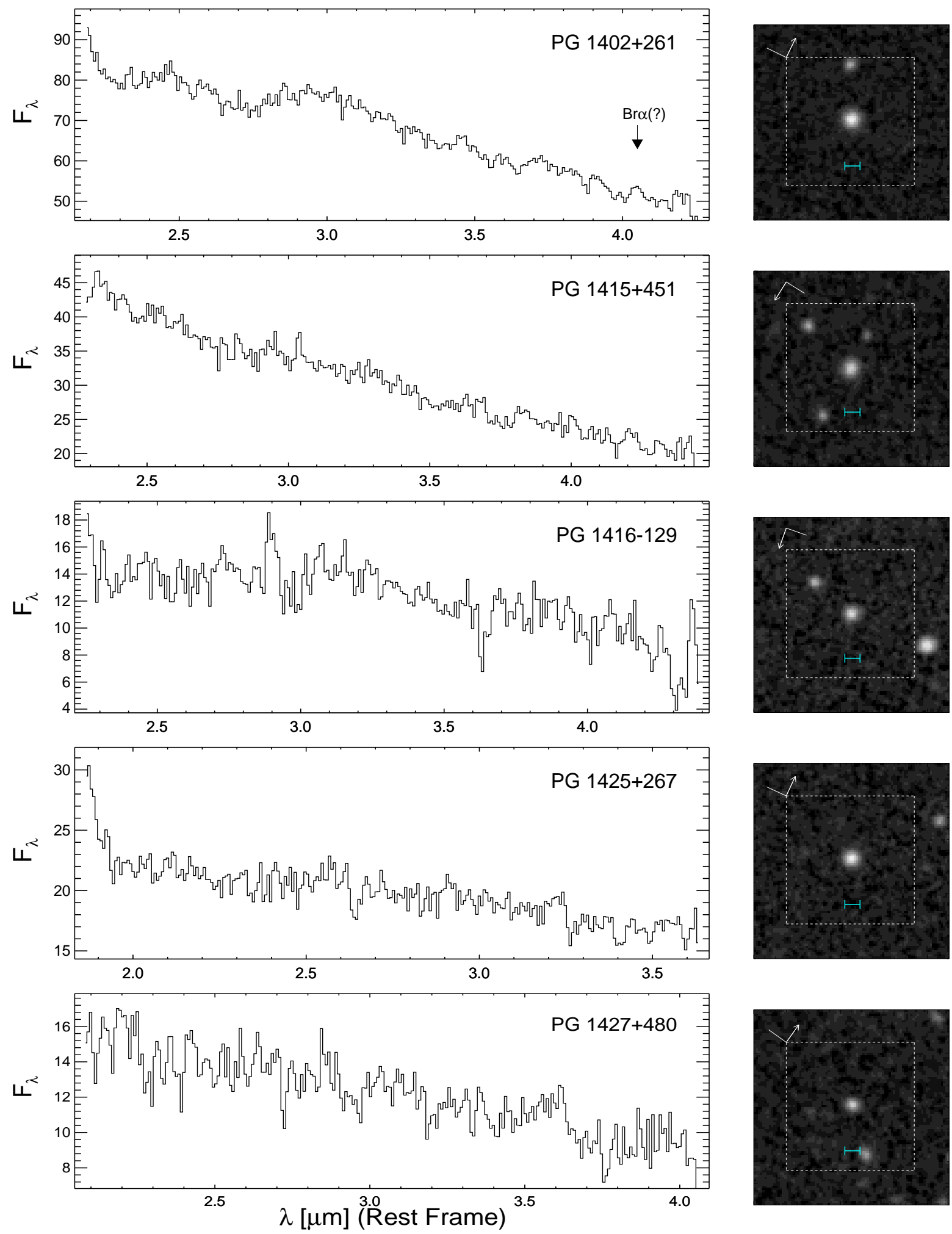

Figure 5. Continued 

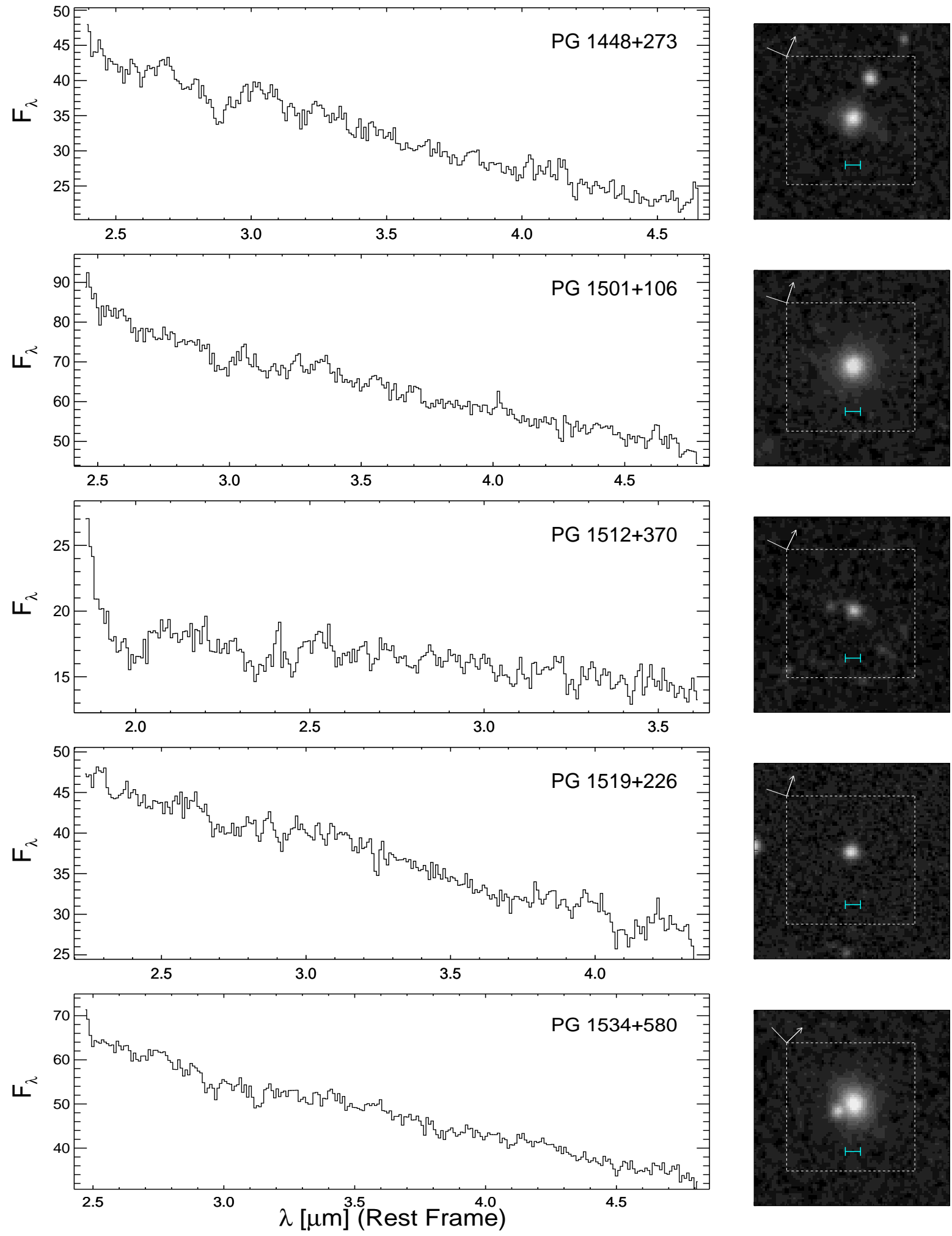

Figure 5. Continued 
Kim et al.
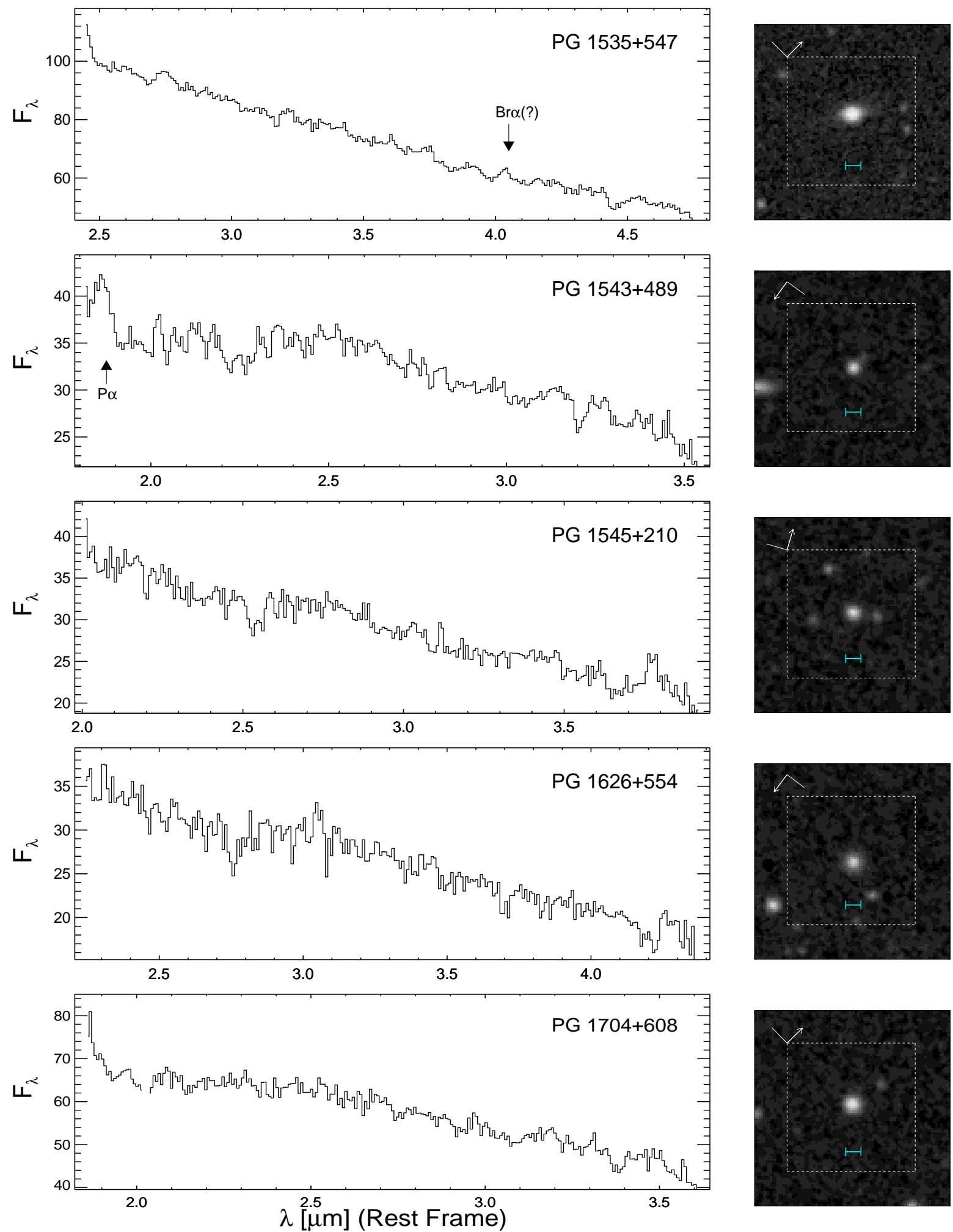

Figure 5. Continued 

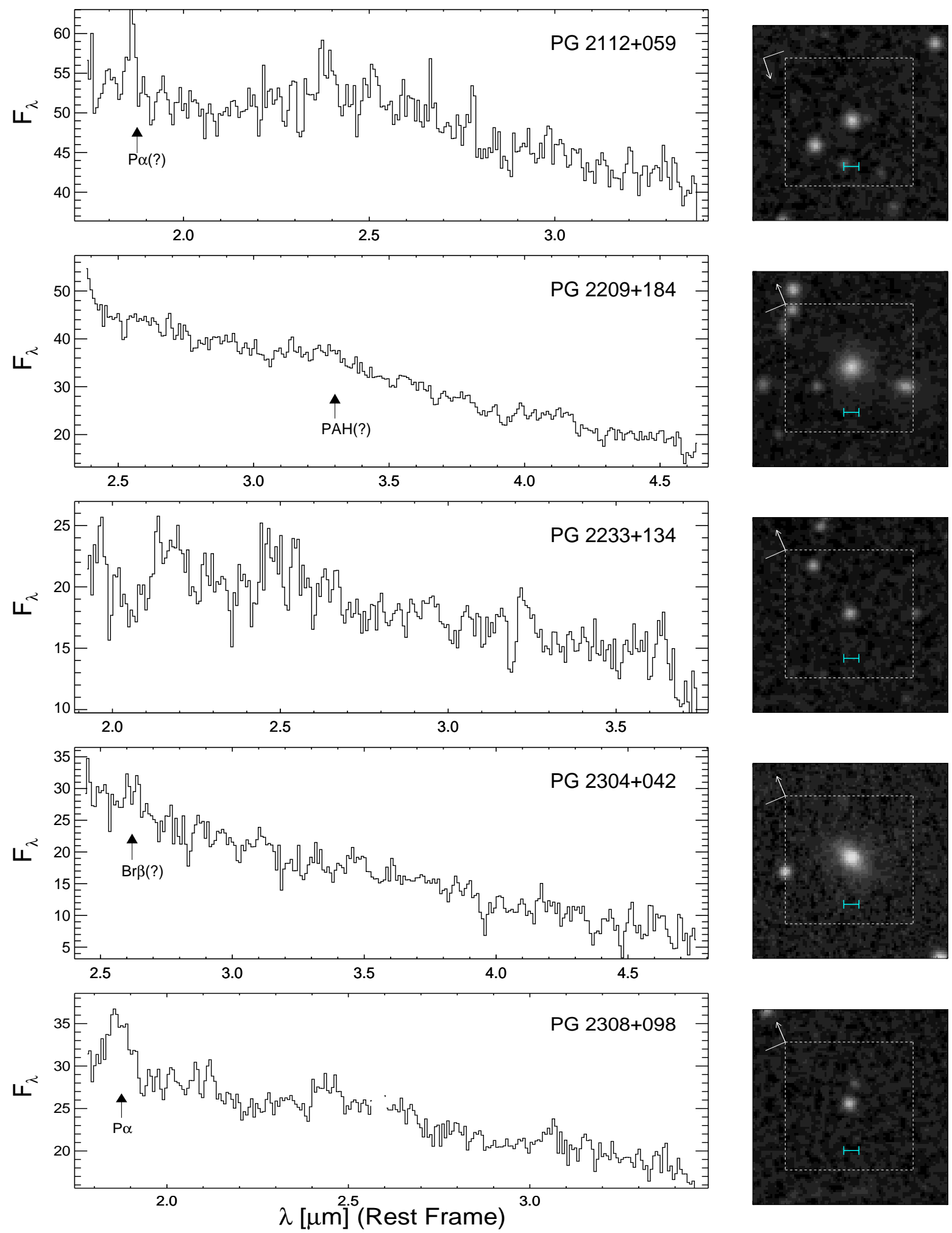

Figure 5. Continued 

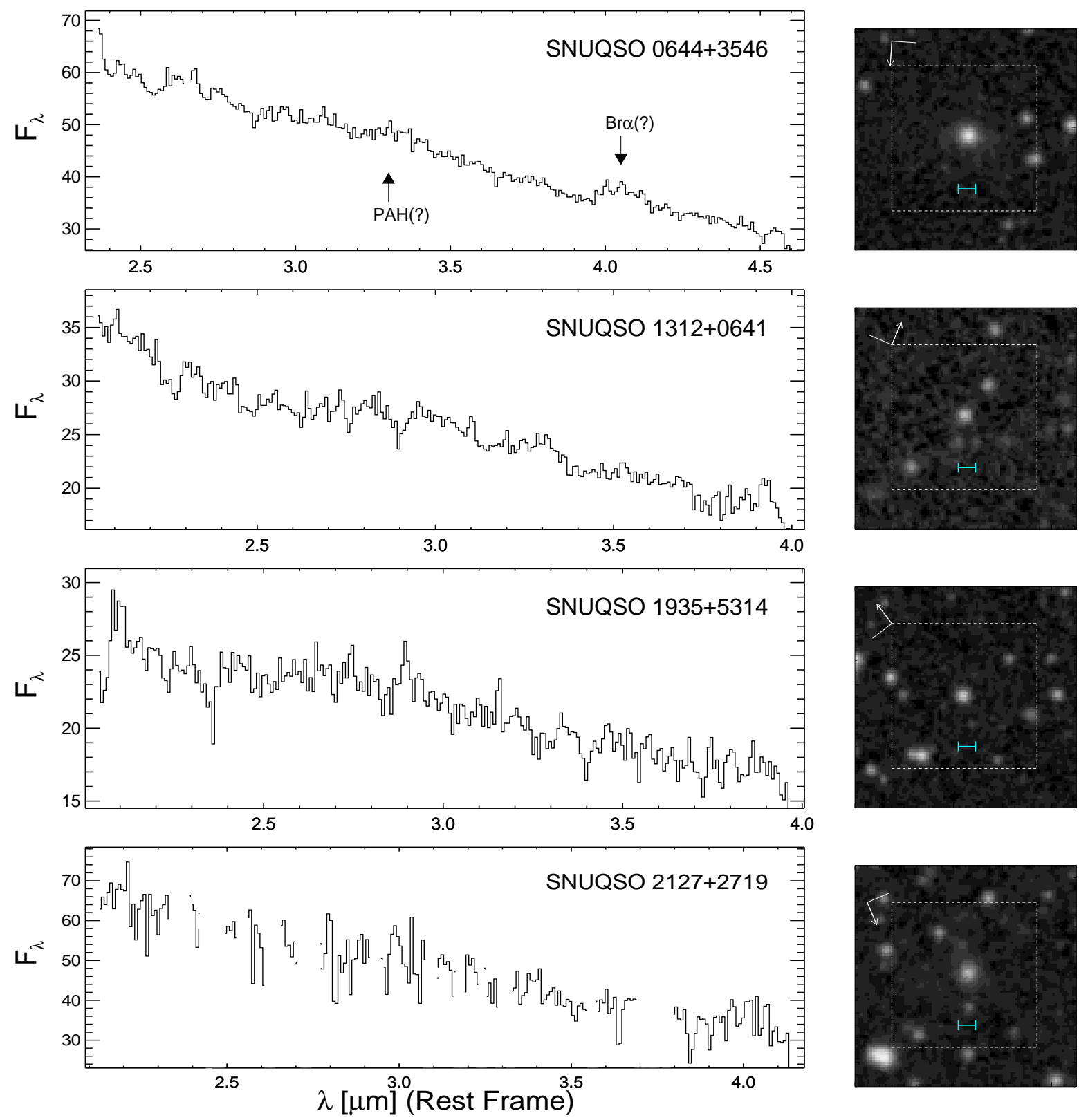

Figure 5. Continued 
Table 1

Object List

\begin{tabular}{|c|c|c|c|c|c|c|c|}
\hline Objects & $\begin{array}{c}\alpha \\
(\mathrm{J} 2000.0)\end{array}$ & $\begin{array}{c}\delta \\
(\mathrm{J} 2000.0)\end{array}$ & $\begin{array}{c}\text { Redshift } \\
z\end{array}$ & $\begin{array}{c}K \\
(\mathrm{mag})\end{array}$ & $\begin{array}{c}\log \left(M_{\mathrm{BH}}\right) \\
\left(M_{\odot}\right) \\
\end{array}$ & $\begin{array}{c}\text { Exposure Time } \\
(\mathrm{s})\end{array}$ & Observed Dates \\
\hline Mrk 335 & 000619.5 & +201210 & 0.025 & 10.59 & $7.11^{\mathrm{a}}$ & 1188 & $2008-06-30$ \\
\hline PG 0026+129 & 002913.6 & +131603 & 0.142 & 12.11 & $8.56^{\mathrm{a}}$ & 1100 & $2008-07-02,03$ \\
\hline PG $0052+251$ & 005452.1 & +252538 & 0.155 & 12.23 & $8.53^{\mathrm{a}}$ & 1144 & $2008-07-13,14$ \\
\hline Fairall 9 & 012345.7 & -584820 & 0.047 & 11.11 & $8.37^{\mathrm{a}}$ & 1188 & $2008-12-01,02$ \\
\hline Mrk 590 & 021433.5 & -004600 & 0.026 & 10.57 & $7.64^{\mathrm{a}}$ & 660 & $2009-07-24$ \\
\hline 3C 120 & 043311.0 & +052115 & 0.033 & 10.57 & $7.71^{\mathrm{a}}$ & 1188 & $2009-02-25$ \\
\hline Ark 120 & $05 \quad 16 \quad 11.4$ & -000859 & 0.032 & 10.22 & $8.14^{\mathrm{a}}$ & 1056 & $2008-09-09$ \\
\hline Mrk 79 & $07 \quad 4232.7$ & +494834 & 0.022 & 10.68 & $7.68^{\mathrm{a}}$ & 1188 & $2008-10-10$ \\
\hline PG 0804+761 & 081058.6 & +760242 & 0.100 & 10.91 & $8.80^{\mathrm{a}}$ & 1188 & $2008-10-04$ \\
\hline PG 0844+349 & 084742.4 & +344504 & 0.064 & 12.01 & $7.93^{\mathrm{a}}$ & 1188 & $2009-04-24$ \\
\hline Mrk 110 & 092512.8 & +521710 & 0.035 & 12.25 & $7.36^{\mathrm{a}}$ & 1056 & $2008-10-29,2009-04-25$ \\
\hline NGC 3516 & 110647.4 & +723406 & 0.008 & 9.61 & $7.46^{\mathrm{b}}$ & 1188 & $2008-10-25,26$ \\
\hline NGC 3783 & 113901.7 & -374418 & 0.009 & 10.01 & $7.44^{\mathrm{a}}$ & 1188 & $2008-07-03$ \\
\hline NGC 4051 & 120309.6 & +443152 & 0.002 & 10.01 & $6.20^{\mathrm{b}}$ & 748 & $2009-05-29,30$ \\
\hline NGC 4151 & 121032.5 & +392420 & 0.003 & 8.51 & $7.09^{\mathrm{a}}$ & 1056 & $2008-06-03,04$ \\
\hline PG $1211+143$ & 121417.7 & +140312 & 0.080 & 11.29 & $8.13^{\mathrm{a}}$ & 1144 & $2009-12-18,19$ \\
\hline $3 \mathrm{C} 273$ & 122906.7 & +020308 & 0.158 & 9.97 & $8.91^{\mathrm{a}}$ & 1848 & $2008-06-26,27,12-27$ \\
\hline PG $1229+204$ & 123203.6 & +200929 & 0.063 & 12.14 & $7.83^{\mathrm{a}}$ & 1188 & $2009-06-20$ \\
\hline NGC 4593 & 123939.4 & -052039 & 0.009 & 10.03 & $6.69^{\mathrm{a}}$ & 1144 & $2008-07-02,2009-01-01$ \\
\hline PG $1307+085$ & 130947.0 & +08 1948 & 0.155 & 12.42 & $8.61^{\mathrm{a}}$ & 1144 & $2008-07-03,04$ \\
\hline Mrk 279 & 135303.4 & +691829 & 0.030 & 10.39 & $7.51^{\mathrm{a}}$ & 1144 & $2008-11-14,15,16$ \\
\hline PG $1411+442$ & 141348.3 & +440014 & 0.089 & 11.50 & $8.61^{\mathrm{a}}$ & 1144 & $2008-06-28,29,30$ \\
\hline NGC 5548 & 141759.5 & +250812 & 0.017 & 10.13 & $7.61^{\mathrm{b}}$ & 1144 & $2008-07-13,14$ \\
\hline Mrk 817 & 143622.0 & +584739 & 0.031 & 10.90 & $7.60^{\mathrm{a}}$ & 660 & $2008-06-12$ \\
\hline PG $1613+658$ & 161357.1 & +654309 & 0.129 & 11.41 & $8.41^{\mathrm{a}}$ & 1144 & $2008-06-07,08$ \\
\hline PG $1617+175$ & 162011.2 & +172427 & 0.112 & 12.41 & $8.74^{\mathrm{a}}$ & 1188 & $2008-08-21$ \\
\hline PG $1700+518$ & 170124.8 & +514920 & 0.292 & 11.98 & $8.85^{\mathrm{a}}$ & 924 & $2008-08-15,17$ \\
\hline 3C 390.3 & 184208.9 & +794617 & 0.056 & 11.71 & $8.42^{\mathrm{a}}$ & 1188 & $2008-09-10$ \\
\hline Mrk 509 & 204409.7 & -104324 & 0.034 & 10.19 & $8.12^{\mathrm{a}}$ & 1012 & $2009-04-30$ \\
\hline PG 2130+099 & 213227.8 & +100819 & 0.062 & 10.66 & $7.54^{\mathrm{c}}$ & 1100 & $2009-05-20$ \\
\hline NGC 7469 & $\begin{array}{lll}23 & 03 & 15.6\end{array}$ & +085226 & 0.016 & 9.762 & $7.05^{\mathrm{a}}$ & 1100 & $2008-06-10,11$ \\
\hline PG $0003+158$ & 000559.2 & +160949 & 0.450 & 13.53 & $9.24^{\mathrm{d}}$ & 1144 & $2008-06-30,12-29,30$ \\
\hline PG $0007+106$ & 001031.0 & +105830 & 0.089 & 11.78 & $8.69^{\mathrm{d}}$ & 1188 & $2009-12-28$ \\
\hline PG $0043+039$ & 004547.3 & +041024 & 0.385 & 13.59 & $9.09^{\mathrm{d}}$ & 1012 & $2010-01-02$ \\
\hline PG $0049+171$ & 005154.8 & +172558 & 0.064 & 13.34 & $8.31^{\mathrm{d}}$ & 1188 & $2010-01-09$ \\
\hline PG $0050+124$ & 005334.9 & +124136 & 0.061 & 10.35 & $7.41^{\mathrm{d}}$ & 1144 & $2008-07-08,09$ \\
\hline PG $0838+770$ & 084445.3 & +765310 & 0.131 & 13.15 & $8.12^{\mathrm{d}}$ & 1188 & $2008-10-06$ \\
\hline PG 0923+129 & 092603.3 & +124404 & 0.029 & 11.30 & $8.56^{\mathrm{d}}$ & 396 & $2009-11-12$ \\
\hline PG $0934+013$ & 093701.0 & +010543 & 0.050 & 13.50 & $7.01^{\mathrm{d}}$ & 792 & $2009-05-17$ \\
\hline PG $0947+396$ & 095048.4 & +392651 & 0.205 & 12.76 & $8.64^{\mathrm{d}}$ & 1188 & $2009-11-07$ \\
\hline PG 1011-040 & 101420.7 & -041840 & 0.058 & 12.65 & $7.28^{\mathrm{d}}$ & 1144 & $2009-11-28$ \\
\hline PG $1012+008$ & 101454.9 & +003337 & 0.186 & 13.08 & $8.21^{\mathrm{d}}$ & 1100 & $2009-11-27$ \\
\hline PG $1022+519$ & 102531.3 & +514035 & 0.044 & 12.71 & $7.11^{\mathrm{d}}$ & 396 & $2009-11-07$ \\
\hline PG $1048-090$ & 105129.9 & -09 1810 & 0.344 & 13.95 & $9.17^{\mathrm{d}}$ & 1188 & $2009-06-08$ \\
\hline PG $1048+342$ & 105143.9 & +335927 & 0.167 & 13.60 & $8.33^{\mathrm{d}}$ & 704 & $2009-11-22$ \\
\hline PG 1049-005 & 105151.4 & -005118 & 0.359 & 12.84 & $9.15^{\mathrm{d}}$ & 1144 & $2009-06-04$ \\
\hline PG $1100+772$ & 110413.7 & +765858 & 0.311 & 13.05 & $9.24^{\mathrm{d}}$ & 1188 & $2008-10-18$ \\
\hline PG 1103-006 & 110631.8 & -005252 & 0.423 & 13.85 & $9.29^{\mathrm{d}}$ & 792 & $2009-06-08$ \\
\hline PG $1114+445$ & 111706.4 & +441333 & 0.143 & 12.33 & $8.56^{\mathrm{d}}$ & 1144 & $2009-11-21,22$ \\
\hline PG $1115+407$ & 111830.3 & +402554 & 0.154 & 12.76 & $7.63^{\mathrm{d}}$ & 1144 & $2009-11-24$ \\
\hline PG $1116+215$ & 111908.7 & +211918 & 0.176 & 11.54 & $8.48^{\mathrm{d}}$ & 1056 & $2009-12-03$ \\
\hline PG $1121+422$ & 112439.2 & +420145 & 0.225 & 13.26 & $8.00^{\mathrm{d}}$ & 396 & $2009-11-26$ \\
\hline PG $1202+281$ & 120442.1 & +275412 & 0.165 & 12.87 & $8.58^{\mathrm{d}}$ & 396 & $2009-12-11$ \\
\hline PG $1216+069$ & 121920.9 & +063839 & 0.331 & 13.30 & $9.16^{\mathrm{d}}$ & 616 & $2009-12-24$ \\
\hline PG $1244+026$ & 124635.2 & +022209 & 0.048 & 13.19 & $6.49^{\mathrm{d}}$ & 1100 & $2008-06-30,12-31$ \\
\hline PG $1259+593$ & 130112.9 & +590207 & 0.477 & 13.07 & $8.88^{\mathrm{d}}$ & 1144 & $2009-11-27,28$ \\
\hline PG $1302-102$ & 130533.0 & -103319 & 0.278 & 12.85 & $8.84^{\mathrm{d}}$ & 1100 & $2009-07-10,11$ \\
\hline PG $1322+659$ & 132349.5 & +654148 & 0.168 & 12.85 & $8.25^{\mathrm{d}}$ & 1056 & $2009-11-21,22$ \\
\hline PG $1351+236$ & 135406.4 & +232549 & 0.055 & 13.01 & $8.53^{\mathrm{d}}$ & 792 & $2008-07-09$ \\
\hline PG $1402+261$ & 140516.2 & +255534 & 0.164 & 12.16 & $7.91^{\mathrm{d}}$ & 1144 & $2008-07-09$ \\
\hline PG $1415+451$ & 141700.7 & +445606 & 0.113 & 12.23 & $7.98^{\mathrm{d}}$ & 1144 & $2008-12-29,2009-06-28$ \\
\hline PG $1416-129$ & 141903.8 & -131044 & 0.129 & 13.34 & $9.01^{\mathrm{d}}$ & 1100 & $2010-01-27$ \\
\hline PG $1425+267$ & 142735.6 & +263215 & 0.364 & 13.57 & $9.70^{\mathrm{d}}$ & 1188 & $2008-07-16$ \\
\hline PG $1427+480$ & 142943.1 & +474726 & 0.220 & 13.48 & $8.05^{\mathrm{d}}$ & 1188 & $2009-06-29,07-01$ \\
\hline PG $1448+273$ & 145108.7 & +270927 & 0.065 & 12.05 & $6.94^{\mathrm{d}}$ & 1188 & $2009-07-22,23$ \\
\hline PG $1501+106$ & 150401.2 & +102616 & 0.036 & 11.47 & $8.49^{\mathrm{d}}$ & 1188 & $2009-08-02$ \\
\hline PG $1512+370$ & 151443.0 & +365050 & 0.370 & 13.69 & $9.34^{\mathrm{d}}$ & 1100 & $2008-07-22$ \\
\hline PG $1519+226$ & 152114.2 & +222744 & 0.137 & 12.47 & $7.91^{\mathrm{d}}$ & 1188 & $2009-08-02,03$ \\
\hline PG $1534+580$ & 153552.3 & +575409 & 0.029 & 12.18 & $8.17^{\mathrm{d}}$ & 1100 & $2008-06-29,12-31,2009-01-01$ \\
\hline
\end{tabular}


Table 1 - Continued

\begin{tabular}{cccccccc}
\hline \hline Objects & $\begin{array}{c}\alpha \\
(\mathrm{J} 2000.0)\end{array}$ & $\begin{array}{c}\delta \\
(\mathrm{J} 2000.0)\end{array}$ & $\begin{array}{c}\text { Redshift } \\
z\end{array}$ & $\begin{array}{c}K \\
(\mathrm{mag})\end{array}$ & $\begin{array}{c}\log \left(M_{\mathrm{BH}}\right) \\
\left(M_{\odot}\right)\end{array}$ & $\begin{array}{c}\text { Exposure Time } \\
(\mathrm{s})\end{array}$ & Observed Dates \\
\hline PG 1535+547 & 153638.3 & +543333 & 0.038 & 11.63 & $7.16^{\mathrm{d}}$ & 704 & $2008-07-06,08$ \\
PG 1543+489 & 154530.2 & +484609 & 0.399 & 13.14 & $7.96^{\mathrm{d}}$ & 1188 & $2009-01-17,20,21$ \\
PG 1545+210 & 154743.5 & +205217 & 0.264 & 13.18 & $9.28^{\mathrm{d}}$ & 616 & $2008-08-11$ \\
PG 1626+554 & 162756.1 & +552232 & 0.133 & 12.67 & $8.46^{\mathrm{d}}$ & 1188 & $2010-01-22$ \\
PG 1704+608 & 170441.4 & +604431 & 0.371 & 12.43 & $9.36^{\mathrm{d}}$ & 1100 & $2008-07-23$ \\
PG 2112+059 & 211452.6 & +060742 & 0.466 & 12.85 & $8.97^{\mathrm{d}}$ & 1056 & $2009-05-13,14$ \\
PG 2209+184 & 221153.9 & +184150 & 0.070 & 12.55 & $8.73^{\mathrm{d}}$ & 1144 & $2009-12-03,05$ \\
PG 2233+134 & 223607.7 & +134355 & 0.325 & 13.81 & $8.00^{\mathrm{d}}$ & 1188 & $2009-12-07,08$ \\
PG 2304+042 & 230702.9 & +043257 & 0.042 & 12.54 & $8.53^{\mathrm{d}}$ & 748 & $2009-12-12$ \\
PG 2308+098 & 231117.7 & +100815 & 0.433 & 13.23 & $9.56^{\mathrm{d}}$ & 1012 & $2008-12-14$ \\
SNUQSO 0644+3546 & 064410.8 & +354644 & 0.077 & 11.82 & $8.86^{\mathrm{e}}$ & 1188 & $2008-10-01$ \\
SNUQSO 1312+0641 & 131204.7 & +064107 & 0.239 & 13.20 & $9.09^{\mathrm{e}}$ & 1100 & $2008-07-06,2009-01-03$ \\
SNUQSO 1935+5314 & 193521.1 & +531411 & 0.248 & 13.14 & $8.61^{\mathrm{e}}$ & 1188 & $2008-11-17,2009-05-11$ \\
SNUQSO 2127+2719 & 212756.4 & +271905 & 0.197 & 12.63 & $8.74^{\mathrm{e}}$ & 396 & $2009-05-26$ \\
& & & & & & & \\
\hline
\end{tabular}

${ }^{\mathrm{a}}$ The $M_{\mathrm{BH}}$ values are estimated from reverberation mapping method (Peterson et al. 2004).

b The $M_{\mathrm{BH}}$ values are estimated from reverberation mapping method (Dennev et al. 2010).

c The $M_{\mathrm{BH}}$ values are estimated from reverberation mapping method (Grier et al. 2008).

d The $M_{\mathrm{BH}}$ values are based on $\mathrm{FWHM}_{\mathrm{H} \beta}$ and $L_{5100}$ (Vestergaard \& Peterson 2006).

${ }^{\mathrm{e}}$ The $M_{\mathrm{BH}}$ values are based on $\mathrm{FWHM}_{\mathrm{H} \alpha}$ and $L_{\mathrm{H} \alpha}$ (Lee et al. 2008; Im et al. 2007). 


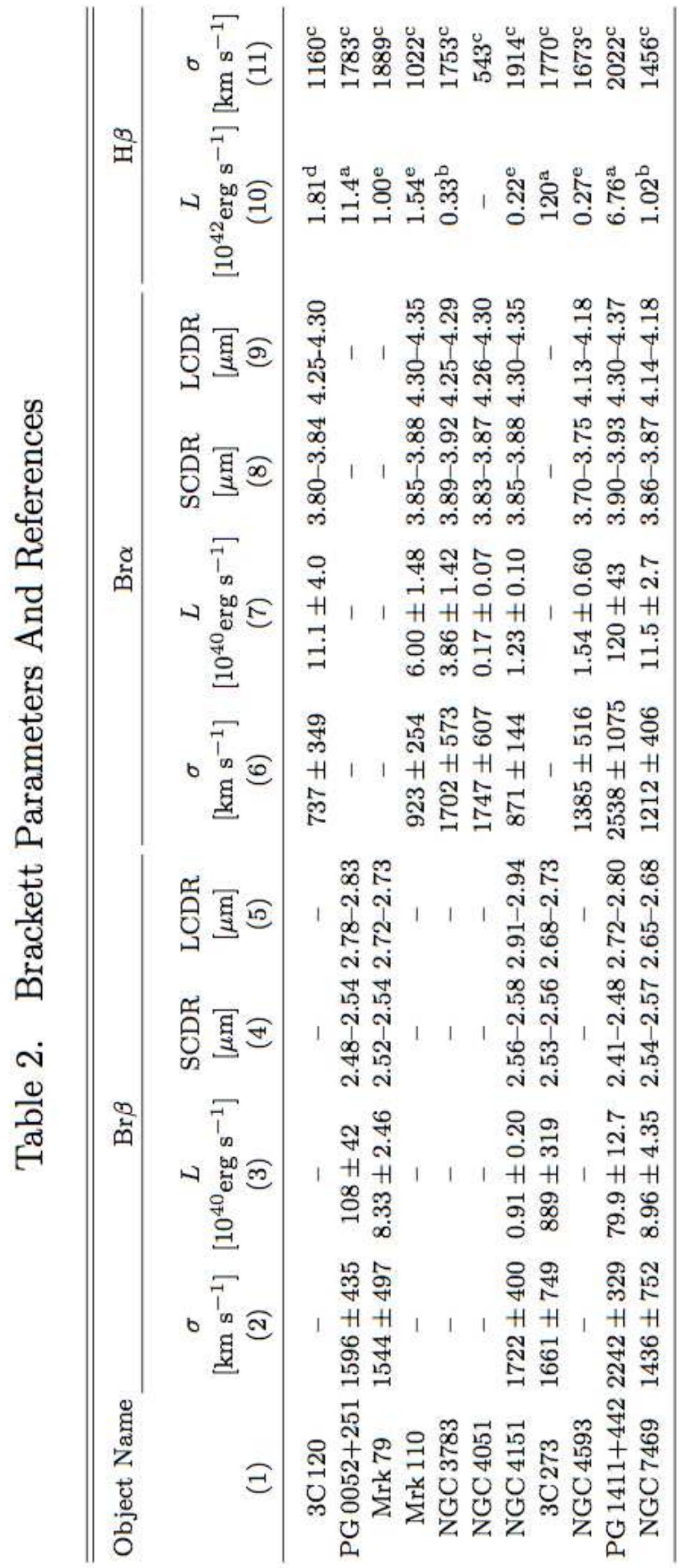

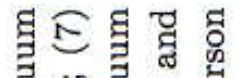

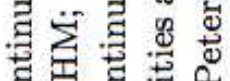

ठํㅇㅇㅇㅇ

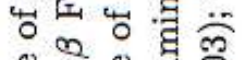

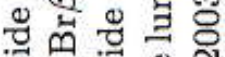

के के ब

응

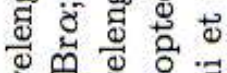

ต

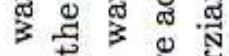

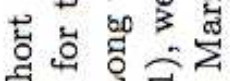

क्न झ。

ङ

३

क्ष

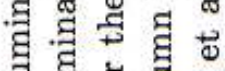

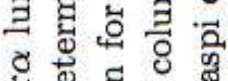

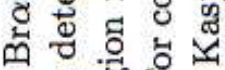

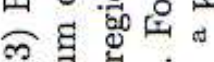

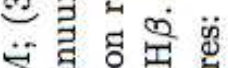

密范范芯

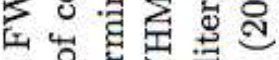

ชั ข

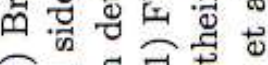

กิ

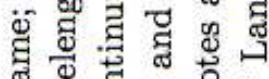

ฮ

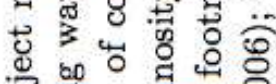

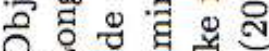

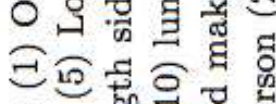

उ ช

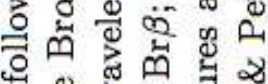

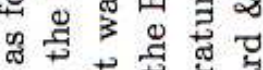

ฮั

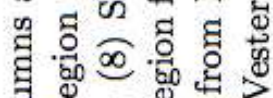

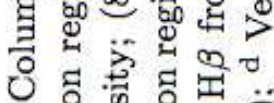

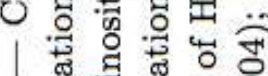

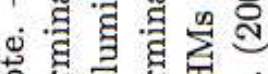

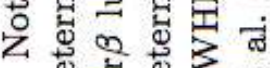

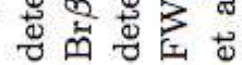

